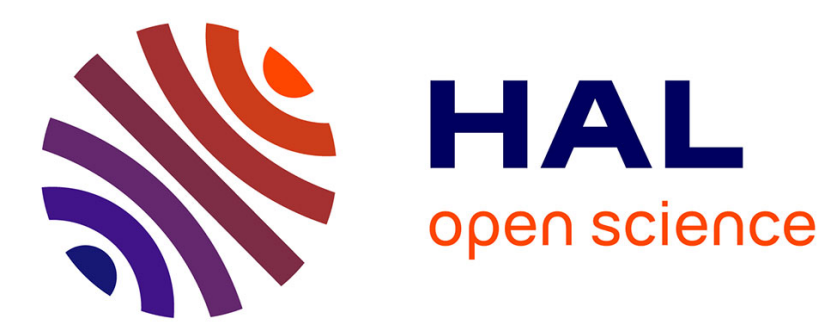

\title{
Évaluation de la courbe d'apprentissage des extractions par spatules de Thierry
}

\author{
Ouidade El Haloui
}

\section{To cite this version:}

Ouidade El Haloui. Évaluation de la courbe d'apprentissage des extractions par spatules de Thierry. Médecine humaine et pathologie. 2013. dumas-01063313

\section{HAL Id: dumas-01063313 https://dumas.ccsd.cnrs.fr/dumas-01063313}

Submitted on 18 Sep 2014

HAL is a multi-disciplinary open access archive for the deposit and dissemination of scientific research documents, whether they are published or not. The documents may come from teaching and research institutions in France or abroad, or from public or private research centers.
L'archive ouverte pluridisciplinaire HAL, est destinée au dépôt et à la diffusion de documents scientifiques de niveau recherche, publiés ou non, émanant des établissements d'enseignement et de recherche français ou étrangers, des laboratoires publics ou privés. 


\section{EL HALOUI OUIDADE}

Interne des hôpitaux

\section{EVALUATION DE LA COURBE D'APPRENTISSAGE DES EXTRACTIONS PAR SPATULES DE THIERRY}

Nice, 2013 



\section{Evaluation de la courbe d'apprentissage des extractions par spatules de Thierry}

*******

\section{THESE}

Présentée et soutenue publiquement devant la Faculté de Médecine Nice

Le 28/06/2013

Par

Ouidade EL HALOUI

Née le 20 septembre 1984 à Saint-Quentin

Interne en Gynécologie Obstétrique

Pour obtenir le grade de

\section{Docteur en Médecine (diplôme d'Etat)}

Examinateur de la thèse :

Monsieur le Professeur André Bongain

Monsieur le Professeur Patrick Baqué

Monsieur le Professeur Jean-Paul Fournier

Monsieur le Docteur Jean Breaud

Monsieur le Docteur Jerôme Delotte
Président

Assesseur

Assesseur

Assesseur

Directeur de thèse 
Liste des professeurs au 1er mars 2013 à la Faculté de Médecine de Nice

Doyen

Assesseurs

Conservateur de la bibliothèque

Chef des services administratifs

Doyens Honoraires

\section{Professeurs Honoraires}

M. BAQUÉ Patrick

M. BOILEAU Pascal M. HEBUTERNE Xavier M. LEVRAUT Jacques

M. SCALABRE Grégory

Mme HIZEBRY Valérie

M. AYRAUD Noël

M. RAMPAL Patrick

\author{
M. BALAS Daniel \\ M. BLAIVE Bruno \\ M. BOQUET Patrice \\ M. BOURGEON André \\ M. BRUNETON Jean-Noël \\ Mme BUSSIERE Françoise \\ M. CHATEL Marcel \\ M. COUSSEMENT Alain \\ M. DARCOURT Guy \\ M. DELMONT Jean \\ M. DEMARD François \\ M. DOLISI Claude \\ M. FREYCHET Pierre \\ M. GILLET Jean-Yves \\ M. GRELLIER Patrick \\ M. HARTER Michel \\ M. INGLESAKIS Jean-André \\ M. LAMBERT Jean-Claude
}

M.C.A. Honoraire

M.C.U. Honoraires

\author{
M. LALANNE Claude-Michel \\ M. LAPALUS Philippe \\ M. LAZDUNSKI Michel \\ M. LEFEBVRE Jean-Claude \\ M. LE BAS Pierre \\ M. LE FICHOUX Yves \\ M. LOUBIERE Robert \\ M. MARIANI Roger \\ M. MASSEYEFF René \\ M. MATTEI Mathieu \\ M. MOUIEL Jean \\ Mme MYQUEL Martine \\ M. OLLIER Amédée \\ M. SCHNEIDER Maurice \\ M. SERRES Jean-Jacques \\ M. TOUBOL Jacques \\ M. TRAN Dinh Khiem \\ M. ZIEGLER Gérard
}

Mlle ALLINE Madeleine

M. ARNOLD Jacques

M. BASTERIS Bernard

Mlle CHICHMANIAN Rose-Marie

M. EMILIOZZI Roméo

M. GASTAUD Marcel

M. GIRARD-PIPAU Fernand

Mme MEMRAN Nadine

M. MENGUAL Raymond

M. POIREE Jean-Claude

Mme ROURE Marie-Claire 


\section{PROFESSEURS CLASSE EXCEPTIONNELLE}

$\begin{array}{ll}\text { M. } & \text { BENCHIMOL Daniel } \\ \text { M. } & \text { CAMOUS Jean-Pierre } \\ \text { M. } & \text { DELLAMONICA Pierre } \\ \text { M. } & \text { DESNUELLE Claude } \\ \text { Mme } & \text { EULLER-ZIEGLER Liana } \\ \text { M. } & \text { FENICHEL Patrick } \\ & \\ \text { M. } & \text { FUZIBET Jean-Gabriel } \\ \text { M. } & \text { FRANCO Alain } \\ \text { M. } & \text { GASTAUD Pierre } \\ \text { M. } & \text { GERARD Jean-Pierre } \\ \text { M. } & \text { GILSON Éric } \\ \text { M. } & \text { GRIMAUD Dominique } \\ \text { M. } & \text { HEBUTERNE Xavier } \\ \text { M. } & \text { HOFMAN Paul } \\ \text { M. } & \text { LACOUR Jean-Philippe } \\ \text { Mme } & \text { LEBRETON Elisabeth } \\ & \\ \text { M. } & \text { ORTONNE Jean-Paul } \\ \text { M. } & \text { PRINGUEY Dominique } \\ \text { M. } & \text { SANTINI Joseph } \\ \text { M. } & \text { THYSS Antoine } \\ \text { M. } & \text { VAN OBBERGHEN Emmanuel }\end{array}$

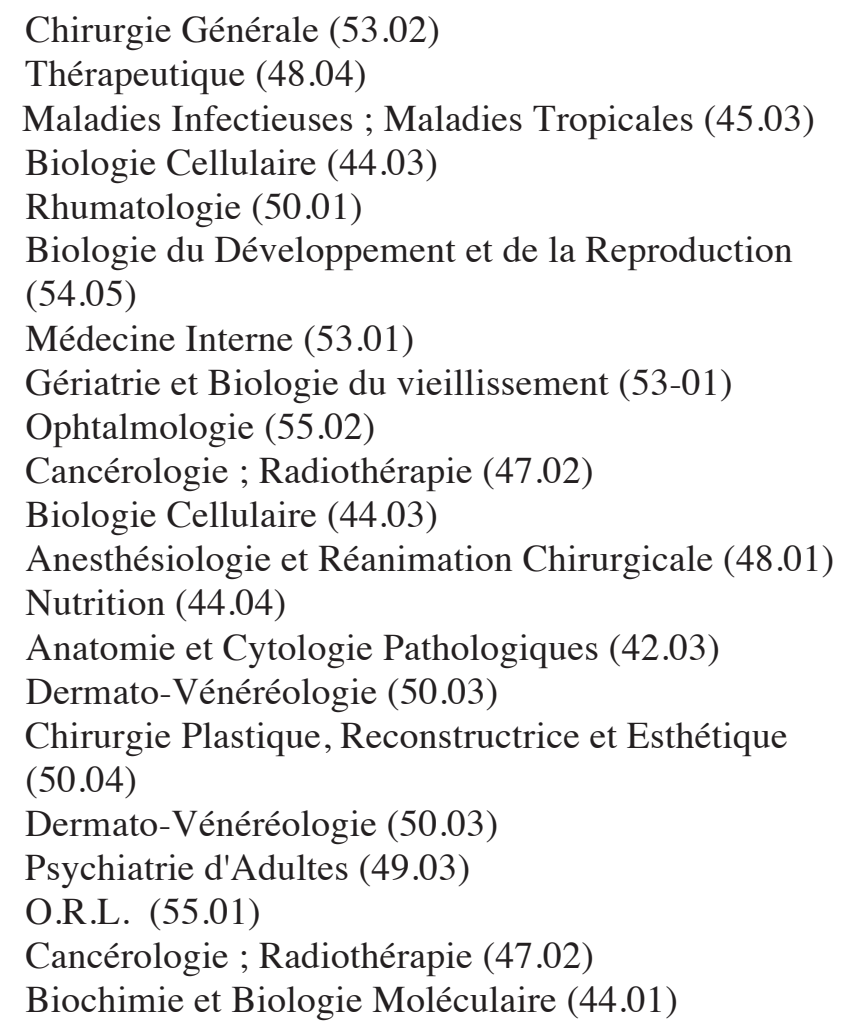

\section{PROFESSEURS PREMIERE CLASSE}

$\begin{array}{ll}\text { M. } & \text { AMIEL Jean } \\ \text { M. } & \text { BATT Michel } \\ \text { M. } & \text { BERARD Etienne } \\ \text { M. } & \text { BERNARDIN Gilles } \\ \text { M. } & \text { BOILEAU Pascal } \\ \text { M. } & \text { BONGAIN André } \\ \text { Mme } & \text { CRENESSE Dominique } \\ \text { M. } & \text { DARCOURT Jacques } \\ \text { M. } & \text { DE PERETTI Fernand } \\ \text { M. } & \text { DRICI Milou-Daniel } \\ \text { M. } & \text { ESNAULT Vincent } \\ \text { M. } & \text { GIBELIN Pierre } \\ \text { M. } & \text { GUGENHEIM Jean } \\ \text { M. } & \text { HASSEN KHODJA Reda } \\ \text { Mme } & \text { ICHAI Carole } \\ \text { M. } & \text { LONJON Michel } \\ \text { M. } & \text { MARQUETTE Charles-Hugo } \\ \text { M. } & \text { MARTY Pierre } \\ \text { M. } & \text { MICHIELS Jean-François } \\ \text { M. } & \text { MOUNIER Nicolas } \\ \text { M. } & \text { MOUROUX Jérôme } \\ \text { M. } & \text { PADOVANI Bernard }\end{array}$

\author{
Urologie (52.04) \\ Chirurgie Vasculaire (51.04) \\ Pédiatrie (54.01) \\ Réanimation Médicale (48.02) \\ Chirurgie Orthopédique et Traumatologique (50.02) \\ Gynécologie-Obstétrique (54.03) \\ Physiologie (44.02) \\ Biophysique et Médecine Nucléaire (43.01) \\ Anatomie-Chirurgie Orthopédique (42.01) \\ Pharmacologie Clinique (48.03) \\ Néphrologie (52-03) \\ Cardiologie (51.02) \\ Chirurgie Digestive (52.02) \\ Chirurgie Vasculaire (51.04) \\ Anesthésiologie et Réanimation Chirurgicale (48.01) \\ Neurochirurgie (49.02) \\ Pneumologie (51.01) \\ Parasitologie et Mycologie (45.02) \\ Anatomie et Cytologie Pathologiques (42.03) \\ Cancérologie ; Radiothérapie (47.02) \\ Chirurgie Thoracique et Cardio-Vasculaire (51.03) \\ Radiologie et Imagerie Médicale (43.02)
}


M. PAQUIS Philippe

Mme PAQUIS Véronique

M. QUATREHOMME Gérald

M. RAUCOULES-AIME Marc

Mme RAYNAUD Dominique

M. ROBERT Philippe

M. ROSENTHAL Eric

M. SCHNEIDER Stéphane

M. TRAN Albert
Neurochirurgie (49.02)

Génétique (47.04)

Médecine Légale et Droit de la Santé (46.03)

Anesthésie et Réanimation Chirurgicale (48.01)

Hématologie (47.01)

Psychiatrie d'Adultes (49.03)

Médecine Interne (53.01)

Nutrition (44.04)

Hépato-Gastroentérologie (52.01)

\section{PROFESSEURS DEUXIEME CLASSE}

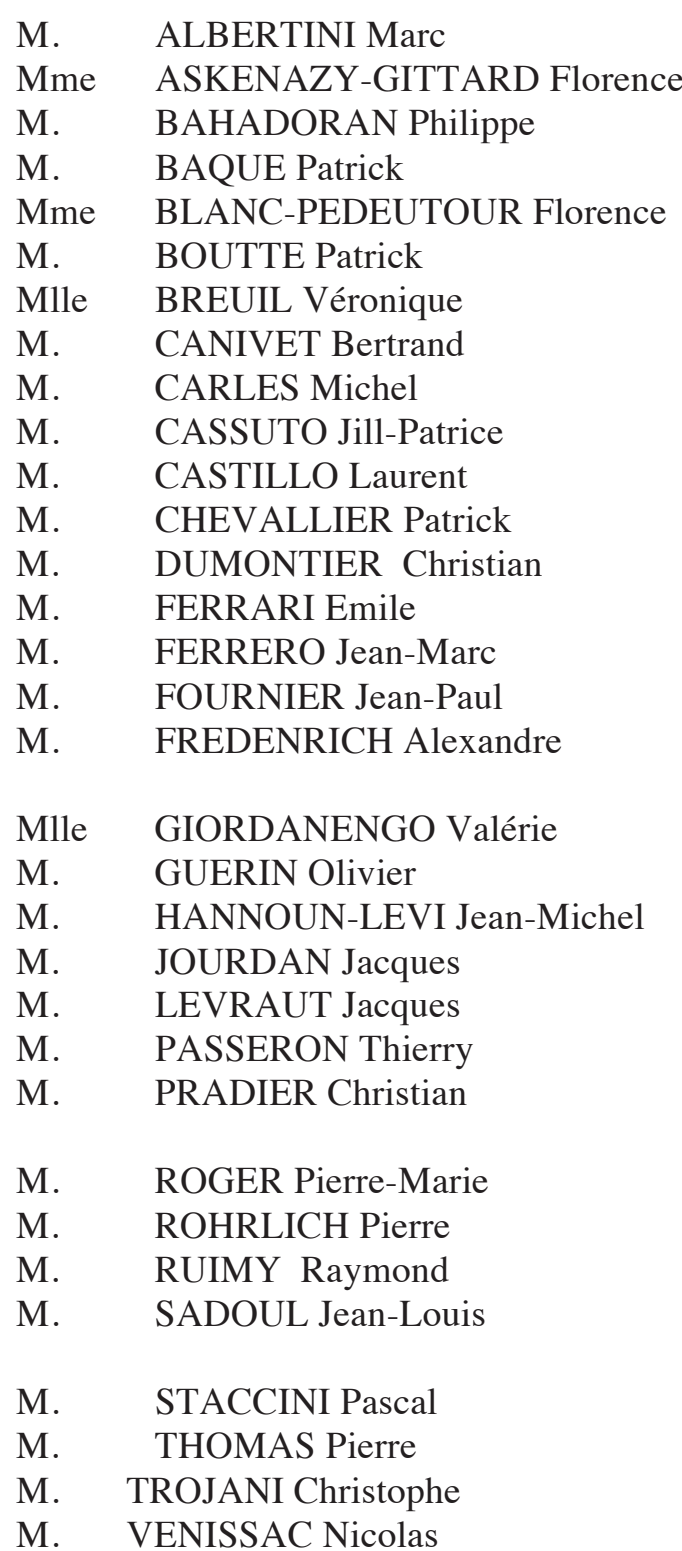

Pédiatrie (54.01)

Pédopsychiatrie (49.04)

Cytologie et Histologie (42.02)

Anatomie - Chirurgie Générale (42.01)

Cancérologie - Génétique (47.02)

Pédiatrie (54.01)

Rhumatologie (50.01)

Médecine Interne (53.01)

Anesthésiologie réanimation (48.01)

Hématologie et Transfusion (47.01)

O.R.L. (55.01)

Radiologie et Imagerie Médicale (43.02)

Chirurgie Plastique (50.04)

Cardiologie (51.02)

Cancérologie ; Radiothérapie (47.02)

Thérapeutique (48-04)

Endocrinologie, Diabète et Maladies métaboliques (54.04)

Bactériologie-Virologie (45.01)

Gériatrie (48.04)

Cancérologie ; Radiothérapie (47.02)

Chirurgie Thoracique et Cardio-Vasculaire (51.03)

Anesthésiologie et Réanimation Chirurgicale (48.01)

Dermato-Vénéréologie (50-03)

Epidémiologie, Economie de la Santé et Prévention (46.01)

Maladies Infectieuses; Maladies Tropicales (45.03)

Pédiatrie (54.01)

Bactériologie - virologie (45.01)

Endocrinologie, Diabète et Maladies Métaboliques (54.04)

Biostatistiques et Informatique Médicale (46.04)

Neurologie (49.01)

Chirurgie Orthopédique et Traumatologique (50.02)

Chirurgie Thoracique et Cardio-Vasculaire (51.03) 


\section{PROFESSEUR DES UNIVERSITES}

M. SAUTRON Jean-Baptiste Médecine Générale

MAITRES DE CONFERENCES DES UNIVERSITES - PRATICIENS HOSPITALIERS

Mme ALUNNI-PERRET Véronique

M. BENIZRI Emmanuel

M. BENOLIEL José

Mme BERNARD-POMIER Ghislaine

M.

Mme BUREL-VANDENBOS Fanny

M. DELOTTE Jérôme

M. DOGLIO Alain

Mme DONZEAU Michèle

M. FOSSE Thierry

M. FRANKEN Philippe

M. GARRAFFO Rodolphe

M. GIUDICELLI Jean

Mme HINAULLT Charlotte

Mlle LANDRAUD Luce

Mme LEGROS Laurence

M. MAGNE Jacques

Mme MAGNIE Marie-Noëlle

Mme MUSSO-LASSALLE Sandra

M. NAÏMI Mourad

M. PHILIP Patrick

Mme POMARES Christelle

Mlle PULCINI Céline

M. ROUX Christian

M. TESTA Jean

M. TOULON Pierre
Médecine Légale et Droit de la Santé (46.03)

Chirurgie Générale (53.02)

Biophysique et Médecine Nucléaire (43.01)

Immunologie (47.03)

Chirurgie Infantile (54-02)

Anatomie et Cytologie pathologiques (42.03)

Gynécologie-Obstétrique (54.03)

Bactériologie-Virologie (45.01)

Biologie du Développement et de la Reproduction (54.05)

Bactériologie-Virologie-Hygiène (45.01)

Biophysique et Médecine Nucléaire (43.01)

Pharmacologie Fondamentale (48.03)

Biochimie et Biologie Moléculaire (44.01)

Biochimie et Biologie Moléculaire ( 44.01)

Bactériologie-Virologie (45.01)

Hématologie et Transfusion (47.01)

Biophysique et Médecine Nucléaire (43.01)

Physiologie (44.02)

Anatomie et Cytologie pathologiques (42.03)

Biochimie et Biologie moléculaire (44.01)

Cytologie et Histologie (42.02)

Parasitologie et Mycologie (45.02)

Maladies Infectieuses ; Maladies Tropicales (45.03)

Rhumatologie (50.01)

Epidémiologie-Economie de la Santé et Prévention (46.01)

Hématologie et Transfusion (47.01)

\section{PROFESSEURS ASSOCIES}

M. DIOMANDE Mohenou Isidore

M. HOFLIGER Philippe

Mme POURRAT Isabelle

Mme. KLEEFIELD Sharon
Anatomie et Cytologie Pathologiques

Médecine Générale

Médecine Générale

Médecine Légale

\section{MAITRES DE CONFERENCES ASSOCIES}

M. GARDON Gilles

M. PAPA Michel
Médecine Générale

Médecine Générale 
PROFESSEURS CONVENTIONNES DE L'UNIVERSITE

M. BERTRAND François

M. BROCKER Patrice

M. CHEVALLIER Daniel

Mme FOURNIER-MEHOUAS Manuella

M. MAGNE Jacques

M. QUARANTA Jean-François
Médecine Interne

Médecine Interne Option Gériatrie

Urologie

Médecine Physique et Réadaptation

Biophysique

Santé Publique 


\section{À notre Maître et Président de Thèse,}

\section{Monsieur le Professeur André BONGAIN,}

Vous me faites le grand honneur de présider ce jury de thèse.

Je vous suis particulièrement reconnaissante de l'intérêt que vous portez à notre formation.

Tout au long de notre internat, nous avons eu la chance de bénéficier de vos grandes connaissances et de votre expérience.

Vous nous avez toujours accueillis avec bienveillance et disponibilité.

Veuillez trouver dans ce travail le témoignage de mon plus profond respect. 
À notre Maître et Juge,

\section{Monsieur le Professeur Patrick BAQUE,}

C'est un grand honneur de vous compter parmi les membres de ce jury.

Nous en sommes d'autant plus honorés que nous savons vos nombreuses obligations hospitalières et universitaires.

Vous me faites l'honneur de juger ce travail, veuillez trouvez ici le témoignage de ma gratitude et de mon profond respect. 
À notre Maître et Juge,

\section{Monsieur le Professeur Jean-Paul Fournier,}

Vous avez accepté spontanément de participer au jury de cette thèse. Je vous en remercie.

$C$ 'est pour moi un honneur que de soumettre mon travail à votre jugement.

Je vous suis reconnaissante de l'attention que vous avez bien voulu y porter.

Recevez l'expression de notre profond respect. 
À notre Maître et Juge,

Monsieur le Docteur Jean BREAUD,

Vous avez d'emblée montré un intérêt certain pour notre travail.

Vous me faites l'honneur de le juger, veuillez trouver ici l'expression de mes sincères remerciements et de mon profond respect. 


\section{À mon Directeur de Thèse,}

\section{Monsieur le Docteur Jérôme DELOTTE,}

Je te remercie d'avoir accepté de diriger ce travail et de m'avoir encouragé et soutenue jusqu'à ce qu'on le mène à bien .

C'est pour moi un honneur particulier que de te compter parmi mon jury de thèse ; quotidiennement, la mesure de tes critiques toujours constructives et argumentées me permet de progresser dans le sens de la compréhension.

Je suis tout particulièrement touchée par la patience et la grande confiance accordées tout au long de l'écriture de ces pages.

Tes compétences et connaissances chirurgicales font de toi un modèle à suivre.

Sois assuré de mon respect le plus sincère. 


\section{A Isabelle BOUCOIRAN,}

Qui m'a fait confiance dés le départ de l'aventure «spatules » et sans qui l'étude n'aurait pu être concrétisée.

Qui a accepté de diriger ce travail à distance depuis le Canada.

Tu m'as accompagnée pendant toute la durée de ce projet avec un professionnalisme irréprochable. Tes compétences statistiques ont sauvé ce projet.

Merci pour tes encouragements perpétuels, ta disponibilité, et ton soutien sans lequel cette thèse n'aurait pas vu le jour! 


\section{A mes parents,}

Supporter l'éloignement géographique qui nous sépare est un combat pour moi de tous les jours.

A mon père,

Ou plutôt au meilleur papa du monde... Tu as toujours cru en moi... et cette phrase que tu ne cesses de répéter " J'AI L'ULTIME CONVICTION... » Je ne l'oublierai jamais. Tu m’as appris à être persévérante. Je t'aime.

\section{A ma maman,}

Ou plutôt à la meilleur maman du monde, à qui je dois mon sourire. La femme qui a fait de moi ce que je suis aujourd'hui. Je t'aime.

\section{A ma sœur Samia,}

Le rôle d'une grande sœur est de prendre soin de sa petite sœur, mais avec Ouidade les rôles sont souvent inversés. Alors merci de prendre soin de moi petite soeur, et surtout de me supporter. Je t'aime.

A ma sœur Safae,

Mon bébé, ma vie, mes yeux. Je t'aime.

A mon frère Nawfal,

J'admire ta zen-attitude quelles que soient les situations. Merci de ton soutien «informatique ». Tu as su être présent dans les moments critiques. Je t'aime.

\section{A Sabah,}

Ma tata, ma deuxième maman, mon amie. J'ai la chance d'avoir la tante la plus cool du monde. A tes enfants Ayman et Anas : mes bébés devenus des hommes aujourd'hui. Je vous aime.

\section{A mes mamies,}

Haja et maman Zineb,

Et leur foi à toutes épreuves. L'amour que vous me portez me comble. 


\section{A la famille Regragui-Benazzi,}

Merci de votre soutien.

Merci Myriam du soutien téléphonique régulier que tu m'apportes. Et j'adore quand tu m'appelles Maryline, je me sens toujours un peu star à ce moment là.

Tata Khadija, merci pour ta bonté, tes pensées.

Merci Ismahane pour ta gentillesse, ta bonne humeur et ton humour

\section{A la famille du Maroc,}

Les Sbiti : Hanane, Amina, Tonton Zouhir, Tonton Saïd et leurs enfants

Merci de prendre soin de moi comme vous le faites lorsque je suis à vos côtés au Maroc. Vos petites attentions me touchent beaucoup. C'est toujours une joie de vous retrouver, et le déchirement quand je vous quitte.

La famille de Tizimi : merci pour votre gentillesse soutien et légendaire accueil que vous me faites chaque année.

La famille Aidouni/Salahdine : merci pour l'ambiance festive qui règne quand je suis à vos côtés. C'est toujours une joie de vous retrouver.

Aux voisins et voisines du quartier de Béni M’Ahmed Derb Larbi Boulissi qui sont pour moi plus que des amis.

\section{A mes amis de toujours,}

Merci de votre gentillesse et simplicité. Votre soutien m'est très cher.

La famille Agbal,

La famille Dahbi

La famille Fougani

\section{A ma meilleure amie Soraya,}

Parce qu'aucune autre amie me connaît aussi bien que toi, tu lis en moi comme dans un livre.

Ta présence constante dans les moments difficiles m'apporte beaucoup de réconfort et d'assurance. Et vive la Kabylie! (La seule fois où tu l'entendras de ma bouche...)

\section{A Gaël mon ami,}

Tu es unique dans ton genre, ne change jamais. Parce qu'avec toi on ne s'ennuie jamais. Nos petites séances « ragots » me manquent et ton humour toujours très recherché aussi! Ta

Dadouze.

\section{A mon amie Amatola,}

Absente ce jour mais présente dans mon cœur. 
A Sandra,

Nos fous rires me manquent. Et mon amie aussi.

A Hafida,

Merci pour tes pieux conseils.

A Myriam Aazzabi,

Et nos petites descentes à Marrakech...

A Cédric et Camille,

Mes amis de fac, que je n'oublierai jamais.

A Alexandra Bonjour,

Merci pour ces moments passés avec toi.

A Elodie Vuvu,

Et nos soirées inoubliables.

A Adrien et Gwennolee,

A nos aventures fréjussiennes passées.

\section{A mes amies Gynéco-pouff,}

Constance, qui restera pour toujours MA CO-INTERNE. Nos virées chez Fit-Fit n'ont pas de prix et il en vaut de même pour notre amitié. J'apprécie être ta grande sœur lorsque tu me le demandes. Je serai toujours là pour toi.

Tatiana, ton humour et ton style vestimentaire m'inspirent. Ton amitié m'est chère.

Marine, ma quinqounette. Ton esprit carré est un modèle à suivre pour moi et ce vers quoi je souhaite tendre.

Charline, alias Chachou, tes petites charlinites me manquent, mais pas que ça! toi aussi...

Mélanie, Caroline, Julie. Votre bonne humeur est « salement « caractéristique. Je vous valide.

Aux cointernes, Bérengère, Alexis, Clélia, Jo, Delphine, Faustine,...

Mes co-internes actuelles, Fanny, Julia, Justine. Merci de votre soutien quotidien. Et merci d'avoir ménagé mon temps afin de finir la thèse.

\section{A mes chefs,}

Le Dr Samir, alias «khoya Samir», Merci de ta disponibilité, tes conseils qui m'aident à avancer. Tu es comme un grand frère.

Le Dr Lyna, ta douceur, ta gentillesse, ton écoute m'apaisent quand je suis avec toi. 
Le Dr Sarah Nataf, ma révélation 2013. A nos petites soirées entre filles et nos pauses SDN Archet -2.

Le Dr Magali Hilmi, tu es pour moi l'obstétricienne par excellence. Merci de m'avoir laissé réaliser ma première césarienne.

Le Dr Maxence, merci pour ton sens de la pédagogie.

Le Dr Olivier, merci pour ta perpétuelle bonne humeur, et disponibilité.

Le Dr Galeazzi, ces séances maquillage pendant les gardes en CHU niveau 3 resteront gravées dans ma mémoire à jamais.

Le Dr Muzelle, ta disponibilité à toute épreuve est rare. Merci.

Le Dr Cynthia Trastour, tu es unique et on a beaucoup à apprendre de toi!

Le Dr Clerté, merci de nous faire partager votre expérience d'obstétricien chevronné.

Le Dr Duforestel, merci de m'avoir appris les bases de chirurgie.

\section{A l'équipe cannoise,}

Le Dr Toulalan, merci pour ton enseignement et ton humour $10^{\text {ème }}$ degré.

Le Dr D’Angelo, le Dr Sans-Michel, le Dr De Jésus et le Dr Vignal, travailler avec vous est un réel plaisir.

\section{A l’équipe sages-femmes du CHU,}

Sophie, Marianne, Maria, Clémence, Manue, Florence, Florianne, Micka, Laura, Magalie... Je n'oublierai jamais ces gardes au CHU, entre détentes et coups de stress.

\section{A l'équipe AS du CHU,}

Ma Samia d'amour, la jolie Naïma, Nathalie,Leila (et nos petites discussions de nanas) Capucine, Pamela et Mag et toutes nos pauses en salle 7...

\section{A l'équipe Anesthésistes du CHU,}

Le Dr Lallia, le Dr Carlon, le Dr Maslowski, Flo, merci pour les pauses Nespresso...

\section{Aux secrétaires de l’Archet,}

Steph, Christelle, Sandrine, Yvette, Julie et les autres ! 


\section{A l'équipe Bliss Master Coulisses,}

Alix, Mylène, Cécile, Marie, Popo, Djegueur,Yo...

\section{Aux Steppeuses,}

Merci à Laeti de m'avoir fait progresser dans le step, qui représente plus qu'un sport à mes yeux, une échappatoire. LE STEP C'EST MA VIE !!!!

Et à mes copines du step, devenues amies au fil de nos séances plus qu'euphorisantes : ma Isabelle, ma Amanda, ma Laeti Pepsi, ma Michèle, ma Julie, etc. A nos prochaines conventions! Merci à Jérôme pour ses mix hors du commun.

\section{Et aux nouvelles rencontres,}

A Réda, merci d'être tout simplement là, pour aujourd'hui et pour demain.

\section{Enfin à toutes ces femmes, ces mères, ces patientes,}

Grâce auxquelles ce métier est un bonheur chaque jour! Merci! 


\section{ABREVIATIONS}

ST : Spatules de Thierry

EI : Extractions instrumentales

CEGO : Collège Européen de Gynécologie Obstétrique

CNGOF : Collège National des Gynécologues et Obstétriciens français

CHU : Centre hospitalier universitaire

DES : Diplôme d'études spécialisées

GO : Gynécologie obstétrique

AUDIPOG : Association des Utilisateurs de Dossiers Informatisés en Pédiatrie, Obstétrique et Gynécologie

NP : Niveau de preuve

SOGC : Society of Obstetricians and Gynaecologists of Canada

RANZCOG : The Royal Australian and New Zealand College of Obstetricians and Gynaecologists

RCOG : Royal College of Obstetricians and Gynaecologists

RPC : Recommandations pour la pratique clinique

HAS : Haute Autorité de Santé

OR : Odd ratio

IC : Intervalle de confiance

OMS : Organisation mondiale de la santé

OIGA : Occipito iliaque gauche antérieure

OIDP : Occipito iliaque droite postérieure

OIDA : occipito iliaque droite antérieure

OS : Occipito-sacré

OP : Occipito-pubien

OIGT : Occipito iliaque gauche transverse

OIDT : Occipito iliaque droite transverse

ODG : Obstétricien de garde

MLM : Modèle linéaire mixte 
EBCOG : European Board and College of Obstetricians and Gynaecologists AGOF : Association des gynécologues obstétriciens en formation 


\section{INDEX DES FIGURES}

Figure 1 : Données statistiques du mode d'accouchement des mères entre 1994 et 2008.

Figure 2 : Représentation schématique du diaphragme pelvien (vue inférieure).

Figure 3 : Vue d'ensemble des muscles du périnée.

Figure 4 : Ampliation du périnée au cours de l'accouchement.

Figure 5 : Les spatules de Thierry.

Figure 6 : Principes d'action des spatules.

Figure 7 : Spatules en place selon l'axe sous-occipito-mentonnier.

Figure 8 : L'effet levier des spatules.

Figure 9 : Modification de l'axe des spatules lors de la progression de la présentation.

Figure 10 : La ventouse Kiwi ${ }^{\circledR}$.

Figure 11 : Zone d'action de la ventouse obstétricale sur le cuir chevelu.

Figure 12 : Traction dans l'axe ombilicococcygien.

Figure 13 : Forceps de Tarnier.

Figure 14 : Un nouvel instrument d'extraction : l'Odon Device. 
Figure 15 : Répartition géographique régionale des taux d'extractions instrumentales en CHU de niveau III en 2007.

Figure 16 : Distribution géographique régionale de la part des spatules dans l'extraction instrumentale en CHU de niveau III en 2007.

Figure 17 : Distribution géographique régionale de la part des forceps dans l'extraction instrumentale en CHU de niveau III en 2007.

Figure 18 : Distribution géographique régionale de la part des ventouses dans l'extraction instrumentale en CHU de niveau III en 2007.

Figure 19 : Diagramme de sélection des extractions par spatules évaluées (Résultats de la $1^{\text {ère }}$ phase de notre étude).

Figure 20 : Nombre d'extractions évaluées en fonction de l'ancienneté des internes (Résultats de la $1^{\text {ère }}$ phase de notre étude).

Figure 21 : Évaluation par l'obstétricien de garde du niveau de compétence globale de l'interne pour la réalisation des accouchements vaginaux assistés par spatules de Thierry (Résultats de la $1^{\text {ère }}$ phase de notre étude).

Figure 22 : Taux de lésion périnéale sévère en fonction du nombre de semestres validés en gynécologie obstétrique (Résultats de la $1^{\text {ère }}$ phase notre étude).

Figure 23 : Evolution des scores totaux des internes évalués par les obstétriciens de garde en fonction de l'ancienneté des internes (Résultats de la $2^{\text {ème }}$ phase notre étude).

Figure 24 : Niveau de compétence globale de l'interne selon l'obstétricien de garde (Résultats de la $2^{\text {ème }}$ phase notre étude).

Figure 25 : Répartition du niveau de confiance de l'interne en fonction de son ancienneté (Résultats de la $2^{\text {ème }}$ phase notre étude). 
Figure 26 : Répartition des scores de l'évaluation des internes par le senior en fonction du seuil de réalisation des 20 extractions par spatules (Résultats de la $2^{\text {ème }}$ phase notre étude).

Figure 27 : Schéma théorique des courbes d'apprentissage.

Figure 28 : Courbes d'apprentissage théorique de l'anesthésie locorégionale en fonction du degré de formation préalable et des moyens utiles à ce stade.

Figure 29 : Variation de la vitesse d'apprentissage après mise en situation sur simulateurs.

Figure 30 : The BirthSIM Simulator.

Figure 31 : Description du modèle d'apprentissage : les étapes du modèle de Butler.

Figure 32 : Laerdal : console de paramétrage SimMom ${ }^{\circledR}$. 


\section{INDEX DES TABLEAUX}

Tableau 1 : Indications des extractions instrumentales.

Tableau 2 : Prérequis avant la réalisation d'une extraction instrumentale.

Tableau 3 : Niveaux de preuve scientifique et grades des recommandations.

Tableau 4 : Classifications française et OMS des déchirures périnéales obstétricales et schémas correspondants.

Tableau 5 : Synthèse des avantages et limites des spatules.

Tableau 6 : Protocole d'étude du nouvel instrument d'extraction : l'Odon Device.

Tableau 7 : Caractéristiques, maternelles, obstétricales et néonatales de la première phase de l'étude (mai à novembre 2010).

Tableau 8 : Résultats à chaque item de la grille d'évaluation des internes par l'obstétricien de garde (1ere phase d'étude).

Tableau 9 : Comparaison des taux de complications maternelles et néonatales des accouchements assistés par spatules de Thierry en fonction de l'ancienneté de l'interne, de son niveau de compétence globale évalué par l'obstétricien de garde, de son niveau de confiance au moment de l'extraction et la difficulté de la procédure.

Tableau 10 : Caractéristiques, maternelles, obstétricales et néonatales de la seconde phase de l'étude (novembre 2010 à mai 2012).

Tableau 11: Résultats à chaque item de la grille d'évaluation des internes par l'obstétricien de garde (2ème phase d'étude). 
Tableau 12 : Nature et nombre de gestes à réaliser pendant l'internat de gynécologie obstétrique . 


\section{TABLE DES MATIERES}

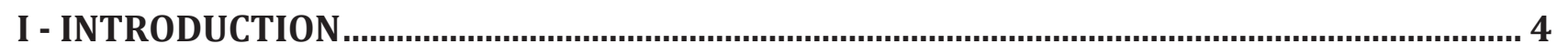

II - LES EXTRACTIONS INSTRUMENTALES..................................................................... 6

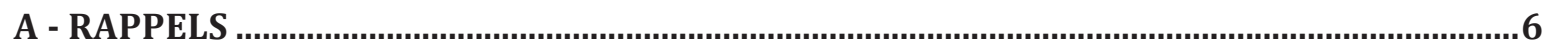

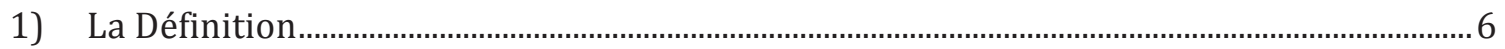

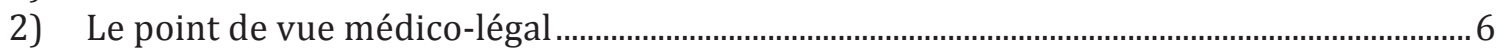

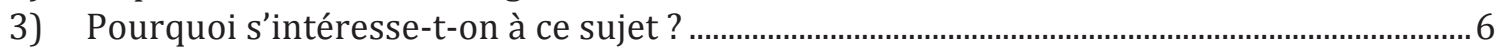

4) Quelles sont les indications des extractions instrumentales ?................................................

5) Quelles sont les contre-indications des extractions instrumentales ? ................................ 10

6) Quel est le pré requis avant la réalisation d'une extraction instrumentale ? .................... 10

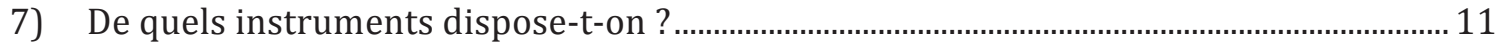

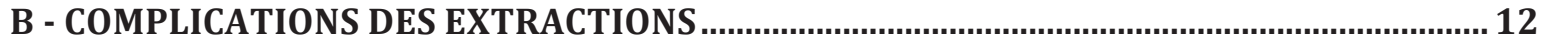

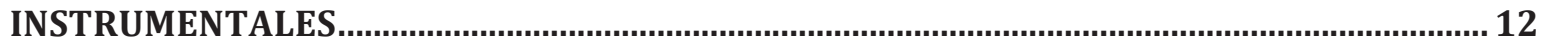

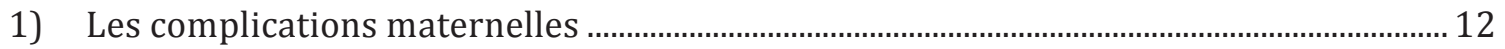

2) Les complications néonatales des extractions instrumentales ..........................................23

C - LES SPATULES DE THIERRY ........................................................................................................ 25

1) Description de l'instrument ...................................................................................................... 25

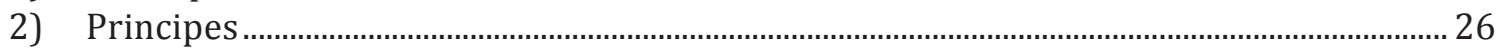

3) Description du geste d'extraction............................................................................................ 27

4) Avantages et limites des spatules de Thierry ......................................................................... 31

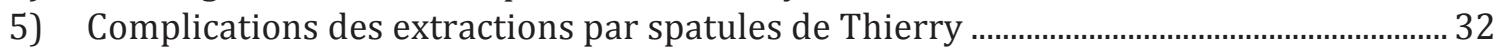

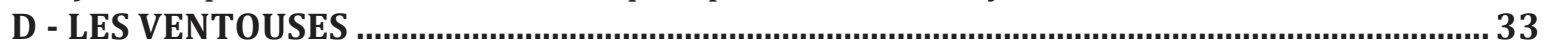

1) Description de l'instrument ..................................................................................................... 33

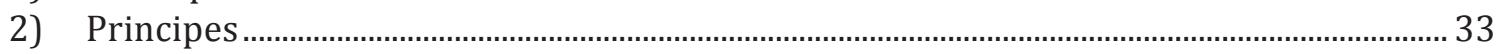

3) Description du geste d'extraction................................................................................................. 34

4) Avantages et limites des ventouses obstétricales ................................................................ 36

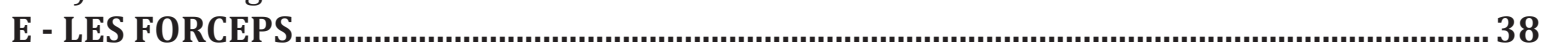

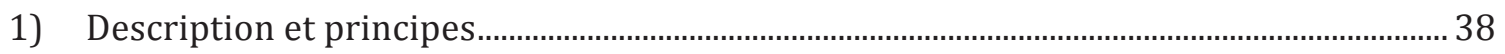

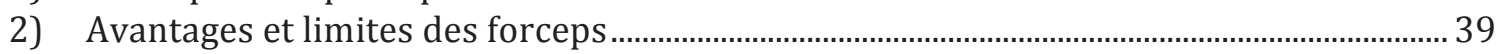

F - L'ODON DEVICE : UN NOUVEL INSTRUMENT D'EXTRACTION FOETALE ......................40

G - EPIDEMIOLOGIE : QUELS INTRUMENT UTILISE-T-ON EN FRANCE ?........................... 43

1) Distribution géographique du taux d'extraction instrumentale en CHU de niveau III en

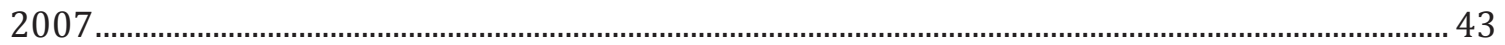

2) Distribution géographique régionale de la part des spatules dans l'extraction instrumentale en CHU de niveau III en 2007 ................................................................................... 44

3) Distribution géographique régionale de la part des forceps dans l'extraction instrumentale en CHU de niveau III en 2007. .................................................................................... 45

4) Distribution géographique régionale de la part des ventouses dans l'extraction instrumentale en CHU de niveau III en 2007 ................................................................................... 46

H - QUEL INSTRUMENT FAUT-IL APPRENDRE? ............................................................. 47

1) Que recommande le Collège National des Gynécologues et Obstétriciens français ?.... 47

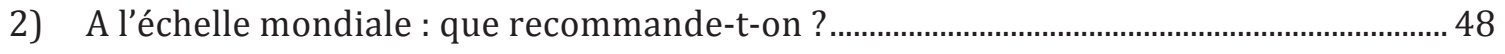

III - MATERIELS ET METHODES . .

B - PREMIERE PHASE DE L'ETUDE............................................................................................ 50

C - DEUXIEME PHASE DE L'ETUDE........................................................................................ 50

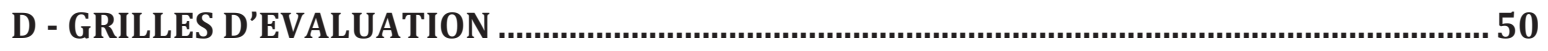


C - RECUEIL DES DONNEES

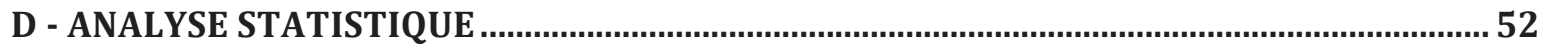

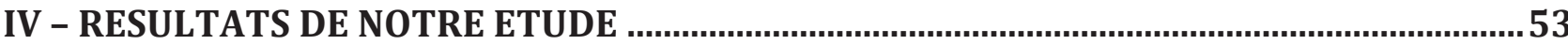

A - RESULTATS DE LA 1ERE PHASE DE L'ETUDE ….......................................................... 53

1) Description : caractéristiques maternelles, obstétricales et néonatales ............................. 54

2) Profil des internes évalués............................................................................................................. 56

3) Auto-questionnaire des internes ............................................................................................ 57

4) Évaluations par les obstétriciens de garde .............................................................................. 57

5) Lien entre ancienneté de l'interne, évaluations et complications maternelles et néonatales

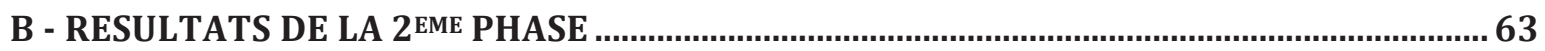

1) Description : caractéristiques maternelles, obstétricales et néonatales ............................ 63

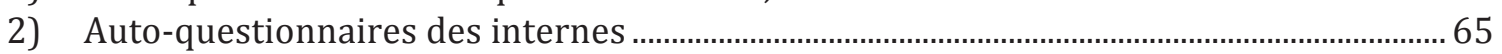

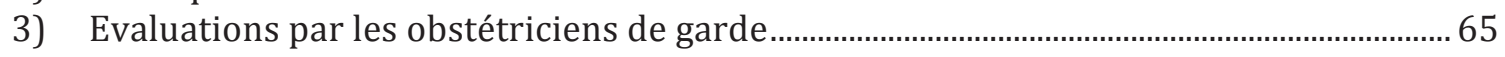

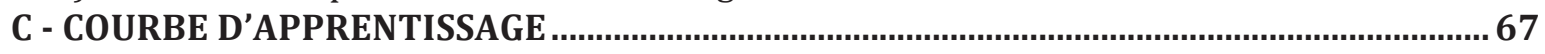

1) Impact de l'ancienneté de l'interne sur leurs résultats aux évaluations ...............................67

2) Impact du niveau d'expérience des internes sur leurs résultats aux évaluations............69

3) Niveau de confiance de l'interne en fonction de l'ancienneté et du seuil des 20

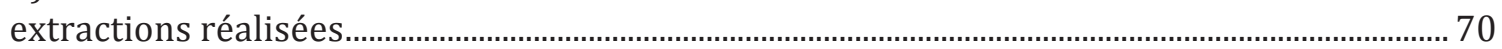

$\mathrm{V}$ - DISCUSSION

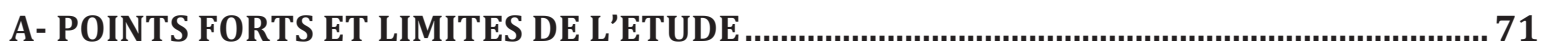

B - INTERPRETATION DES RESULTATS DE LA PREMIERE PHASE DE NOTRE ETUDE ...... 72

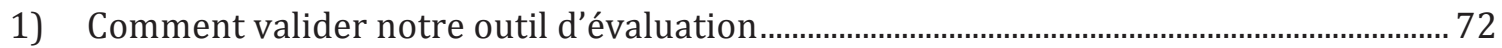

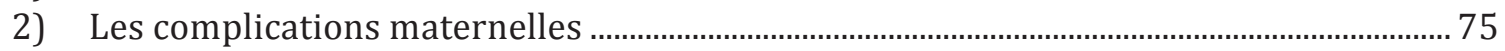

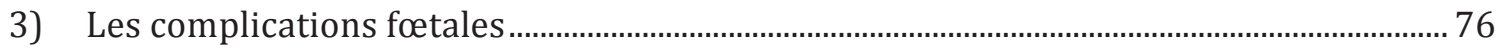

C - INTERPRETATION DES RESULTATS DE LA 2EME PHASE DE NOTRE ETUDE ................77

1) L'intérêt des courbes d'apprentissage en obstétrique ......................................................... 77

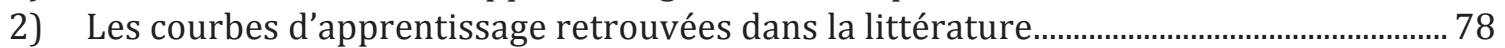

3) La courbe d'apprentissage des extractions par forceps ......................................................... 80

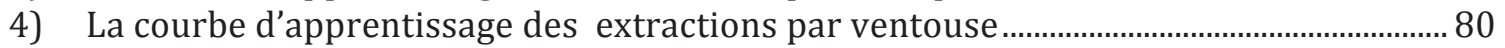

5) Combien d'extractions faut-il réaliser pendant l'internat de gynécologie obstétrique ? 80

6) La courbe d'apprentissage des extractions par spatules de Thierry ....

7) L'intérêt des simulateurs obstétricaux pour améliorer la courbes d'apprentissage des extractions instrumentales

D - EVALUATION DE LA FORMATION DES INTERNES EN GYNECOLOGIE OBSTETRIQUE 84

1) Evaluation des compétences de l'interne de gynécologie obstétrique.................................84

2) Les objectifs de la formation aux extractions instrumentales ............................................. 85

3) De quels moyens dispose-t-on pour former l'interne en gynécologie obstétrique aux

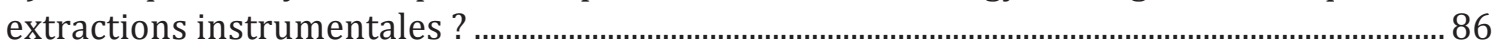

4) Comment évaluer cette formation ? ...................................................................................... 91

5) Améliorer la formation en gynécologie obstétrique grâce à la simulation......................... 94

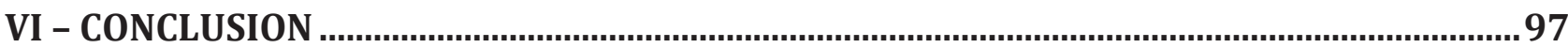

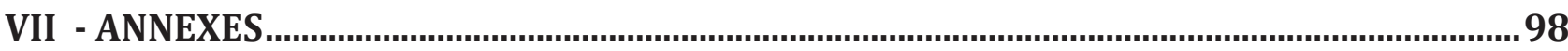

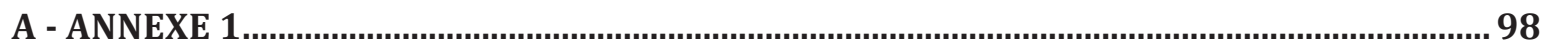

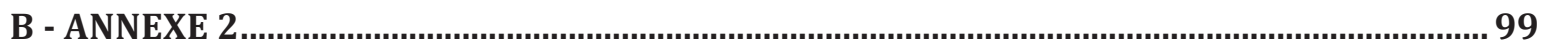

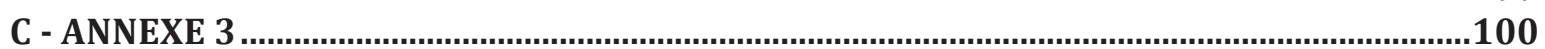

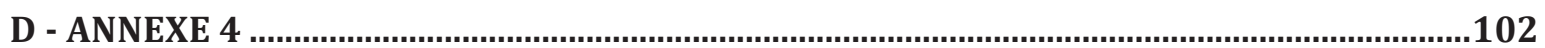

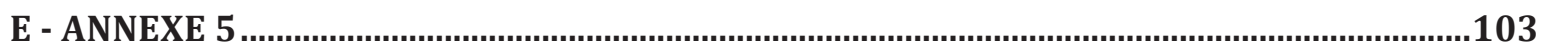

F - PUBLICATION SOUMISE ………………...................................................................104

VIII - REFERENCES .............................................................................................................. 120 
A - FIGURES

B - BIBLIOGRAPHIE 


\section{I - INTRODUCTION}

Les spatules de Thierry (ST) sont un instrument d'extraction fœtale mis au point dans les années 1950 par le Docteur E. Thierry.

Cet instrument est basé sur un principe mécanique d'orientation et de propulsion du mobile fœtal, chaque cuillère agissant comme un levier qui prend appui sur les parties molles maternelles et sur l'os malaire fœtal.

Certains de ses avantages sont bien connus des utilisateurs : rapidité de mise en place et protection du pôle fœtal. Les taux de lésions néonatales reportées sont extrêmement faibles, voire exceptionnels pour les ST de l'ordre de $0,3 \%$ pour les complications les plus graves [1, 2,3 ] contre $1,1 \%$ pour les forceps et $0,76 \%$ pour les ventouses [4].

Cependant, le mécanisme d'action de cet instrument est basé sur la propulsion, ce qui peut se traduire par une pression sur les tissus périnéaux féminins et pourrait augmenter le risque de lésions périnéales sévères par rapport aux autres instruments d'extractions [5,6].

L'absence d'essai randomisé contrôlé comparant l'usage des ST aux autres instruments d'extraction ne permet pas de chiffrer ce sur risque, cependant les données des études portant sur les extractions par spatules sont rassurantes, avec des taux de lésions périnéales sévères de variant de 2,1 à $17,4 \%$.[1,2,7-14].

En raison d'une faible diffusion de l'apprentissage des extractions par ST en dehors de sa zone de création, elles restent essentiellement utilisées dans le sud de la France et peu connues par ailleurs $[5,6]$.

La validation de l'apprentissage des étudiants en médecine semble une donnée essentielle pour éviter des complications liées aux extractions instrumentales (EI).

Selon le Collège Européen de Gynécologie Obstétrique (CEGO), le cursus d'apprentissage des extractions instrumentales par les étudiants en Médecine doit comporter au moins 40 extractions instrumentales, toutes modalités incluses.

Cependant la valeur seuil du nombre de procédures à réaliser en fonction de l'instrumentation choisie n'est définie ni par le CEGO [15] ni parle Collège National des Gynécologues 
Obstétriciens Français (CNGOF) [16]. Si, en 1964, LLyod avait proposé un seuil de 20 procédures d'extractions par spatules pour être jugé compétent, à l'heure actuelle, il n'existe pas de données sur la courbe d'apprentissage des extractions par ST [17].

Pourtant, cette donnée est essentielle à connaître afin de déterminer les besoins en terme d'apprentissage des étudiants dans nos maternités et d'adapter en conséquence les méthodes d'apprentissage des extractions par spatules. Une étude rétrospective réalisée dans notre établissement, le centre hospitalier universitaire (CHU) de Nice avait mis en évidence un risque augmenté de lésions périnéales sévères lorsque les extractions étaient réalisées par des jeunes internes par rapport à celles réalisées par des internes en cinquième semestre et plus [12].

L'objectif de ce travail est double : établir un outil d'évaluation des extractions par ST et définir la courbe d'apprentissage des extractions par cet instrument pour en déduire si le seuil de 20 procédures est suffisant pour permettre la réalisation autonome de ces extractions. 


\section{II - LES EXTRACTIONS \\ INSTRUMENTALES}

\section{A - RAPPELS}

\section{1) La Définition}

L' EI du foetus est l'art de donner naissance par les voies naturelles à un enfant vivant grâce à l'utilisation d'un moyen mécanique.

Cette définition exclut donc les manœuvres manuelles, les embryotomies et les EI à travers une hystérectomie abdominale [18].

Le principe est de raccourcir la période d'expulsion, d'aider à la flexion et/ou compléter la rotation de la tête fœtale [16].

\section{2) Le point de vue médico-légal}

L'utilisation des instruments obstétricaux d'extraction, quels qu'ils soient, est réservée aux docteurs en médecine, à l'exclusion donc des sages-femmes aussi compétentes puissent-elles être [18]. L'usage veut que cette utilisation soit le fait exclusif des docteurs en médecine titulaires d'une qualification suffisante, c'est-à-dire, au minimum du certificat d'études spéciales d'obstétrique dans l'ancien régime des études médicales, d'un internat qualifiant en gynécologie obstétrique (GO) ou aujourd'hui de l'obtention du diplôme d'études spécialisées ( DES) de GO.

\section{3) Pourquoi s'intéresse-t-on à ce sujet ?}

Il existe de nombreuses publications concernant les EI, et l'intérêt porté à ce sujet se justifiait par les arguments suivants :

- une jurisprudence de moins en moins tendre pour les responsables d'incidents de la voie basse, pouvant être dramatiques [19]. 
- l'enseignement des techniques de la voie basse considéré très long et douloureux par compagnonnage, nécessitant une disponibilité de tous les instants, à tous les niveaux, économiquement non rentable à court terme.

- l'image catastrophique des instruments obstétricaux auprès d'un public désinformé, rêvant d'accouchements exotiques et encore marqué par les drames de l'obstétrique d'avant l'ère des antibiotiques présents dans les mémoires des anciennes générations de parents [20].

- l'intérêt porté pour le bien-être fœtal appréhendé de manière scientifique par des études de cohortes sur le devenir des nouveau-nés et que ces études sont toutes en faveur du raccourcissement raisonnable de la phase d'expulsion [21,22].

- la volonté de réduire le taux de césarienne.

- si la santé n'a pas de prix, la naissance en a elle de multiples, de la durée d'hospitalisation aux vécus d'échecs de mères dont le devenir des enfants dira ce qu'ils auront coûté.

L'EI [16] :

- reste un mode d'assistance à l'accouchement fréquent quoi que en légère diminution en France : 11\% des naissances en 2008 [23] [Figure 1].

- doit minimiser le risque de morbidité pour la mère et le foetus ;

- suppose le choix de l'instrument pour lequel on a reçu une formation et dont on a l'expérience;

- nécessite le respect des indications et des règles d'application.

- doit en principe être réservée aux situations où l'EI facile est anticipée. 


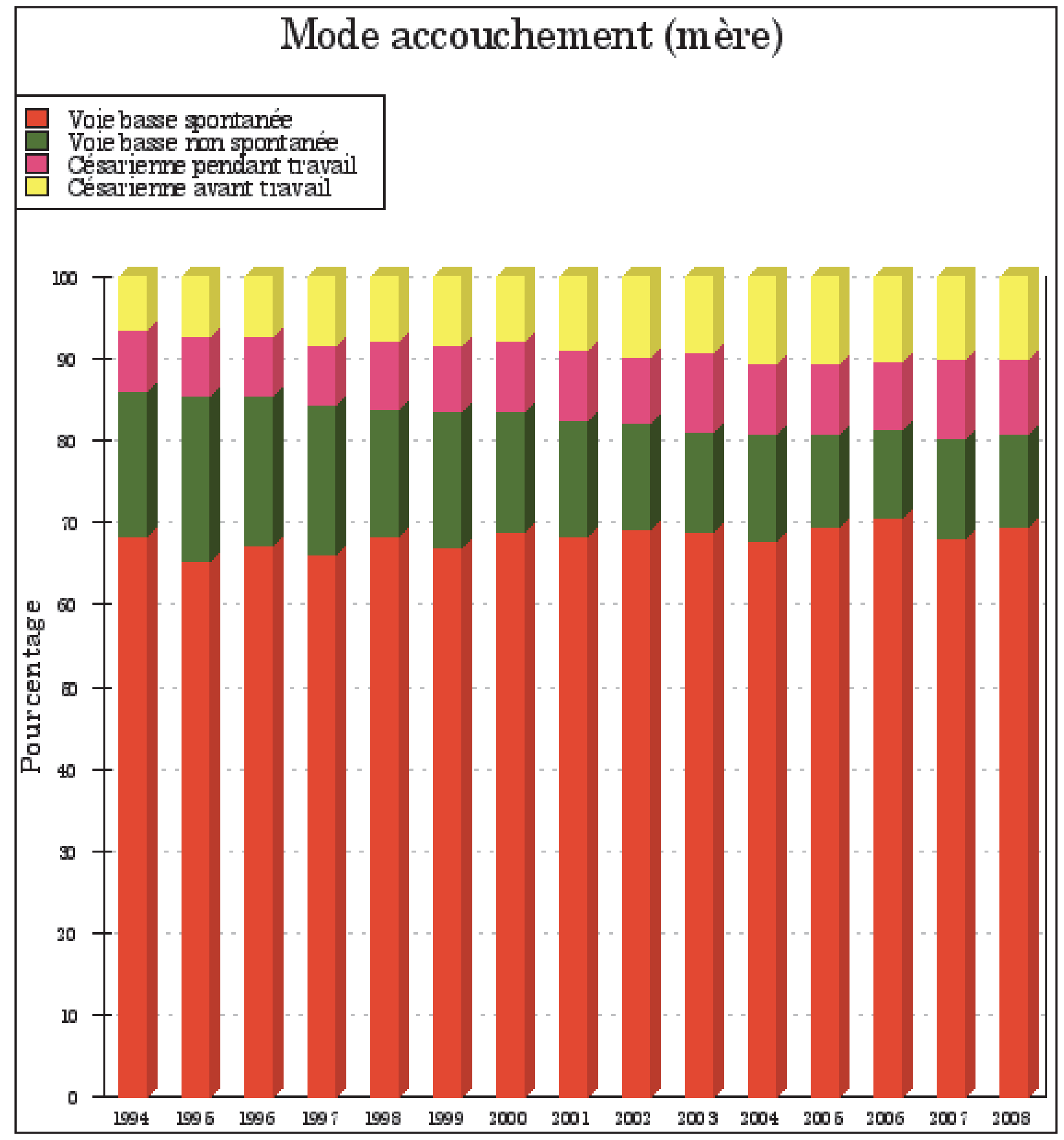

Figure 1 : Données statistiques du mode d'accouchement des mères entre 1994 et 2008. 


\section{4) Quelles sont les indications des extractions instrumentales?}

Elles sont d'ordre fœtales, maternelles, ou en cas d'anomalie de la seconde phase du travail [Tableau 1] [24]. Nulle indication n'est absolue et chaque situation doit être évaluée au cas par cas.

Tableau 1 : Indications des extractions instrumentales.

\begin{tabular}{|c|l|}
\hline Type d'indication & Indications \\
\hline Foetale & Anomalie du rythme cardiaque foetal \\
\hline Maternelles & Fatigue maternelle \\
& $\begin{array}{l}\text { Pathologie contre-indiquant les manœuvres de } \\
\text { vasalva : insuffisances cardiaques sévères, } \\
\text { malformations vasculaires cérébrales non } \\
\text { traitées, myopathies invalidantes, antécédents } \\
\text { de décollement de rétine et de pneumothorax }\end{array}$ \\
\hline Anomalie de la seconde phase du travail & $\begin{array}{l}\text { l'intensité des efforts expulsifs a été jugée } \\
\text { suffisante } \\
\text { d'efforts expulsifs sans progression du mobile }\end{array}$ \\
\hline & $\begin{array}{l}\text { Absence d'accouchement après 30 minutes } \\
\text { foul avec un RCF normal dans la mesure où }\end{array}$ \\
\hline
\end{tabular}

Il est impératif qu'avant toute EI, l'obstétricien, quelle que soit l'urgence de la situation, prenne le temps de se poser deux questions fondamentales, étroitement imbriquées entre elles : 
- L'EI est-elle la réponse adaptée à la situation, en évaluant la balance bénéfice/risque de cette action versus une autre (réalisation d'une césarienne, mise en œuvre ou poursuite des efforts expulsifs, mais aussi attitude expectative) ?

- Les conditions médicales et environnementales réunies permettent-elles la réalisation d'une EI dans les conditions optimales de sécurité pour l'enfant comme pour la mère ?

\section{5) Quelles sont les contre-indications des extractions instrumentales ?}

\section{En cas de suspicion de disproportion foeto-pelvienne :}

Il n'existe aucune étude concernant le pronostic néonatal et/ou maternel après réalisation d'EI alors qu'était suspectée une disproportion foeto-pelvienne. Les sociétés savantes ne retiennent pas cette situation comme une contre-indication absolue, du fait de sa part de subjectivité [24].

En cas présentation engagée partie haute : il n'existe pas actuellement d'arguments de haut niveau de preuve dans la littérature pour contre-indiquer formellement la réalisation d'une EI à la partie haute, mais elle n'est pas recommandée (accord professionnel) (Niveau de Preuve (NP) 4) $[16,25,26]$.La situation sera analysée au cas par cas et en fonction de l'expérience du praticien (avis d'expert) [25,26] .Les obstétriciens doivent avoir conscience que dans 6\% des cas , ils sont susceptibles de diagnostiquer à tort une présentation engagée à la partie haute en particulier en présence d'une bosse séro sanguine (NP2)[27] .

\section{6) Quel est le pré requis avant la réalisation d'une extraction instrumentale ?}

Il n'existe que très peu de données scientifiques de niveau de preuve élevé dans la littérature concernant les prérequis des EI. Les conditions suivantes ont été adaptées des recommandations de la SOGC (Society of Obstetricians and Gynaecologists of Canada) [28], du RANZCOG (The Royal Australian and New Zealand College of Obstetricians and Gynaecologists) [29] et du RCOG (Royal College of Obstetricians and Gynaecologists) [26] [Tableau 2]. 


\section{7) De quels instruments dispose-t-on ?}

Les spatules : les spatules de Thierry et de Tessier

Les ventouses

Les forceps

Un nouvel instrument : l'Odon Device

Tableau 2 : Prérequis avant la réalisation d'une extraction instrumentale.

\begin{tabular}{|ll|}
\hline \multicolumn{1}{|c|}{ Prérequis avant la réalisation d'une extraction instrumentale } \\
$\checkmark \checkmark$ & Connaissance du dossier obstétrical \\
$\checkmark$ & Dilatation complète \\
$\checkmark$ & Présentation engagée \\
$\checkmark$ & Bassin cliniquement examiné \\
$\checkmark$ & Positionnement exact de la tête fotale connu \\
$\checkmark$ & Analgésie maternelle adéquate \\
$\checkmark$ & Membranes rompues \\
$\checkmark$ & Vessie maternelle vide \\
$\checkmark$ & Intervention médicale expliquée à la patiente et au personnel soignant \\
$\checkmark$ & Choix de l'instrument en fonction de la situation obstétricale et des préférences de \\
$\checkmark$ & Réalisation de l'EI en présence de la sage-femme et si possible d'une autre personne \\
$\checkmark$ & Asepsie rigoureuse \\
& l'obstétricien
\end{tabular}




\section{B - COMPLICATIONS DES EXTRACTIONS INSTRUMENTALES}

\section{1) Les complications maternelles}

\section{a) Généralités}

Il existe de nombreuses publications concernant les complications maternelles des EI.

Les recommandations pour la pratique clinique (RPC) du CNGOF en 2008 les rappellent et en font une synthèse [16].

Selon le niveau de preuve des études sur lesquelles elles sont fondées, les recommandations ont un grade variable, côté de $\mathrm{A}$ à $\mathrm{C}$ selon l'échelle proposée par la Haute Autorité de Santé (HAS) [30,31] [Tableau 3].

Tableau 3 : Niveaux de preuve scientifique et grades des recommandations.

\begin{tabular}{|c|c|}
\hline $\begin{array}{l}\text { Niveau de preuve scientifique fourni par la littérature } \\
\text { (études thérapeutiques) }\end{array}$ & Grade des recommandations \\
\hline $\begin{array}{l}\text { Niveau } 1 \text { (NP1) } \\
\text { - Essais comparatifs randomisés de forte puissance. } \\
\text { - Méta-analyse d'essais comparatifs randomisés } \\
\text { - Analyse de décision basée sur des études bien menées }\end{array}$ & $\begin{array}{l}\text { Preuve scientifique établie } \\
\text { A }\end{array}$ \\
\hline $\begin{array}{l}\text { Niveau } 2 \text { (NP2) } \\
\text { - Essais comparatifs randomisés de faible puissance. } \\
\text { - Études comparatives non randomisées bien menées } \\
\text { - Études de cohorte }\end{array}$ & $\begin{array}{l}\text { Présomption scientifique } \\
\text { B }\end{array}$ \\
\hline $\begin{array}{l}\text { Niveau } 3(\mathbf{N P 3}) \\
\text { Études cas-témoins }\end{array}$ & \\
\hline $\begin{array}{l}\text { Niveau } 4 \text { (NP4) } \\
\text { - Études comparatives comportant des biais importants } \\
\text { - Études rétrospectives } \\
\text { - Séries de cas }\end{array}$ & $\begin{array}{l}\text { Faible niveau de preuve } \\
\text { C }\end{array}$ \\
\hline
\end{tabular}




\section{$>$ Complications immédiates et à court terme}

L'extraction instrumentale augmente significativement le risque de déchirures périnéales sévères ( $3 \mathrm{e}$ et $4 \mathrm{e}$ degré) par rapport à la voie basse spontanée (grade $\mathrm{B}$ ). Toutes les études de cohortes concluent que la réalisation d'une EI majore les risques de déchirures périnéales sévères par rapport à la voie basse spontanée.

Leurs résultats sont souvent difficilement comparables en raison de pratiques obstétricales différentes. La fréquence et le type d'épisiotomies varient d'un pays et d'un centre à l'autre.

En général, les taux de lésions périnéales sévères sont beaucoup plus élevés aux Etats-Unis (où l'épisiotomie médiane est largement pratiquée) qu'en Europe.

En 2004, une étude issue d'un registre américain $(n=375$ 351) retrouvait respectivement $3,7 \%, 9,6 \%$ et $12,5 \%$ de déchirures du 3ème degré et $2 \%, 6,2 \%$ et $9,7 \%$ de déchirures du $4{ }^{\text {ème }}$ degré après un accouchement voie basse spontanée, une ventouse et un forceps [32] (NP3).

Les conditions de l'application instrumentale intervenant dans le risque de lésions périnéales sévères sont nombreuses :

- application à la partie haute surtout si elle est associée à une rotation de plus de $45^{\circ}$ (grade B),

- variétés postérieures (grade B),

- macrosomie fœtale.

Les RPC de 2006 ne concluaient pas à l'avantage de l'épisiotomie systématique.

Néanmoins, la réalisation d'une épisiotomie médiolatérale réduirait le risque de déchirure sphinctérienne en cas de ventouse (OR 0,11; IC 95\% 0,009-0,13) et de forceps (OR 0,08 ; IC 95\% 0,07-0,11) [33] (grade C). Elle doit être étudiée au cas par cas.

L'usage séquentiel de 2 instruments traduit une extraction difficile et multiplie les risques de déchirures périnéales sévères par un facteur 3 en cas de primiparité et de 9 à 11 en cas de multiparité par rapport à la voie basse spontanée ou à l'extraction réalisée avec un seul instrument [34] (grade C). 


\section{$>$ Complications tardives et à long terme}

Les risques d'incontinence anale dans l'année qui suit un accouchement par voie basse semblent être associés à la pratique d'une extraction instrumentale, notamment par forceps (1,4; IC 95\% 1,0-2,1) [35] (grade B).

Il n'existe qu'une seule étude ayant évalué le risque d'incontinence anale après une extraction par spatules [36]. Celle-ci retrouvait chez 159 primipares $24,5 \%$ de troubles de la continence anale dans un délai moyen de 27 mois après l'accouchement.

Le risque de lésions occultes du sphincter anal ne semble pas différent entre le forceps et la ventouse (grade B). L'incontinence anale persistante à distance d'une EI a une prévalence similaire quel que soit le mode d'accouchement, césarienne ou non, extraction instrumentale ou non, suggérant l'implication d'autres facteurs (grade B). Dans une étude randomisée anglaise $(\mathrm{n}=228)$, les pertes du contrôle sphinctérien anal étaient aussi fréquentes cinq ans après un forceps et une ventouse (15 versus $26 \%)$ [37].

L'EI ne semble pas être associée à une augmentation du risque d'incontinence urinaire persistante du post-partum par rapport à la voie vaginale spontanée (grade B). La prévalence globale de l'incontinence urinaire et de celle survenant à l'effort augmentent significativement entre les nullipares, les femmes ayant eu une césarienne et celles ayant accouché par voie vaginale [38].

L'EI ne semble pas être un facteur favorisant la survenue d'un prolapsus génital (grade C). L'impact de l'accouchement sur les risques de prolapsus génital est difficilement évaluable en raison des nombreux facteurs de confusion associés. Dans quatre études de cohortes, l'antécédent d'EI par forceps ou ventouse n'était pas associé significativement aux risques de prolapsus génital symptomatique [39].

L'EI et la présence de déchirures périnéales sévères majorent les risques de douleurs périnéales, de dyspareunie et de troubles sexuels du post-partum par rapport à la voie basse spontanée (grade C) [40,41]. 
Il semble qu'une extraction instrumentale difficile puisse être à l'origine de séquelles psychologiques pouvant faire renoncer à une autre grossesse (grade C) [42].

\section{b) Les mécanismes et facteurs incriminés des déchirures périnéales : Rappels anatomiques et Classification.}

\section{> Rappels Anatomiques [43,44]}

Le plancher pelvien :

Est constitué par l'ensemble des structures situées entre le péritoine pelvien et le plan de la vulve. Il constitue un hamac musculotendineux étalé transversalement dans le petit bassin qu'il divise en deux parties : le périnée en dessous et le petit bassin au dessus. Il comporte des éléments musculaires tapissés sur leur face profonde par le fascia pelvien pariétal.

Les muscle du plancher pelvien :

Les éléments musculaires du plan profond sont constitués essentiellement par les muscles releveurs de l'anus et les muscles ischiococcygiens. Ils circonscrivent entre eux, sur la ligne médiane et en avant du rectum, la fente urogénitale (hiatus urogénital) que traversent le vagin et l'urètre et qui constitue une zone de faiblesse du diaphragme pelvien [Figure 2].

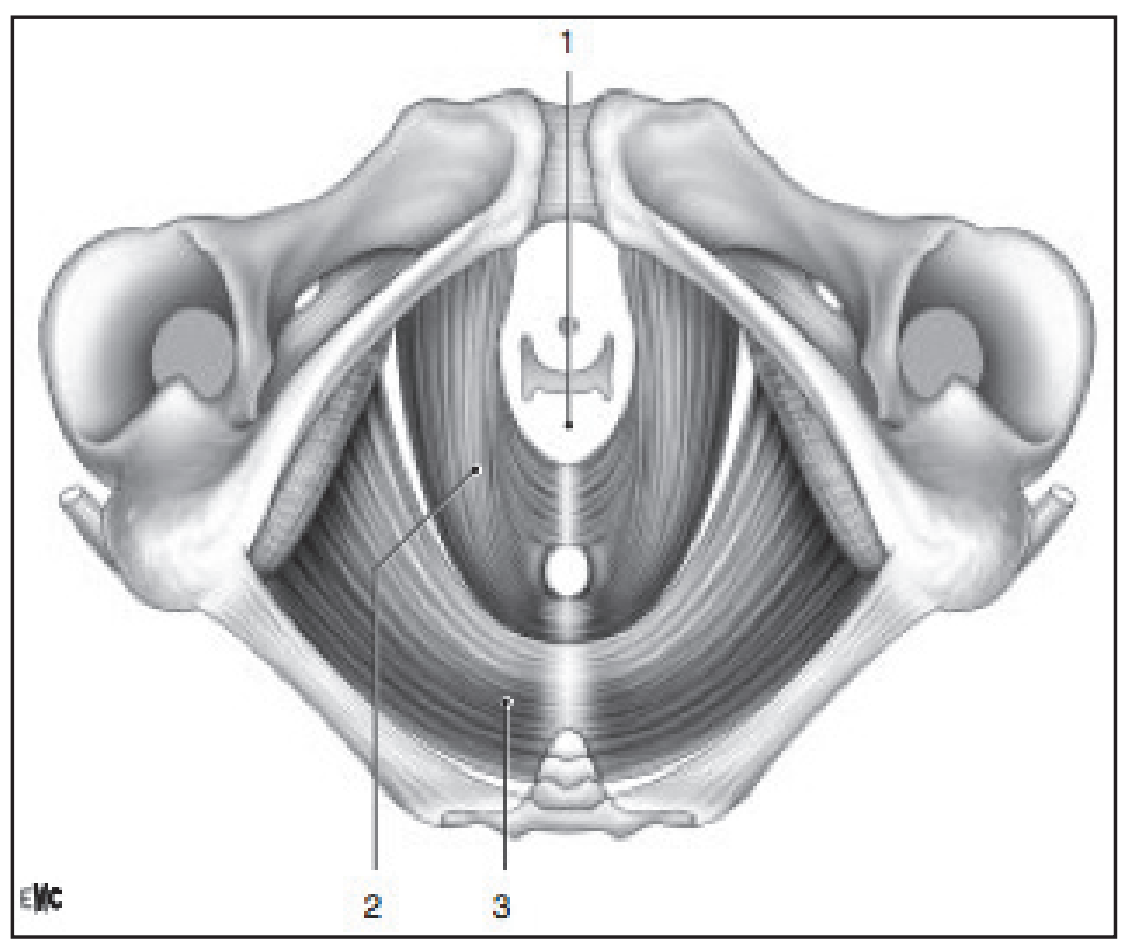

Figure 2 : Représentation schématique du diaphragme pelvien (vue inférieure).

1. Hiatus urogénital ; 2. Partie interne, élévatrice (faisceau lévatorien) ; 3. Partie externe, sphinctérienne (plaque des releveurs) : faisceau pubococygien, faisceau iliococcygien, faisceau ischiococcygien. 


\section{Le fascia pelvien pariétal :}

Il s'agit d'une formation aponévrotique qui cloisonne l'étage moyen et antérieur du périnée en tapissant la face profonde du diaphragme pelvien.

Le périnée:

Il correspond à l'ensemble des parties molles situées en-dessous du diaphragme pelvien. Sur le plan topographique, il est classique de distinguer le périnée urogénital (antérieur) situé en avant de la ligne tubéro-ischiatique et le périnée anal (postérieur) en arrière [Figure 3].

- Le périnée antérieur comporte deux plans :

- un plan superficiel encadrant le vestibule vulvaire, constitué de plusieurs muscles individualisés : le muscle constricteur de la vulve, le muscle bulbocaverneux, le muscle ischiocaverneux et le muscle transverse superficiel. C'est la partie la plus exposée aux déchirures obstétricales ;

- un plan plus profond engainé par le fascia pelvien pariétal comprenant l'appareil sphinctérien urétral, les formations érectiles (bulbes vestibulaires, corps caverneux) et le muscle périnéal moyen (anciennement transverse profond).

- Le périnée postérieur :

Il est centré par le canal anal et le sphincter externe de l'anus qui appartient au plan superficiel du périnée.

Le sphincter externe de l'anus est un manchon musculaire strié composé de deux couches entourant les trois quarts inférieurs du canal anal. Il s'insère en arrière sur le coccyx et le raphé anococcygien et en avant sur le centre tendineux du périnée. Il échange des fibres avec le faisceau puborectal du muscle releveur. Il entoure le sphincter interne et entre en contact direct avec la peau de la marge anale.

Le sphincter interne est constitué de fibres musculaires lisses orientées en bas et en avant et représente un renflement de la couche circulaire des muscles lisses de la paroi rectale.

De part et d'autre du canal anal, en-dessous du diaphragme musculaire pelvien, se situe l'espace ischiorectal. Il s'agit d'espaces conjonctivograisseux contenant un tissu celluleux, le paquet vasculonerveux pudendal, qui traverse le canal pudendal (anciennement canal d'Alcock).

Le périnée antérieur et le périnée postérieur sont solidaires sur le plan fonctionnel grâce au centre tendineux du périnée. Il s'agit d'une formation fibromusculaire, de section sagittale triangulaire, située sous la peau entre la partie inférieure du vagin et le canal anal qui prolonge le septum rectovaginal. Il donne insertion à de nombreux muscles périnéaux dont il constitue 
le point de convergence. Il joue un rôle essentiel dans la statique pelvienne et peut être lésé lors de l'accouchement.

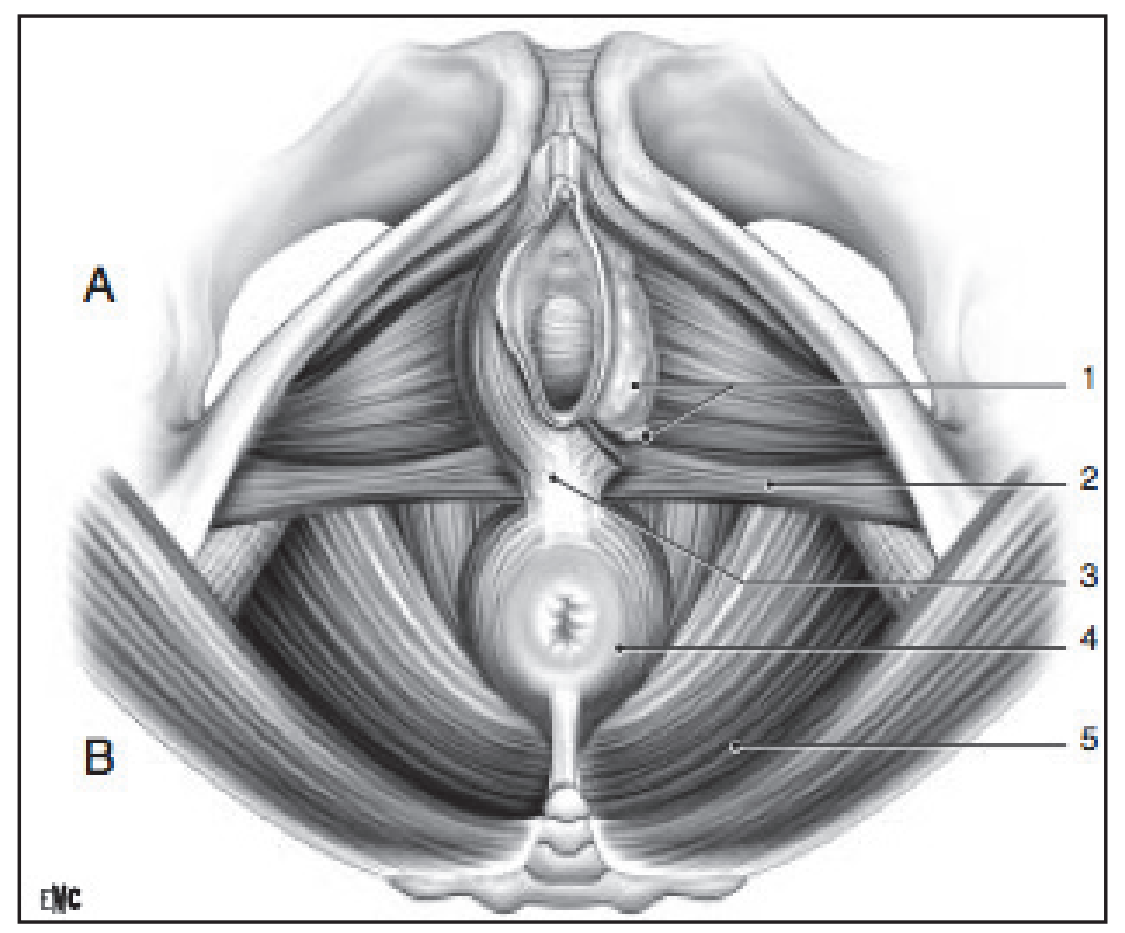

Figure 3 : Vue d'ensemble des muscles du périnée.

A. Périnée antérieur. B. périnée postérieur ; 1. Bulbe du vestibule et glande vestibulaire majeure ; 2. Muscle transverse superficiel ; 3. Centre tendineux du périnée ; 4. Sphincter externe de l'anus ; 5 . Muscle releveur de l'anus.

\section{Modifications périnéales au cours de l'accouchement}

Modification du segment anocccygien[43]:

$\mathrm{Au}$ début de sa descente, la présentation repousse le septum rectovaginal et aplatit le rectum contre le coccyx et le ligament anococcygien. La rétropulsion du coccyx entraîne un étirement de la commissure postérieure de l'anus et une saillie de celui-ci. Des lésions sphinctériennes anales souvent inapparentes peuvent apparaître à ce stade.

Ampliation du segment anovulvaire [44] [Figure 4] :

En descendant, la présentation se défléchit. Elle comprime et étale le centre tendineux du périnée, qui est laminé et distendu dans tous les sens. La région anovulvaire devient saillante (le périnée «bombe »). La distance anovulvaire s'allonge (triple ou quadruple) et le périnée s'amincit. Le plan de la vulve s'horizontalise. Le faisceau puborectal des releveurs, repoussé, vient s'intégrer dans le périnée superficiel. À ce stade, l'anus solidaire de la région anovulvaire 
s'ouvre largement et la muqueuse endoanale est visible. À ce stade, la distension est maximale et l'élongation provoque des lésions musculaires ou aponévrotiques quasi inéluctables. La rupture est imminente et l'épisiotomie doit être effectuée avant ce stade.

L'orifice vulvaire se déplisse et se distend pour laisser passer le mobile fœtal [44].

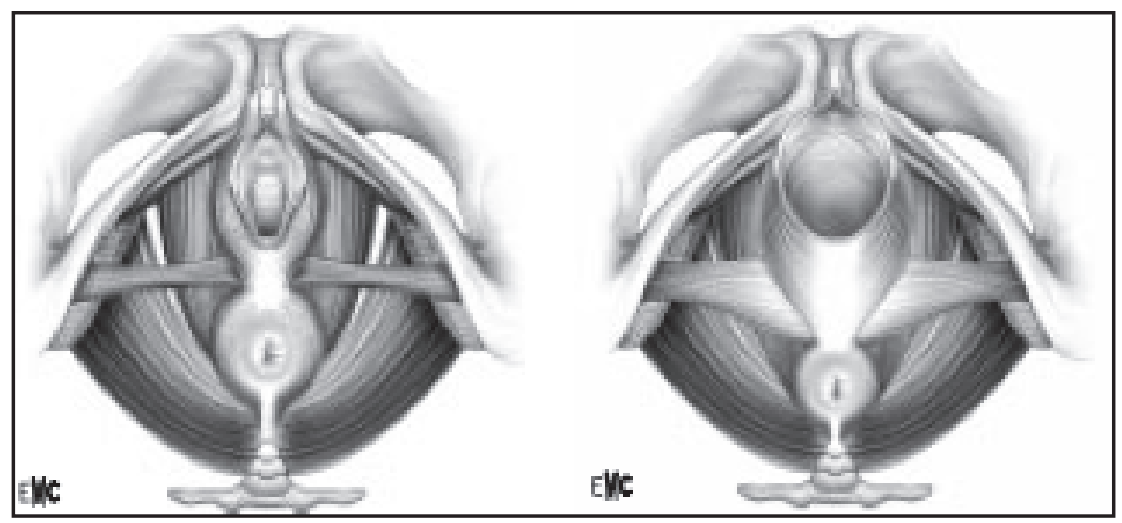

Figure 4 : Ampliation du périnée au cours de l'accouchement

\section{Les principaux mécanismes des lésions périnéales maternelles incriminés}

Il s'agit de l'augmentation des dimensions et de la distension excessive des parties molles par l'instrument et l'absence d'ampliation périnéale préalable liée à la rapidité de l'expulsion assistée. Elles regroupent schématiquement :

- les traumatismes de l'urètre et de son appareil sphinctérien :

L'élongation de l'urètre au cours de la grossesse est responsable d'une modification de l'angle urétrovésical postérieur qui tend à s'effacer. L'expulsion entraîne de façon constante une élongation du sphincter urétral. L'épisiotomie préventive, effectuée au bon moment, permet une prévention de ces lésions sphinctériennes et périurétrales [44].

- la désolidarisation interviscérale :

Lors de la descente de la tête, il peut se produire une désolidarisation interviscérale par un mécanisme de «piston ». Ce mécanisme entraîne des lésions des septa interviscéraux et des fascias [44].

- les lésions du plancher pelvien :

On peut observer des déchirures interstitielles lorsque le seuil d'élasticité des structures musculaires et aponévrotiques est dépassé. Le diaphragme pelvien distendu aura une contractilité affaiblie. Ces lésions peuvent intervenir sans déchirure périnéale apparente. Le 
centre tendineux du périnée, distendu par la présentation lors de l'ampliation du segment anovulvaire, peut être le siège de désinsertions terminales des fibres musculaires responsables des troubles de la statique pelvienne [44].

- les lésions neurologiques :

Il peut exister une neuropathie d'étirement du nerf pudendal en rapport avec la distension tissulaire de l'accouchement par voie basse [44].

\section{$>$ Classifications}

Il est important de rappeler les classifications française et de l'Organisation mondiale de la santé (OMS) des déchirures périnéales obstétricales, et leurs schémas anatomiques correspondants. [Tableau 4] [44,45].

Dans la suite de ce travail nous utiliserons la classification OMS. 
Tableau 4 : Classifications française et OMS des déchirures périnéales obstétricales et schémas correspondants.

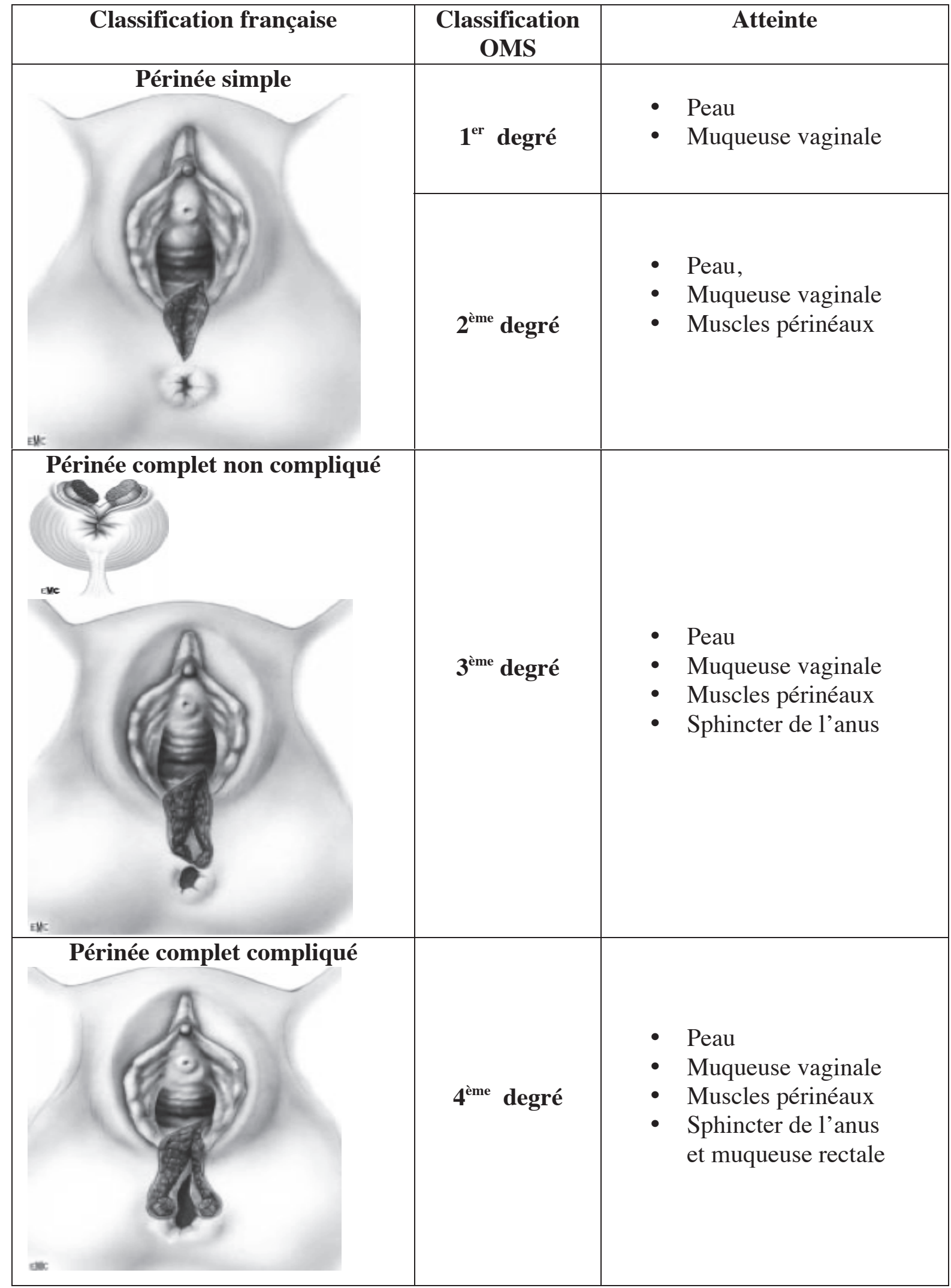




\section{Les déchirures périnéales du $1^{\text {er }}$ degré selon l'OMS}

Elles concernent la déchirure, la lacération ou la rupture du périnée intéressant la fourchette, les lèvres, la peau le vagin et la vulve, sans atteinte du fascia.

Les lésions vulvaires intéressent individuellement ou à la fois, les grandes lèvres, les petites lèvres (déchirures nymphéales), le clitoris (déchirures clitoridiennes) et l'hymen (déchirures hyménéales). Elles peuvent constituer de simples éraillures souvent non suturées, ou de véritables déchirures, sections, qui mériteront une réfection appliquée.

\section{Les déchirures du $2^{\text {ème }}$ degré selon l'OMS}

Elles concernent la déchirure, la lacération ou la rupture du périnée, intéressant les lésions du $1^{\text {er }}$ degré et les muscles du périnée du vagin ou plancher pelvien. Elles sont superficielles. La plaie est triangulaire, irrégulière et s'étend sur une des parois latérales du vagin, ou les deux ou en direction du sphincter anal. C'est la distension extrême des muscles péri-vaginaux et du plancher pelvi-périnéal par le mobile fœtal qui induit leur rupture. L'élargissement de la présentation par les cuillères du forceps ou des spatules, accentue bien cette surdistension. La lésion est compliquée par une atteinte du noyau fibreux central du périnée.

\section{$>$ Les déchirures du $3^{\text {ème }}$ degré selon l'OMS}

Elles sont caractérisées par l'atteinte du sphincter externe de l'anus qui s'ajoute aux lésions précédentes. L'espace pelvirectal est béant avec des lésions vaginales importantes. Elles représentent 1 à $2 \%$ des accouchements. Un toucher rectal est indispensable pour évaluer cette lésion sphinctérienne nécessitant un traitement spécifique et permettant l'évaluation d'éventuelles lésions concomitantes.

La déchirure du sphincter anal se produit au moment de l'ampliation maximale du périnée postérieur lors de la déflexion de la présentation ou du dégagement de l'épaule postérieure. La mise en place d'un instrument dans la filière génitale puis les forces de traction exercées peuvent exagérer la distension périnéale et favoriser la rupture tissulaire. La lésion du sphincter externe de l'anus peut être partielle, totale et associer le sphincter interne. Elle est habituellement localisée au niveau de sa partie antérolatérale droite, dans le prolongement d'une déchirure vaginale ou d'une épisiotomie médio latérale. Une lésion isolée du sphincter interne peut se produire à périnée intact et passer inaperçue. 
Le taux global de déchirure du sphincter anal après un accouchement varie de 0,1 à 10,2\% selon les auteurs [46].

Les principaux facteurs de risque significatifs de déchirure du sphincter anal retrouvés par les études de cohorte en analyse multivariée sont :

$\checkmark$ la primiparité [47-51].

$\checkmark$ la macrosomie (relation linéaire entre le poids de naissance et le risque périnéale sévère)

$\checkmark$ la dystocie des épaules [47,52-56].

$\checkmark$ les extractions instrumentales (forceps et ventouses).

$\checkmark$ les présentations en variétés postérieures [57,58].

$\checkmark$ la prolongation de la deuxième phase du travail [52-54, 59].

$\checkmark$ l'épisiotomie médiane [60,61].

$\checkmark$ D'autres facteurs sont également incriminés : l'obésité, certaines origines ethniques (indienne et asiatique), une distance ano-vulvaire courte $[47,53]$ et l'expression utérine [52].

Par contre le risque de déchirure périnéale sévère n'est pas modifié par la position d'accouchement [62]. A l'inverse, le contrôle manuel du dégagement de la tête fœtale réduit significativement le risque de périnée complet $[63,64]$.

\section{Les déchirures du $4^{\text {ème }}$ degré ou déchirure complète compliquée}

Elles se caractérisent par une atteinte de la muqueuse du canal anal.

Cette fois, en plus d'une lésion sphinctérienne, la déchirure vulvopérinéovaginale se complique d'une rupture de la paroi anorectale antérieure, créant un cloaque recto vaginal. Il s'agit d'une plaie digestive, à prendre en charge comme telle.

\section{c) Autres complications des extractions instrumentales}

\section{Les déchirures cervicales}

Elles sont exceptionnelles, souvent associées à des déchirures vaginales hautes voire à une rupture utérine ou parfois isolée. Leur prévalence est faible après une EI, variant de 0 à $3 \%$ 
[46] et sont liées à une pose précoce de l'instrument avant une dilatation complète. Dans $11 \%$ des cas, celles ci mesurent plus de $3 \mathrm{~cm}$.

\section{Les plaies urinaires}

Elles sont très rares en cas d'EI par ST. Elles surviennent majoritairement après une EI par forceps en l'absence d'une vidange vésicale ou des suites d'une ischémie détrusorienne par une compression de l'organe entre la présentation et le bassin osseux maternel. Cependant ce type de délabrement a pratiquement disparu avec les pratiques actuelles (restriction des grandes rotations de forceps au détroit supérieur) [46].

\section{Le thrombus génital}

Il s'agit d'une collection sanguine siégeant dans le vagin ou la vulve, le plus souvent secondaire à une rupture veineuse vulvo-vaginale. L'hémostase ne s'y faisant pas, cela engendre une étendue de l'hématome vers le haut, ou vers la base du ligament large puis dans le rétro péritoine. Il s'agit d'une complication rare de l'accouchement (moins d'un pour mille) $[65,66]$.L'EI est un facteur de risque classique au même titre que la primiparité, une déchirure d'un cul de sac vaginal et le poids naissance élevé [66].

\section{Hémorragie de la délivrance}

Plusieurs études de cohortes ont mis en évidence une augmentation significative du risque hémorragique après une EI par rapport à la voie basse spontanée (OR variant de 1,66 à 2,40) $[67,68]$. Les raisons invoquées sont l'augmentation des risques de déchirures des parties molles et d'atone utérine favorisée par la présence de facteurs associés (travail dystocique, $2^{\text {ème }}$ phase prolongée, macrosomie). Après extraction par ST, les pertes sanguines sont significativement plus élevées qu'après une voie basse spontanée (NP3)[8].

\section{2) Les complications néonatales des extractions instrumentales}

Il existe de nombreuses publications concernant les complications fœtales des EI.

Les RPC du CNGOF en 2008 les rappellent et en font une synthèse [11].

À côté des complications liées à l'extraction instrumentale proprement dite, le plus souvent bénignes, existent des complications plus sévères, impliquant la technique, mais aussi les évènements qui ont conduit à l'extraction, comme une dystocie ou une anoxie fœtale. Ces 
complications sont en général résolutives. Elles peuvent d'ailleurs s'observer dans un accouchement normal.

Il ne semble pas y avoir de séquelles neurocognitives induites par les instruments au long terme (grade B).

\section{Complications liées à la ventouse}

La survenue des céphalhématomes, est observée dans 12\% des accouchements assistés par ventouse (grade B), plus fréquemment que lors des accouchements par forceps $(6,5 \%)$ et spatules [4].

D’autres lésions plus rares ont été observées tels que les hématomes sous-cutanés diffus du cuir chevelu, des hémorragies rétiniennes, des hémorragies intracrâniennes ou paralysie du nerf facial. La fréquence respective de ces complications est peut-être sous-évaluée.

Les céphalhématomes sont de bons pronostics à court terme, contrairement à l'hématome sous-cutané diffus, situation rare, qui peut mettre en jeu le pronostic néonatal.

\section{$>$ Complications des extractions par forceps}

La fracture du crâne avec risque d'embarrure, rare, est assez spécifique de l'utilisation des forceps (grade B). Dans le réseau Aurore, une seule fracture sur 4589 naissances a été rapportée [69]. Elle est en général asymptomatique.

\section{Complications des extractions par spatules de Thierry}

Ces RPC ne mentionnent aucune complication néonatale attribuable aux EI par ST au vue de la rareté de celle-ci rapportée dans la littérature [1,2]. 


\section{C - LES SPATULES DE THIERRY}

\section{1) Description de l'instrument}

Il y a maintenant 63 ans, Dr E. Thierry présentait dans Presse Médicale un nouvel instrument d'extraction obstétrical, qu'il appelait « extracteurs » ou «spatules » [Figure 5] [70].

Ce sont deux leviers indépendants et symétriques, terminés par les spatules proprement dites.

Les spatules ne présentent ni articulation, ni vis de serrage, ni perforation pour passage de lacs. Il en existe deux tailles. Les spatules « classiques » sont les longues : elles mesurent 40 $\mathrm{cm}$, pèsent chacune $365 \mathrm{~g}$; les cuillers mesurent $16 \mathrm{~cm}$ de long sur $6 \mathrm{~cm}$ de large.

Il existe un 2ème type de spatules: les spatules de Tessier. Plus courtes, elles ont un maniement et des propriétés proches des ST, dont elles constituent une variante [71].

Elles ne feront pas l'objet de notre analyse.

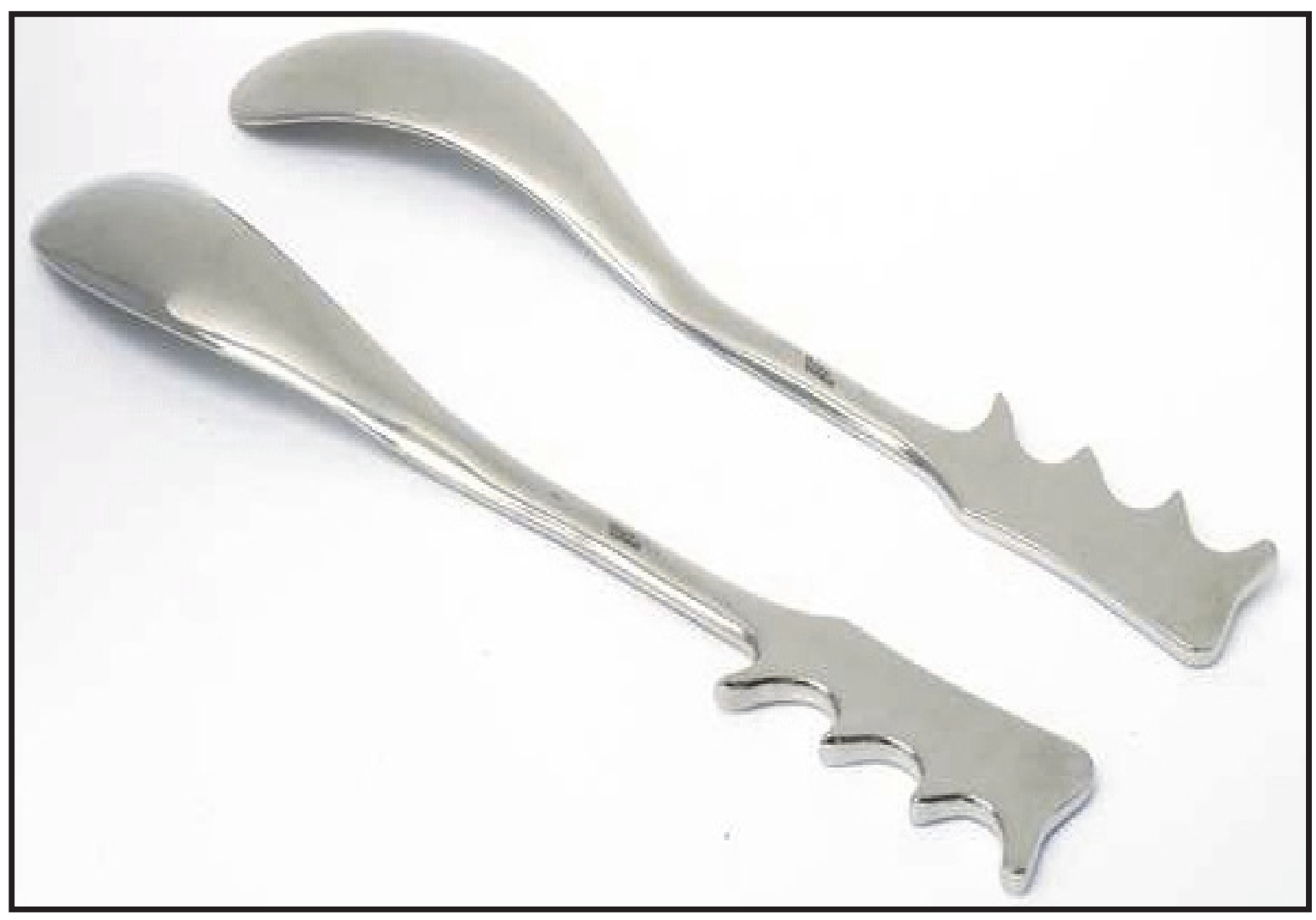

Figure 5 : Les spatules de Thierry. 


\section{2) Principes}

Les ST sont un instrument de propulsion et d'orientation de la tête fœetale, constitué de deux leviers symétriques et indépendants prenants appuis par l'extrémité de leurs faces internes sur la région malaire fœetale et par leurs faces externes sur le périnée maternel qu'elles écartent. Les ST ont été considérées par son inventeur comme la «prolongation des mains de l'accoucheur » [72].

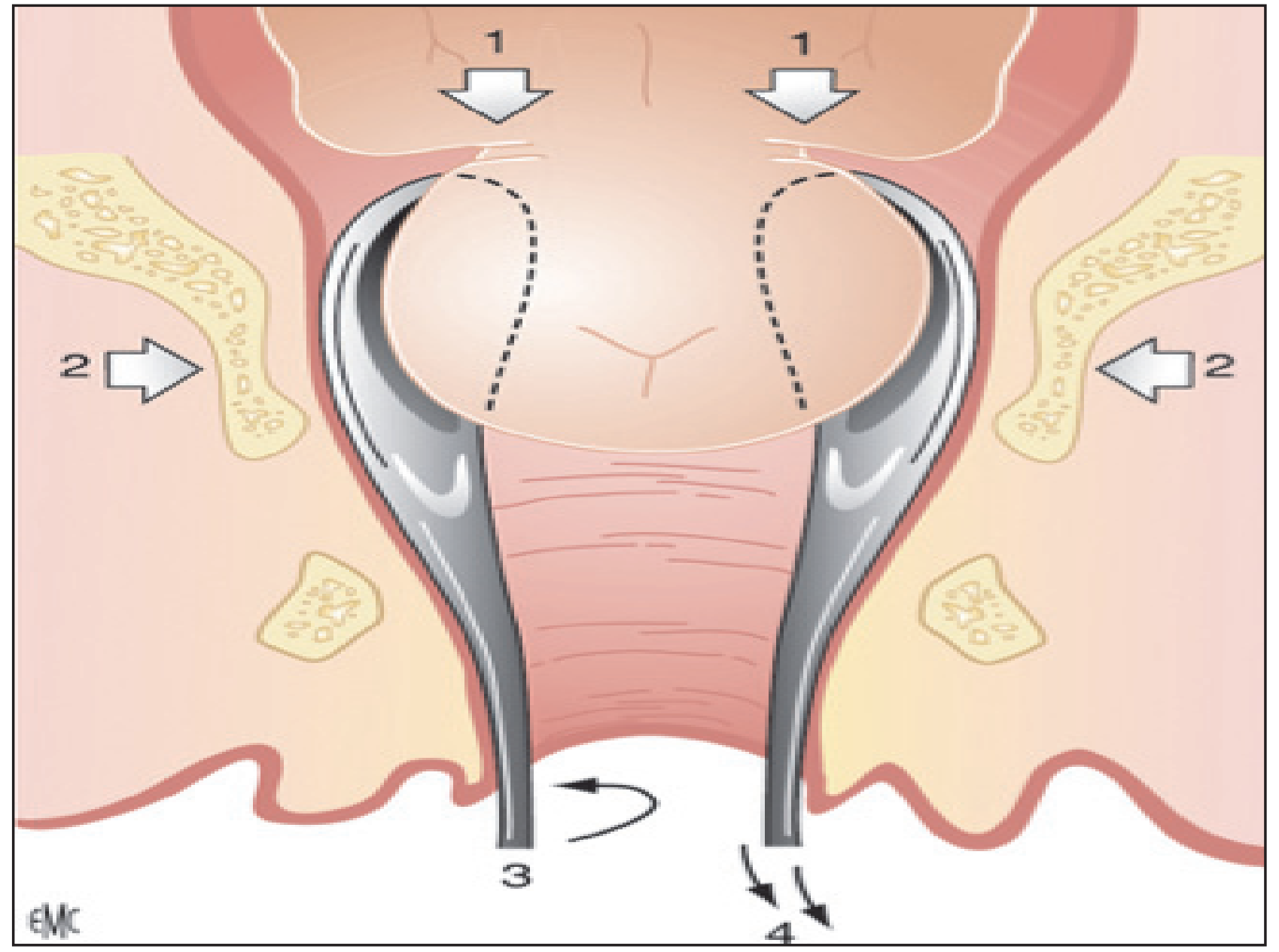

Figure 6 : Principes d'action des spatules. 1. Point d'appui distal sur l'os malaire ; 2. Point d'appui pelvien ; 3. Suppression de la résistance pelvienne ; 4. La force de traction s'exerce au niveau des manches. 
Le point d'application du levier se fait spontanément au tiers moyen de la face convexe des spatules. Dès que la traction commence en écartant légèrement les manches, l'extrémité des spatules propulse la présentation en même temps que la résistance des tissus maternels est diminuée. Est ainsi associée à la force de traction, qui reste réduite, une force de propulsion due à un effet de levier. Cette force explique la rareté des «dérapages » de l'instrument lors d'une utilisation correcte. L'appui du levier sur la face pelvienne n'est pas fixe, mais varie puisque les spatules suivent la progression de la tête [Figure 6]. L'extrémité fotale n'est pas tenue : son évolution entre les cuillers est absolument libre. Cette évolution se fait, le plus souvent, vers la réduction des diamètres, c'est-à-dire dans le sens d'une flexion et d'une rotation spontanée [72].

Les spatules sont donc un instrument de propulsion et d'orientation. À la différence du forceps, elles n'enserrent pas la présentation et ne sont pas considérées comme un instrument de traction. En revanche, l'écartement des tissus maternels n'est pas le fait de la présentation, mais des spatules elles-mêmes [72].

\section{3) Description du geste d'extraction}

\section{Mise en place de l'instrument}

Les spatules doivent être mises en place de manière symétrique et être, à la fin de la mise en place, parallèles à la suture sagittale. La spatule postérieure est mise en place la première. Quand la tête est dans le diamètre oblique gauche, comme dans la variété en occipito-iliaque gauche antérieur (OIGA) ou en occipito-iliaque droit postérieur (OIDP), le pariétal postérieur est du côté droit de l'opérateur ; la spatule droite est introduite la première ; elle est, pour l'introduction, tenue par la main gauche. Si la tête est dans le diamètre oblique droit (variété en occipito-iliaque droit antérieur (OIDA)) ou en occipito-iliaque gauche postérieur (OIGP), le pariétal postérieur est du côté gauche de l'opérateur, c'est la spatule gauche qui est introduite la première ; le talon du manche est tenu par la main droite [72].

La spatule est présentée parallèlement au bord postérieur de la vulve, et est poussée doucement au contact de la tête fœtale, cependant que la main controlatérale écarte les parties molles et maintient le bec de la spatule au contact de la présentation : le bec de la spatule ne 
doit jamais perdre le contact avec la tête fœtale (de manière à éviter de pincer un repli vaginal). Si l'on constate une difficulté à pousser la spatule, il peut exister un repli vaginal ou surtout cervical méconnu et l'indication doit être réévaluée [72].

Une fois que la spatule est suffisamment introduite (amenée presque à l'horizontale), commence le tour de spire de Madame de Lachapelle, qui est ainsi plus ample et plus décomposé que dans la mise en place du forceps. Ce tour de spire amène la spatule parallèle à la grande suture [72].

On change de main et l'on met en place la spatule controlatérale, de la même manière. La mise en place demande une grande décomposition des gestes, et un tour de spire de bonne amplitude. Cette manière de mettre en place les spatules avec un tour de spire ample fait que la cuiller contourne l'épine sciatique. Une fois en place, les cuillers prennent la face fœtale. Leurs axes correspondent à l'axe occipito-mentonnier [Figure 7] [72].

Les manches et les poignées doivent être bien parallèles, symétriques par rapport à la structure sagittale [72].

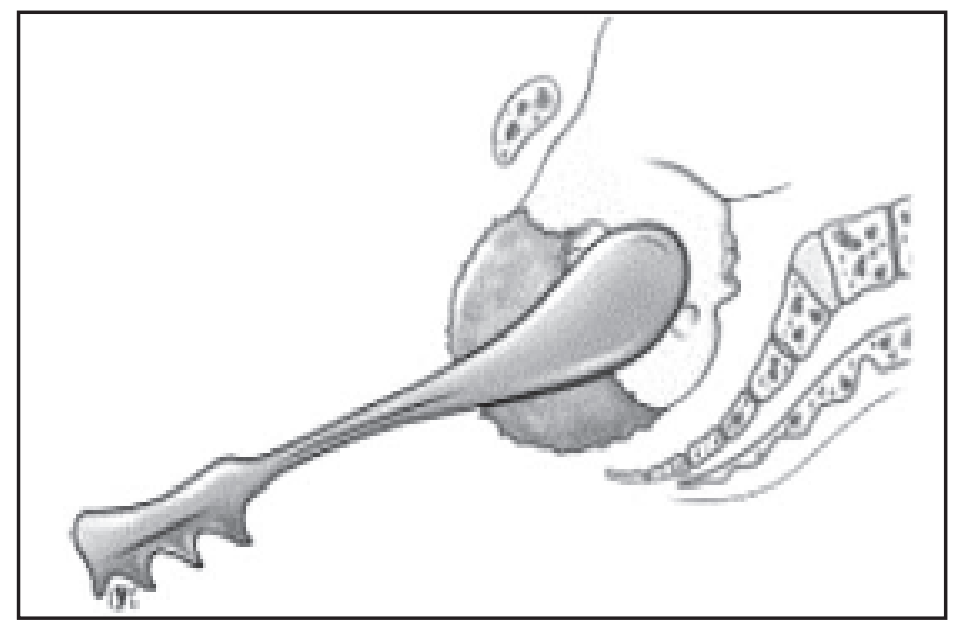

Figure 7 : Spatules en place selon l'axe sous-occipito-mentonnier. 


\section{Traction}

Les tractions peuvent alors commencer, la spatule droite prolongeant la main droite de l'accoucheur, la spatule gauche prolongeant la main gauche (« spatule droite = main droite, spatule gauche $=$ main gauche $»)$. Les tractions sont contemporaines des effets expulsifs maternels, si ceux-ci sont conservés, ou contemporaines des contractions. On combine un mouvement modéré d'écartement des poignées avec une traction douce. L'axe global des manches doit se situer dans l'axe de l'excavation : pour les prises hautes, l'axe de traction est très oblique en bas. Dès cette traction et l'écartement des poignées, les spatules ne peuvent fuir vers l'extérieur, mais elles entrent en contact avec la paroi pelvienne. L'effet de levier apparaît alors [Figure 8] [72].

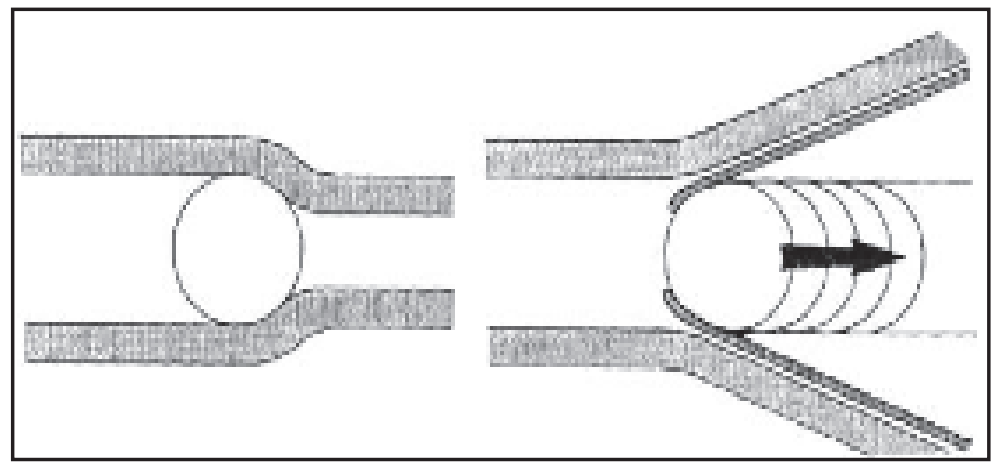

Figure 8 : L'effet levier des spatules.

Les ST constituent un instrument de propulsion. La force de traction appliquée sur le manche est transformée, par l'intermédiaire du point d'appui pelvien, en une force de propulsion de la tête fœtale.

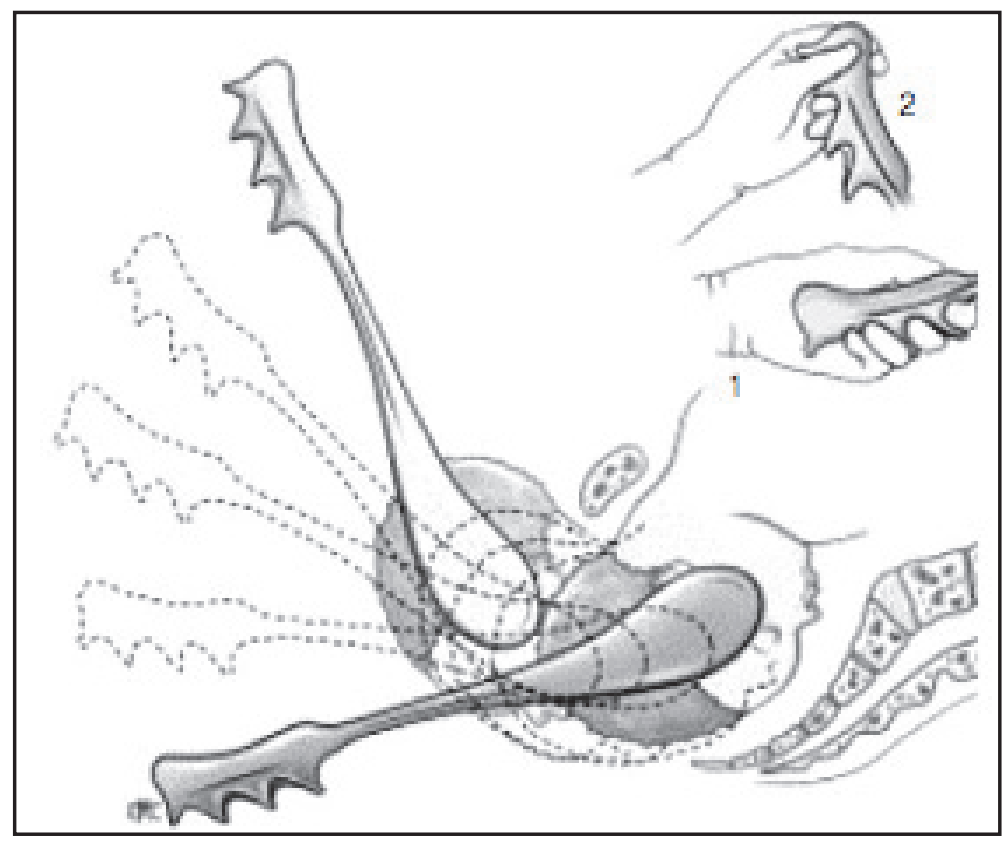

Figure 9 : Modification de l'axe des spatules lors de la progression de la présentation.

Initialement oblique vers le bas de manière à amener le sous-occiput sous la symphyse pubienne, cet axe se redresse avec la descente et la déflexion de la présentation. (1)Manière de tenir la spatule au cours de l'extraction ;(2) Manière de tenir la spatule pour la retirer avant le dégagement complet 
L'axe de traction suit l'axe de la progression du mobile foetal ; il se modifie avec la descente de la présentation, les manches, obliques vers le bas quand la tête est à la partie haute, se redressent progressivement vers l'horizontale cependant que la tête progresse .La traction peut aussi s'exercer par de petits mouvements alternés, qui ne doivent pas être des mouvements de va-et-vient, mais de progression vers la vulve, pour laisser la tête évoluer entre les spatules [Figure 9] [72].

\section{Le dégagement}

Pour assurer le dégagement, il faut descendre très bas la présentation, respecter au maximum la déflexion physiologique. Avec l'ampliation du périnée, les manches sont relevés, mais le relèvement doit être tardif, alors que le sous-occiput est bien calé sous le pubis, et les gestes doivent être effectués lentement [72].

On peut ensuite, très simplement, quand la tête distend bien l'anneau vulvaire, qu'il y ait ou non épisiotomie, guider la sortie de la tête en tenant les deux cuillers dans la même main ; des oscillations dans le sens transversal font progresser une bosse pariétale puis l'autre, cependant que la seconde main contrôle le périnée postérieur. Le contrôle total du dégagement du pôle céphalique permet une sortie «tempérée » de la tête fotale. Ce mode opératoire est préférable à celui qui, en écartant les spatules, écarte les tissus périnéaux et les expose aux déchirures : un écartement des spatules lors du dégagement est nocif pour le périnée [73,74]. Le périnée est moins allongé que dans les applications de forceps, car les spatules écartent les parties molles maternelles.

On peut aussi retirer les spatules, par un mouvement inverse de celui réalisé pour la mise en place ; il faut pour cela laisser le contact de la cuiller avec le crâne fœtal, par un mouvement horizontal amenant la spatule vers la cuisse maternelle. On termine ensuite l'accouchement normalement [Figure 9]. 


\section{4) Avantages et limites des spatules de Thierry}

Il n'existe pas d'étude publiée comparant les ST et les autres moyens d'extraction fœetale. Les comparaisons éventuelles entre les ST et autres instruments sont donc indirectes.

Leurs indications ainsi que leurs conditions d'application sont comparables à celles du forceps. Ce mode d'extraction n'a pas de contre indication spécifique (avis d'expert) [16].

Cet instrument paraît peu traumatique pour le fœtus (avis d'expert) [16]. Il est rapide à mettre en œuvre, efficace et permet de faire face des situations obstétricales plus ou moins critiques : rétention tête dernière, présentation de la face, prématurité (protection du crâne ?), variétés transverses. Leurs maniements peuvent être difficiles en de variétés postérieures, ou de présentation hautes et nécessitent un apprentissage spécifique.

Leur principal inconvénient est le recours fréquent à l'épisiotomie, notamment chez les primipares, mais sa réalisation ne doit pas être systématique (avis d'expert). [16] En effet, ce mode d'extraction peut avoir une connotation péjorative en raison du risque maternel vaginal et périnéal et de leur champ d'action prétendu limité, mais à l'inverse à la réputation d'être moins traumatiques que le forceps pour le fœtus. Le tableau 3 synthétise les avantages et les inconvénients des spatules. [Tableau 5] [16,72].

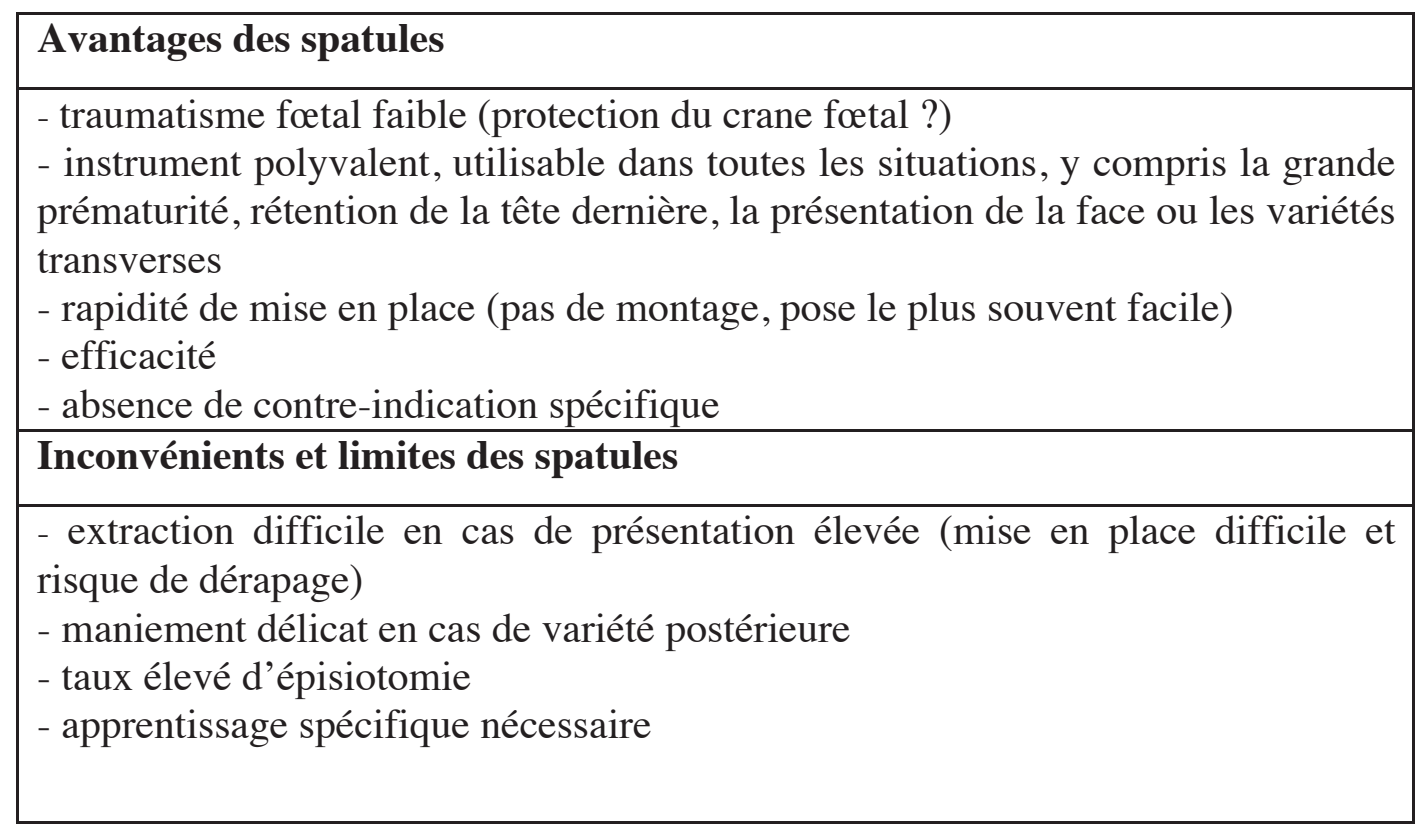

Tableau 5 : Synthèse des avantages et limites des spatules. 


\section{5) Complications des extractions par spatules de Thierry}

L'extraction par ST est considérée comme traumatisante pour le périnée maternel en raison de l'importance du volume, de l'absence de courbure pelvienne des branches et des mouvements d'écartements des parties molles maternelles lors de la propulsion du mobile fotale.

Dans une études rétrospective comparative incluant 279 extractions par ST, il existait une augmentation significative du risque de déchirure du $3^{\text {e }}$ degré par rapport à la vie basse spontanée (5,4 versus 0,7\%, OR 75 ; IC 95\% 1,5-32,3 )[8](NP4). Deux autres études rétrospectives françaises retrouvaient des taux de déchirures du $3^{\mathrm{e}}$ degré respectifs de 4 et $5 \%$ (NP4) [1,2]. Une étude rétrospective observationnelle qui avait été menée dans notre CHU sur 1065 d'extractions par ST retrouvait un taux de déchirures périnéales du 3ème et 4ème degré de 6,2\% [3]. Les facteurs de risque de déchirure du sphincter anal associés à l'extraction instrumentale sont la pose de l'instrument à la partie haute de l'excavation pelvienne, la présentation en variété postérieure et la macrosomie (NP2) [16].

Les spatules n'étant pas un instrument de flexion, leur utilisation en cas de variété postérieure peut majorer le risque périnéal par l'augmentation des diamètres céphaliques fœetaux et l'absence de rotation antérieure. Ainsi, une série rétrospective française de 163 extractions par spatules retrouvait un taux de lésions sphinctériennes anales plus important en cas de dégagement en occipito-sacré (OS) (17,4 versus 2,9\%, OR 6,46; IC 95\% 1,32-31,5) (NP4) [13]. Une autre série française de 42 extractions par spatules montrait respectivement 6,6 et $11 \%$ de déchirures du $3^{\text {e }}$ degré après une rotation instrumentale suivie d'un dégagement en occipito-pubien (OP) et en cas de variété postérieure persistantes (NP4)[14].

Il n'existe pas d'essai randomisé contrôlé comparant les ST à d'autres instruments d'extraction. Cependant une série française de 419 accouchements montrait un taux de lésion périnéale de second degré ou plus chez les patientes ayant accouché par voie basse, par spatules et par ventouse respectivement de 5,9\%,27\% et 28,6\%, ( $p<0,001)$. Si aucune différence significative n'a été observée entre le taux de lésions périnéales obtenues après extraction par spatules et ventouse, comparé aux accouchements par voie basse, seules les ST étaient associées à un risque significativement plus élevé de lésion périnéale de troisième degré ou plus : $2(0,9 \%)$ vs. $6(5,2 \%)$, respectivement $(p=0,022)$ [75]. Le respect des bonnes pratiques, la formation et l'expérience pourraient réduire les risques (NP2) [16]. 


\section{D - LES VENTOUSES}

\section{1) Description de l'instrument}

Toutes les ventouses présentent une cupule en métal ou en plastique, un système de traction et d'aspiration à l'origine d'une pression négative entre la cupule et la tête fotale. Il en existe plusieurs types dont la ventouse Kiwi ${ }^{\circledR}$

Cette dernière est utilisée au CHU Archet 2 de Nice [Figure 10].

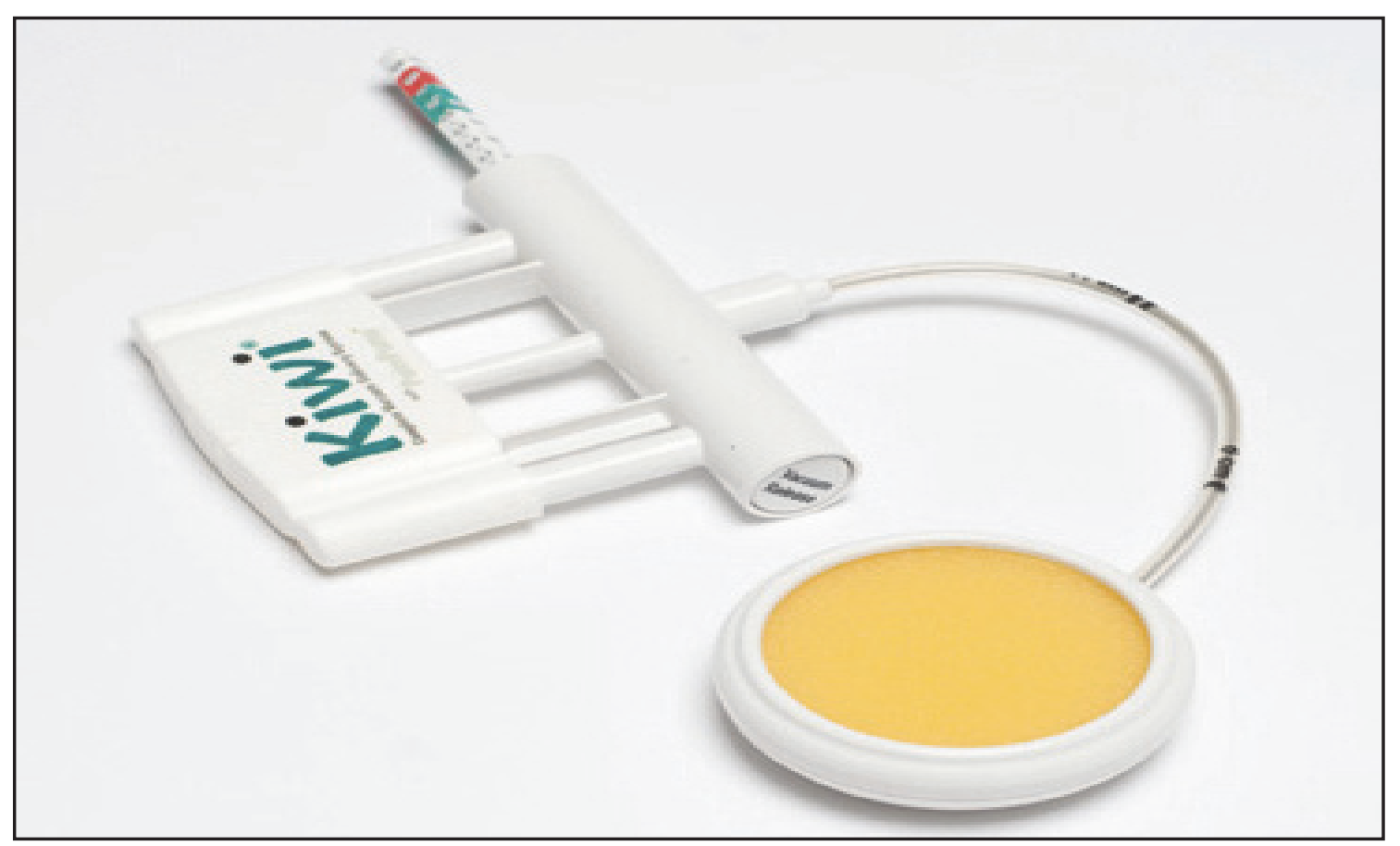

Figure 10 : La ventouse Kiwi ${ }^{\circledR}$.

\section{2) Principes}

La ventouse est un instrument de flexion céphalique, de traction et de rotation induite. Une dépression réglable est réalisée à l'intérieur de la cupule qui peut être métallique ou en plastique rigide. A leur sommet s'attache un fil ou une chaînette qui permet la traction. L'efficience de l'extraction fotale par ventouse est très dépendante du matériel utilisé et du respect du mode d'emploi [76]. 


\section{3) Description du geste d'extraction}

\section{Mise en place de l'instrument}

Une position correcte de la cupule sur la tête fœtale est garante du succès de la manœuvre. La cupule doit être appliquée sur le «point de flexion». Ce «point de flexion » est situé sur la suture sagittale 2 à $3 \mathrm{~cm}$ en avant de la petite fontanelle à l'intersection entre le diamètre sincipito-mentonnier et le scalp fœtal [Figure 11] [76].

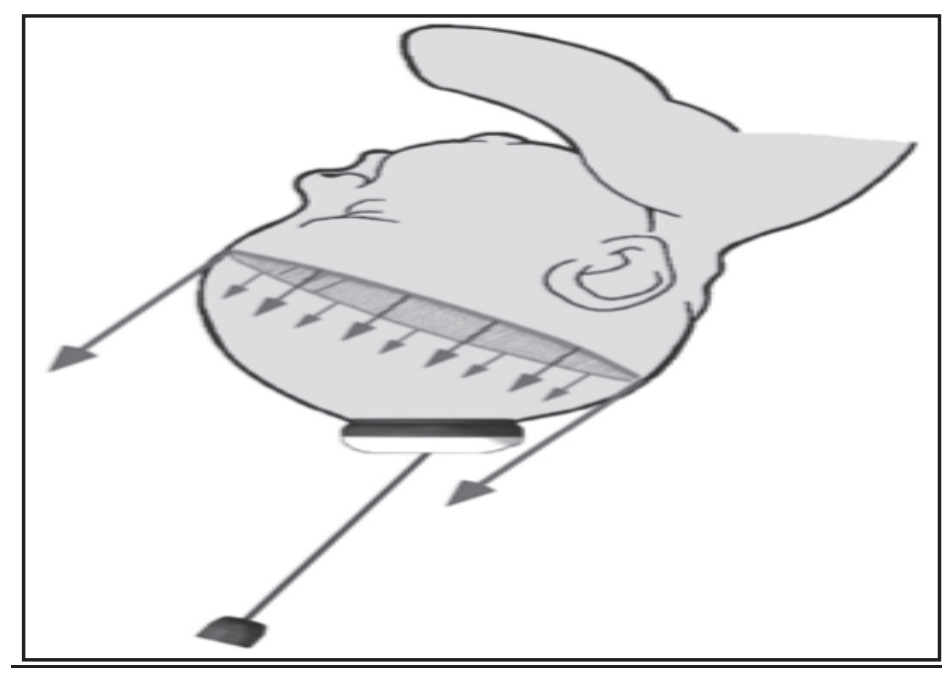

Figure 11 : Zone d'action de la ventouse obstétricale sur le cuir chevelu fotal

\section{Flexion}

Les tractions seront de faible intensité et continues. Tout d'abord, après repérage de la petite fontanelle, mise en place de la cupule sur le « point de flexion ». Ensuite, en fonction de la variété de position, six directions de tractions sont théoriquement possibles :

- OIGA : en bas et à droite

- OIDA : en bas et à gauche

- OIGT (Occipito iliaque gauche transverse) : horizontalement et à droite

- OIDT (Occipito iliaque droite transverse) : horizontalement et à gauche

- OIGP : en haut et à droite 
- OIDP : en haut et à gauche

Une fois la flexion maximale obtenue, il est parfois nécessaire de détacher la cupule pour la replacer le plus loin possible vers l'occiput fœetal avant d'entamer le second temps de la traction [76].

\section{Descente}

Ce second temps de la traction doit reproduire, aider sans contrarier, la progression naturelle du sommet dans la filière pelvienne. La traction ne s'effectue pas dans une direction unique, mais dans des directions différentes en fonction de la progression du sommet dans l'excavation.

A tous les niveaux de l'excavation pelvienne, les fils de traction doivent être perpendiculaires au plan de la cupule.

Au niveau du détroit supérieur, la traction est exercée dans l'axe ombilicococcygien.

Les lacs de traction de la ventouse au contact du coccyx dépriment le périnée postérieur [Figure 12] [76].

L'opérateur débute la traction en s'asseyant ou en s'agenouillant au ras du sol et tirer vers ses pieds.

Il relève ensuite la direction de la traction graduellement pour atteindre l'horizontale quand la cupule apparaît sur le périnée, et enfin, toujours en remontant progressivement la direction de la traction, arriver presque à la verticale au moment de l'expulsion.

Les efforts de traction, toujours modérés, sont effectués pendant les contractions utérines, aidés par la poussée abdominale maternelle ; entre deux contractions utérines, on se contente de maintenir le mobile fotal au niveau obtenu au moment de la dernière poussée.

L'engagement puis la descente du sommet sont contrôlés par les doigts de la main libre de l'opérateur qui restent intravaginaux [23]. 


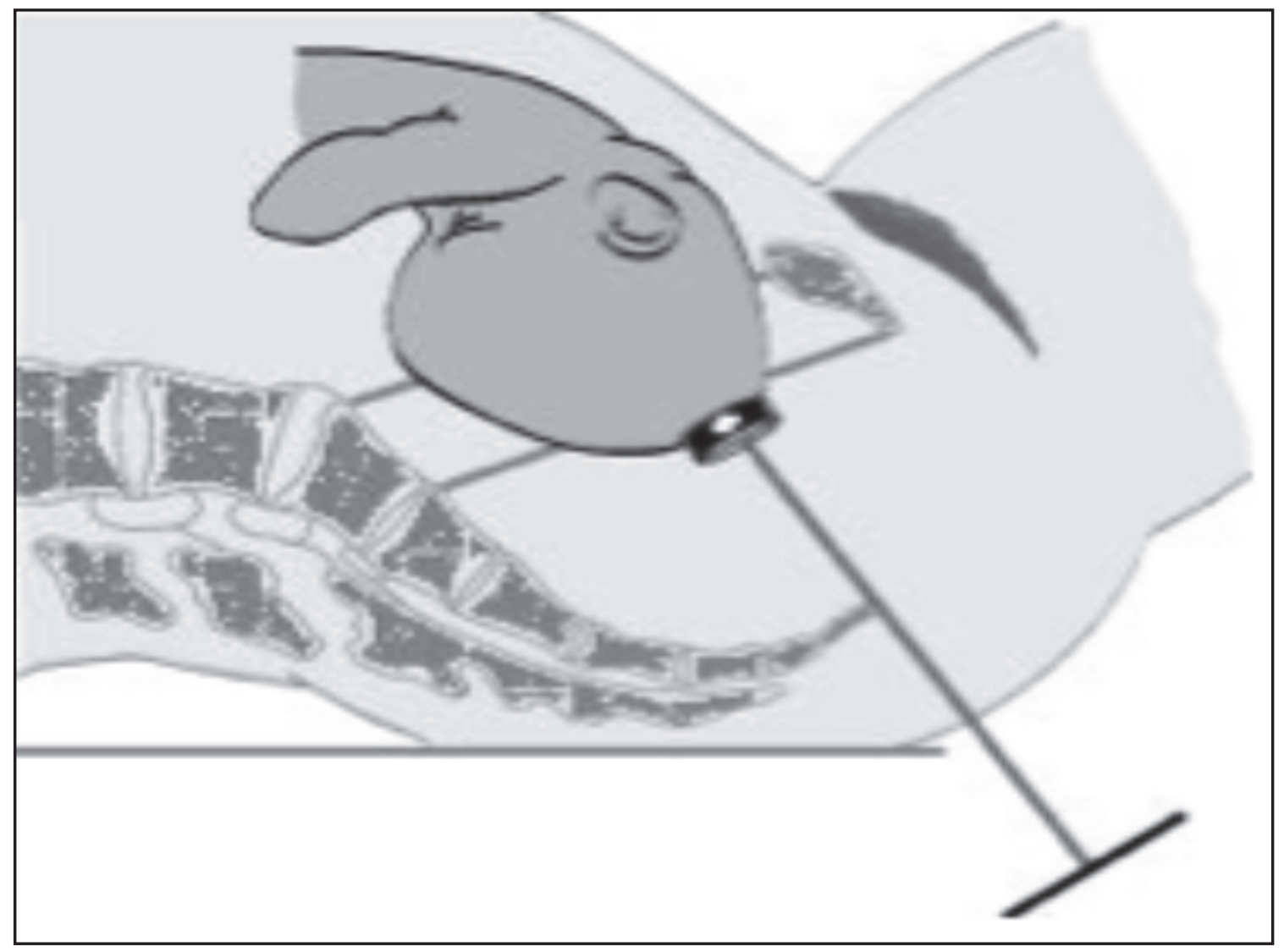

Figure 12 : Traction dans l'axe ombilicococcygien.

\section{4) Avantages et limites des ventouses obstétricales}

Il existe de nombreuses publications sur les avantages de cet instrument, le premier étant le risque inférieur de lésion périnéale sévère. De plus la ventouse permet d'induite une rotation dans l'accouchement des variétés postérieures. Enfin, ce mode d'extraction semble etre soumis à une courbe d'apprentissage plus courte que celle du forceps [16].

La durée de l'extraction est légèrement plus rapide avec le forceps qu'avec la ventouse (grade C) [16]. Cependant, le caractère urgent de l'extraction ne devrait pas être un argument pour choisir un instrument plutôt qu'un autre (accord professionnel) [16].

Les limites de cet instrument sont principalement le risque d'échec de la technique [77] et le risque de complications feotales [4]. 
La fréquence des échecs, difficilement estimable car non publiée, serait de $0,5 \%$ pour Sarapola et son équipe, entre $5 \%$ et $9 \%$ pour Edozien dont $85 \%$ d'échecs de ventouse et $1 \%$ d'échecs de forceps [77]. La durée d'application de la ventouse obstétricale doit être inférieure à 20 minutes (accord professionnel)[16]. L'absence de progression du mobile fœtal après 6 contractions utérines doit faire renoncer (accord professionnel) [16]. Trois lâchages correspondent à un échec et doivent faire renoncer (accord professionnel) [16]. Il n'y a pas d'argument dans la littérature pour recommander un forceps ou une césarienne après échec de ventouse. Le choix doit être adapté au cas par cas selon l'expérience de l'opérateur (accord professionnel) [11].

La ventouse souffre d'une mauvaise réputation concernant les complications fœtales. Les défenseurs de la ventouse pondèrent ce point en expliquant qu'il s'agit le plus souvent de troubles mineurs sans conséquence à long terme. La fréquence des complications les plus constatées tel que les céphalhématomes varie de 6 à $20 \%$ selon les études publiées [4, 78,79]. Les forces exercées par la ventouse sur le scalp fœtal seraient à l'origine des lésions secondaires. Quatre types de contraintes s'appliquent sur le crâne : une pression de succion négative, une force de traction, une force circulaire en cas de rotation et des forces de cisaillement. La conjonction de ces phénomènes explique les différentes lésions observées et décrites sur le fotus : bosses sérosanguines, excoriations du cuir chevelu, décollement cutané localisé, cephalhématome, hématome sous-cutané diffus du cuir chevelu, hémorragies cérébroméningées ou rétiniennes, voir des fractures du crâne. Ces complications graves demeurent cependant très rares [16]. 


\section{E - LES FORCEPS}

\section{1) Description et principes}

Le forceps est composé de cuillères, porteuses de courbure céphalique pour enserrer la présentation fœtale. Les cuillères se prolongent par des manches avec lesquels elles ont une angulation plus ou moins marquée : la courbure pelvienne. Les manches peuvent se croiser et alors s'articuler par leur entablure. Ils peuvent aussi être parallèles ou divergents et sont solidarisés par des barres de solidarisation. Le forceps est un instrument de préhension, de traction et de rotation mais il n'est pas adapté à la flexion [80].

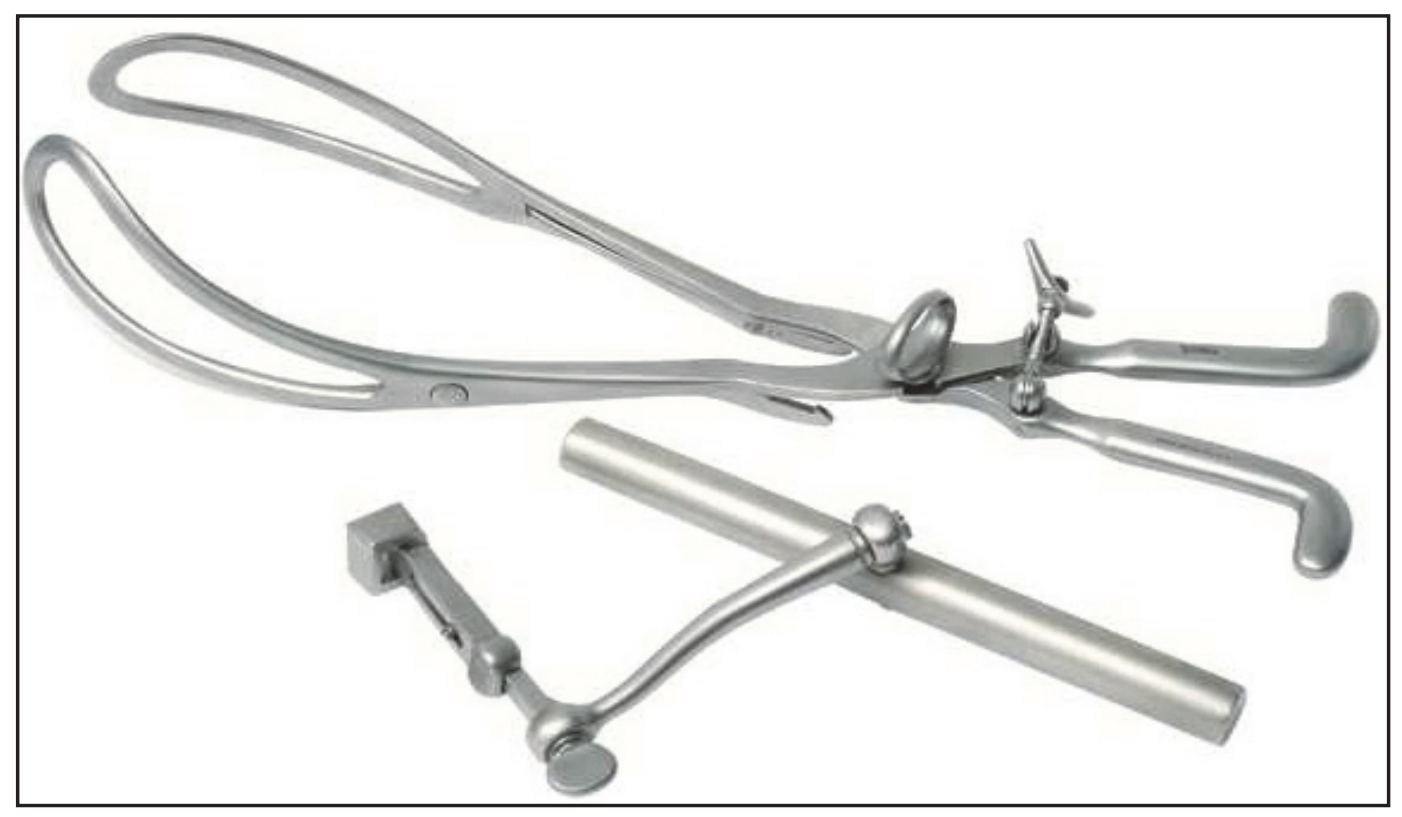

Figure 13 : Forceps de Tarnier

La formation au forceps en France repose sur différents modelés : Levret, Tarnier et Suzor. La formation à la pose de cet instrument est la même avec tous les différents types, en revanche la formation à la traction est plus délicate et diffère selon le type de forceps. Cidessous un modèle de forceps [Figure 13] [80]. 


\section{2) Avantages et limites des forceps}

Le forceps est un instrument irremplaçable, dont l'indication et la réalisation nécessitent une grande rigueur.

Les prises en variété transverse sont contre-indiquées avec un forceps aux branches croisées (accord professionnel) [16]. Il faudra alors plutôt utiliser un forceps à branches convergentes, ou de préférence un autre instrument (spatules ou ventouse) (accord professionnel) [16].

L'absence de progression du mobile fœtal après 3 tractions correspond à un échec de la technique (grade C) [16]. Le forceps s'accompagne de moins d'échecs d'extraction que la ventouse (grade $\mathrm{B}$ ). Les applications séquentielles sont très discutées. L'échec d'une application de forceps relève de la césarienne (accord professionnel) [16].

L'utilisation des forceps est associée à plus de déchirures périnéales sévères que celle des ventouses mais à moins de céphalhématomes et de dystocies des épaules [81].

L'utilisation des forceps est associée à plus de complications périnéales que celle des ventouses [81], incomplètement prévenues par la réalisation d'une épisiotomie.

Ce sont : [82]

- des plaies vaginales pouvant s'étendre jusqu'aux culs-de-sac vaginaux, avec un risque hémorragique ;

- des déchirures périnéales sévères ;

- exceptionnellement des plaies génito-urinaires qui peuvent se compliquer de fistules vésico-vaginales.

Le risque de complications néonatales est aussi redouté [80]. Fréquemment des éraillures et ecchymoses superficielles symétriques, sur le malaire sont observées, mais des complications rares et sévères ont été décrites telles que fracture du crâne, embarrure, paralysie faciale, et lésion cérébroméningée. En cas de prise irrégulière, la responsabilité du forceps peut le plus souvent être retenue. La fréquence de ce type de complications graves reste extrêmement faible dans les grandes séries, de 1 'ordre de 1,1\% [4] et l'utilisation des forceps est associée à moins de céphalhématomes et de dystocies des épaules que celle des ventouses [81]. 


\section{F - L'ODON DEVICE : UN NOUVEL INSTRUMENT D'EXTRACTION FOETALE}

L'Odon Device est un nouvel instrument d'accouchement vaginal assisté, simple et de faible coût, qui répond aux complications rencontrées lors d'une deuxième étape prolongée du travail d'accouchement, incluant potentiellement de fatales complications pour la mère (hémorragie, infection) et pour le nouveau-né (asphyxie à la naissance et traumatisme)[Figure 14] [83].

Cet instrument a été approuvé par l'OMS [83].

L'instrument est nommé ainsi en raison de son inventeur, un mécanicien argentin qui en a eu l'idée en étudiant une méthode pour extraire les bouchons des bouteilles vides.

Fabriqué en polyéthylène, son principe d'action est basé sur la diminution des forces de friction. Il est pour l'instant contre-indiqué en cas d'infection par le Virus de l'Immunodeficience Humaine.

Cet outil aurait de grands potentiels :

- les coûts de réalisation sont très bas pour le type de matériaux utilisé (cellophane et matières plastiques),

- la facilité d'utilisation : pourrait être utilisé même par un étudiant en lére année d'obstétrique,

- la fiabilité,

- En réduisant les contacts entre la présentation fotale et le canal pelvien, ce dispositif pourrait empêcher la survenue de certaines infections acquises pendant l'accouchement. 


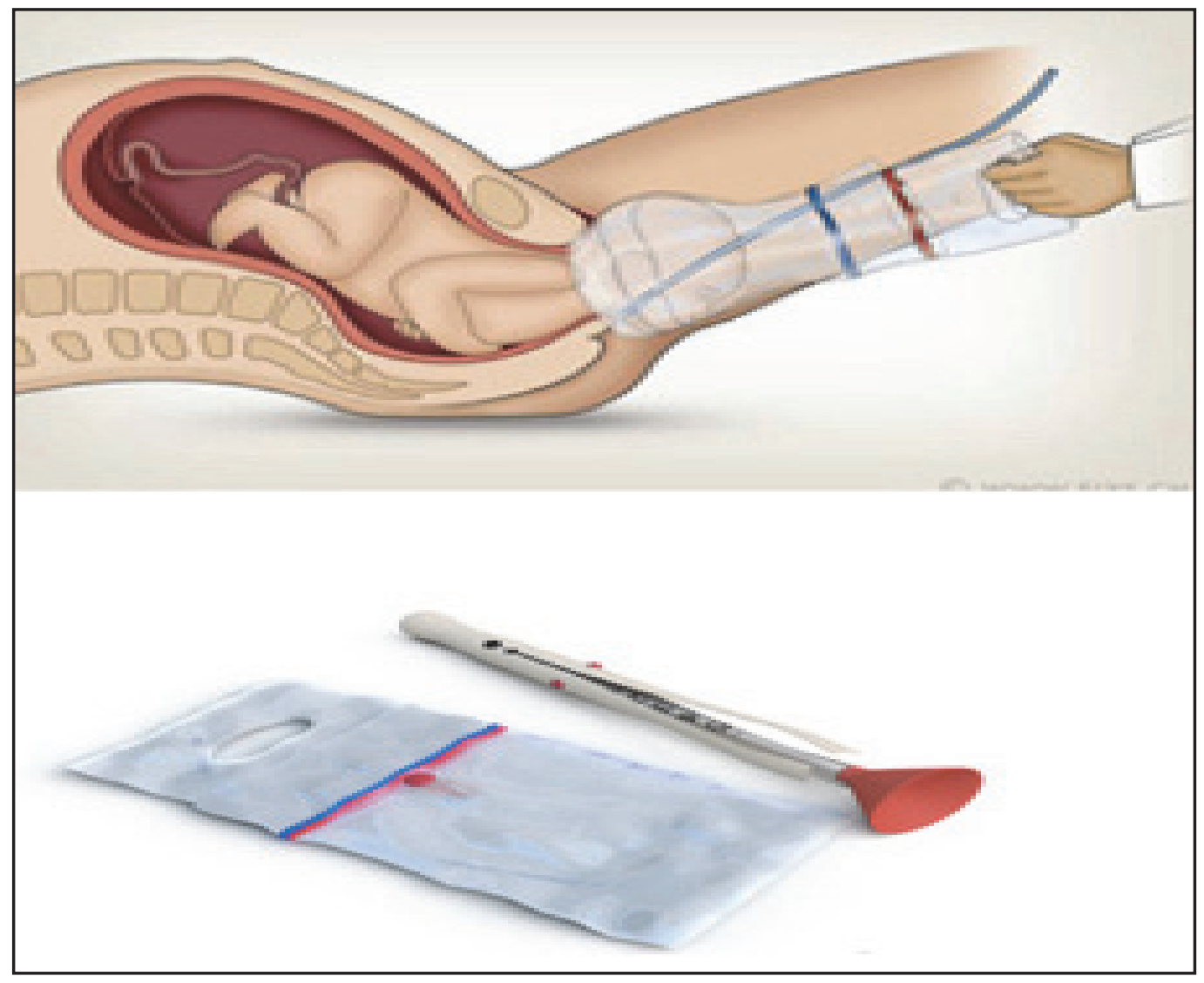

Figure 14 : Un nouvel instrument d'extraction : l'Odon Device.

A la fin de la première phase d'expérimentation sur 30 femmes, le Steering Comité se réunit à Monaco en novembre 2012 pour discuter de l'extension de l'étude à quatre autres centre : Argentine, Hong-Kong, Monaco, Suisse et définir le protocole de l'étude qui est approuvée.

La faisabilité, la sécurité et l'efficacité de cet instrument sont reconnus et permet la poursuite de l'étude et l'achèvement de la phase 1 (100 sujets supplémentaires) [Tableau 6].

Cet instrument serait plus sûr et plus facile à appliquer que les forceps et les ventouses. Il pourrait être une alternative intéressante en particulier dans les milieux où les ressources chirurgicales et humaines sont limitées [83].

Cet instrument ne fait actuellement l'objet d'aucune publication scientifique. 
Tableau 6 : Protocole d'étude du nouvel instrument d'extraction : l'Odon Device.

\begin{tabular}{|c|c|c|}
\hline BUT & OBJECTIF & PROTOCOLE D'ETUDE \\
\hline \multirow[b]{2}{*}{$\begin{array}{l}\text { Déterminer la faisabilité, la } \\
\text { sécurité et l'efficacité de } \\
\text { l'Odon Device en cas de } \\
\text { prolongement de la 2ème } \\
\text { phase du travail. }\end{array}$} & $\begin{array}{l}\text { Evaluer la faisabilité et la } \\
\text { sécurité de l'instrument } \\
\text { dans } \\
\text { les établissements de soins } \\
\text { de santé }\end{array}$ & $\begin{array}{l}\text { Phase } 1 \\
\text { Test sur } 100 \text { patientes dans les conditions } \\
\text { d'un accouchement normal en Argentine. } \\
\text { Test sur } 30 \text { patientes dans les conditions } \\
\text { d'un accouchement normal en milieu } \\
\text { rural d'Afrique du sud. }\end{array}$ \\
\hline & $\begin{array}{l}\text { Evaluation préliminaire de } \\
\text { son efficacité dans les } \\
\text { établissements de soins de } \\
\text { santé. }\end{array}$ & $\begin{array}{l}\text { Phase } 2 \\
\text { Randomisation de } 60 \text { patientes présentant } \\
\text { une 2ème phase du travail prolongée dans } \\
\text { le groupe d'extraction standard ou le } \\
\text { groupe d'extraction par l'Odon Device en } \\
\text { Argentine. } \\
\text { Randomisation } 60 \text { patientes présentant } \\
\text { une 2ème phase du travail prolongée dans } \\
\text { le groupe d'extraction standard ou le } \\
\text { groupe d'extraction par l'Odon Device en } \\
\text { Afrique du sud. }\end{array}$ \\
\hline
\end{tabular}




\section{G - EPIDEMIOLOGIE : QUELS INTRUMENT UTILISE-T-ON EN FRANCE?}

1) Distribution géographique du taux d'extraction instrumentale en CHU de niveau III en 2007

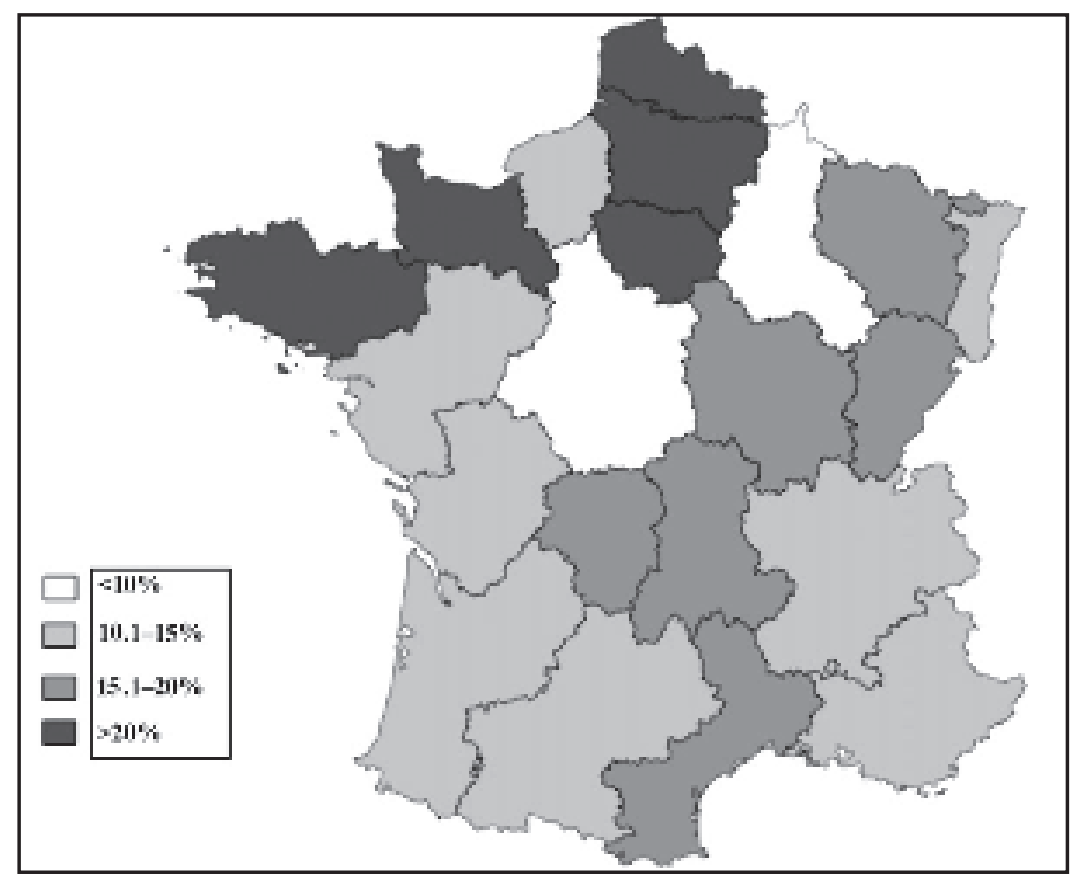

Figure 15 : Répartition géographique régionale des taux d'extractions instrumentales en CHU de niveau III en 2007

Alors que les RPC [16] en France ont été publiées en décembre 2008, une enquête nationale auprès de l'ensemble des CHU de France a été réalisée pour connaître la répartition des extractions instrumentales : elles sont pratiqués en CHU à un taux moyen national de 16,9\% des accouchements voies basses, ce qui est comparable avec les chiffres des pays voisins, tels que ceux du Royaume-Uni qui varient de 12 à 15\% [84]. Ce taux était de 11\% en France pour l'ensemble des maternités selon le réseaux AUDIPOG (Association des Utilisateurs de Dossiers Informatisé en Pédiatrie, Obstétrique et Gynécologie) [23]. On note cependant une grande disparité de ce taux d'extraction instrumentale sur le territoire allant de 5,3\% à 34,1\% d'un centre à l'autre [Figure 15]. Il existe aussi une disparité géographique du type d'instrument d'extraction utilisé [Figure 16, 17 et 18]. Cependant, la notion d'Ecole obstétricale n'est plus aussi soulignée que dans les 3 dernières décennies car la plupart des 
obstétriciens en formation apprennent la maitrise d'au moins deux instruments différents $[84,85]$.

2) Distribution géographique régionale de la part des spatules dans l'extraction instrumentale en CHU de niveau III en 2007

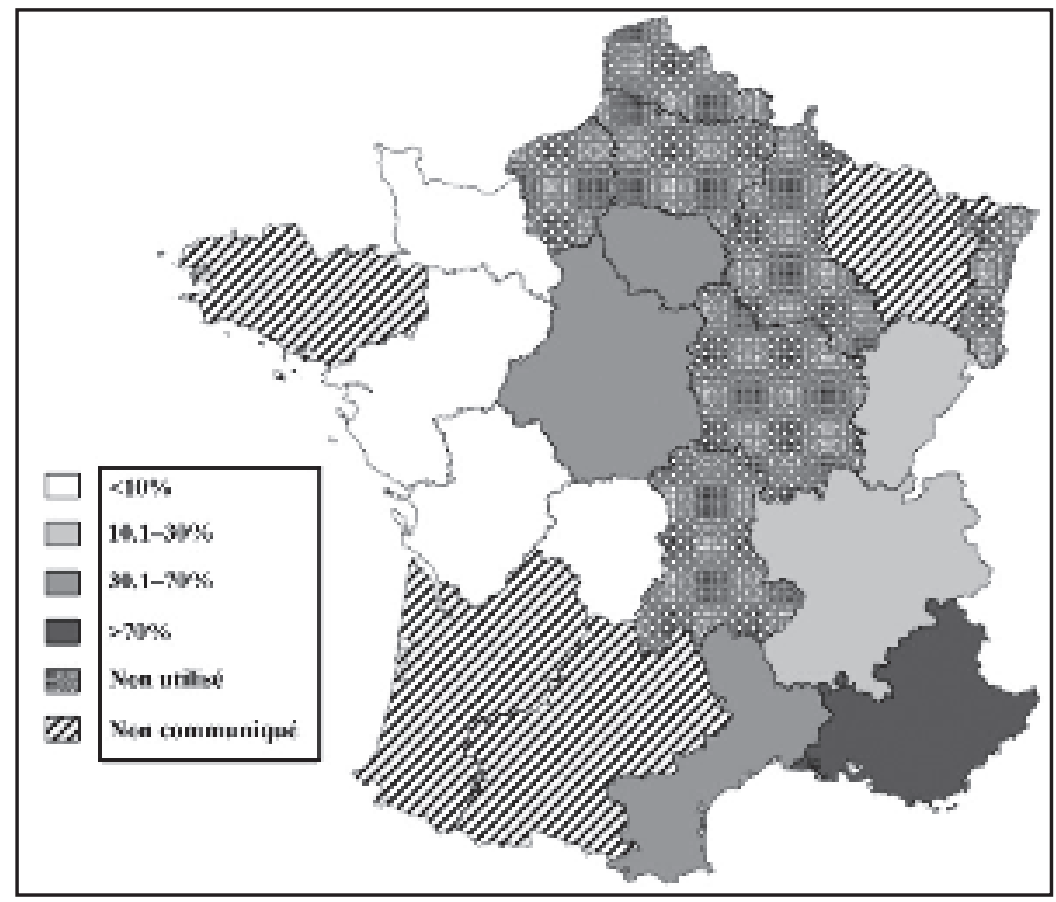

Figure 16 : Distribution géographique régionale de la part des spatules dans l'extraction instrumentale en CHU de niveau III en 2007

Les spatules sont peu utilisées en France avec un particularisme régional et totalement méconnues de nombreux pays : son utilisation occupe le premier rang dans les CHU du Sud de la France. Cet instrument a sa place dans l'arsenal thérapeutique obstétrical actuel et doit continuer à être enseigné (avis d'expert) [Figure 16] [16,84]. 
3) Distribution géographique régionale de la part des forceps dans l'extraction instrumentale en CHU de niveau III en 2007.

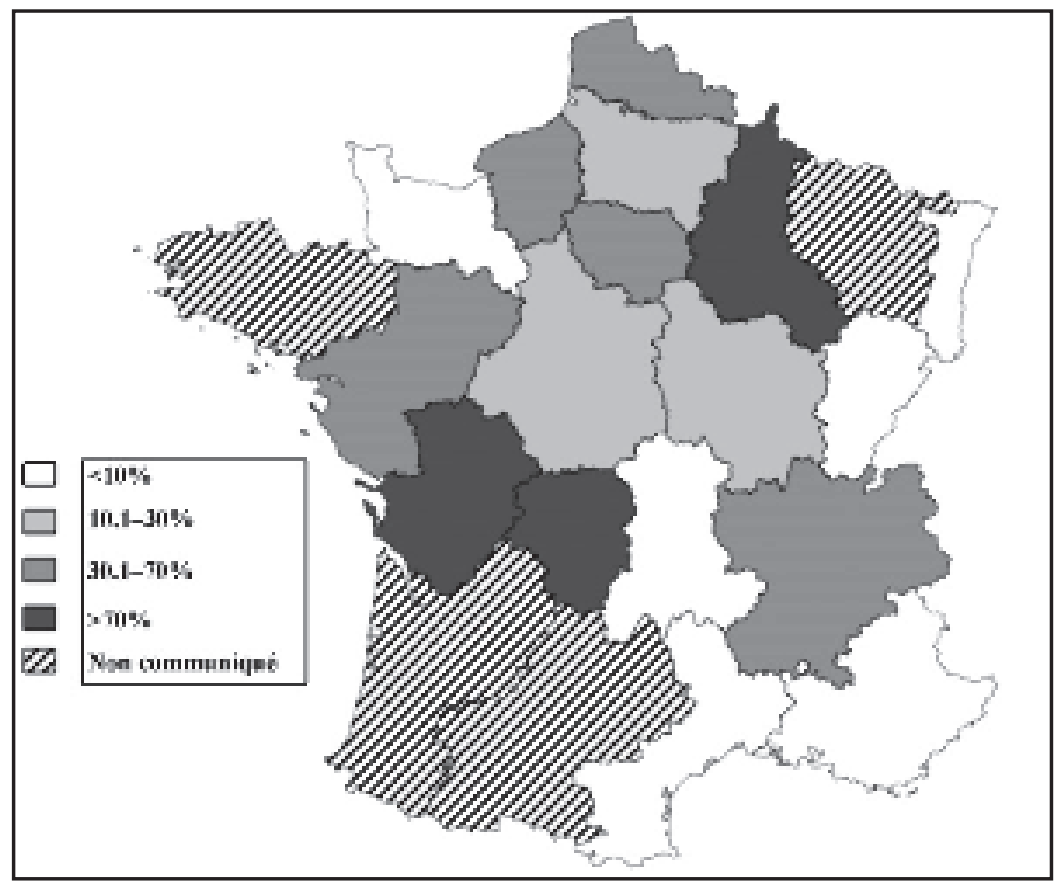

Figure 17 : Distribution géographique régionale de la part des forceps dans l'extraction instrumentale en CHU de niveau III en 2007.

Le forceps n'est pas l'instrument préférentiel à l'échelle nationale [Figure 17] [84]. 
4) Distribution géographique régionale de la part des ventouses dans l'extraction instrumentale en CHU de niveau III en 2007

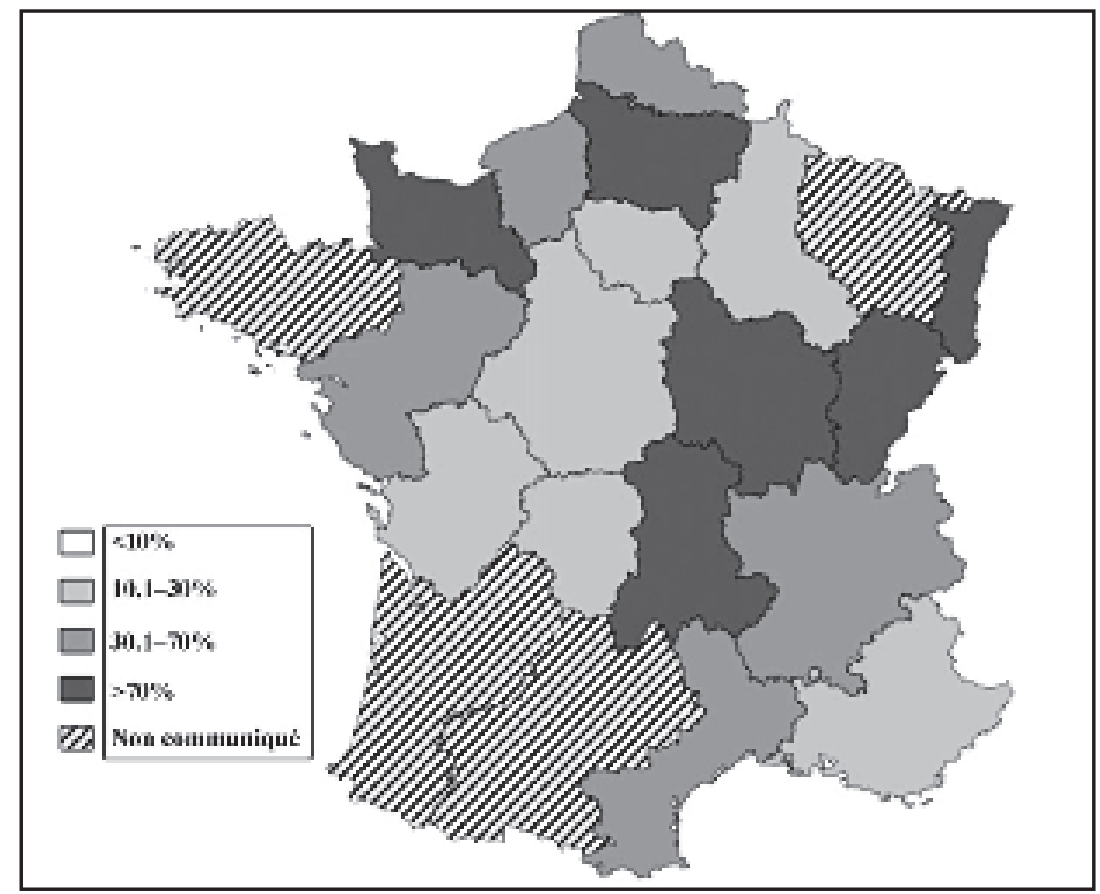

Figure 18 : Distribution géographique régionale de la part des ventouses dans l'extraction instrumentale en CHU de niveau III en 2007.

On observe une nette progression de l'utilisation de la ventouse obstétricale à l'échelle nationale si l'on compare l'état des lieux donné par Schaal et al. en 1993 [86] à celui rapporté en 2007 [figure 18] [84]. Cette modification de la répartition s'est effectuée en une quinzaine d'année au détriment des forceps et de nombreux CHU qui l'utilisaient historiquement de façon quasi exclusive ont largement recours actuellement à la ventouse obstétricale. Le contexte médico légale a probablement influencé cette évolution.

La tendance à la diffusion large de la ventouse s'inscrit dans un mouvement mondial : il a été décrit aux Etats-Unis depuis les années 90 [76,87], ainsi qu'en Europe du Nord [88,89]. 


\title{
H - QUEL INSTRUMENT FAUT-IL APPRENDRE?
}

\author{
1) Que recommande le Collège National des Gynécologues et Obstétriciens \\ français?
}

Les RPC du CNGOF concernant la formation des EI ont été rappelées en 2008 [16].

$\mathrm{Au}$ vu des avantages et limites de chaque instrument, leur utilisation est soumise à un cahier des charges et à un apprentissage spécifique.

○ Le choix de l'instrument est fonction de la situation obstétricale et des préférences de l'obstétricien (accord professionnel).

- Ils doivent permettre à l'obstétricien(ne) de poser ou de réfuter une indication, de choisir l'instrument, de l'utiliser correctement et de connaître les principes du contrôle qualité appliqués à l'extraction instrumentale.

- La formation doit comprendre à la fois l'apprentissage du forceps, de la ventouse obstétricale, et si possible des spatules (complémentarité des instruments). Elle repose sur une parfaite connaissance de la mécanique obstétricale.

- La formation traditionnelle peut aujourd'hui être complétée par un apprentissage par simulation. La formation par simulation pallie aussi au fait que le nombre souhaitable de procédures réalisées est difficilement atteint. La formation devrait être individualisée et prolongée pour certains étudiants.

- La dangerosité de l'extraction instrumentale réside plus dans la qualification de l'opérateur que dans l'instrument utilisé.

○ L'évaluation de la formation concerne à la fois les apprenants et les formateurs.

- Des séances d'évaluation des pratiques professionnelles, au niveau des services, devraient être réalisées et comprendre des critères qualité liés aux extractions. Une telle démarche par le seul fait qu'elle implique les obstétriciens peut améliorer les pratiques (effet Hawthorne). 


\section{2) A l'échelle mondiale : que recommande-t-on ?}

Les éléments déterminants concernant le choix de l'instrument pour une extraction et qui émanent des recommandations anglo-saxonnes (qui comparent le forceps et la ventouse) : sont la variété et la hauteur de la présentation fœtale, ainsi que l'expérience de chaque opérateur [26,87].

Dès 2000, le RCOG recommandait que « seuls les médecins qui ont été correctement formés ou sous supervision d'un médecin correctement formé puissent réaliser une EI » [90].

La méta-analyse de Johanson et al. suggère une complémentarité des instruments : moindre nocivité périnéale de la ventouse et meilleure efficacité du forceps (NP2) [91].

Une enquête réalisée chez plus de 500 internes d'obstétrique a montré que plus de $86 \%$ d'entre eux souhaitent apprendre l'usage du forceps et plus de $97 \%$ l'usage de la ventouse (NP4) [92].

En 2000, O. Driscoll concluait que comme n'importe lequel des instruments d'extraction, le taux de succès de la ventouse était proportionnel à l'expérience de l'opérateur [93,94]. En effet les échecs de ventouse sont dans $40 \%$ des cas liés à une pose incorrecte (NP4) [90].

Il paraît actuellement indispensable de continuer à former les jeunes obstétriciens à la pratique de plusieurs instruments afin de pouvoir choisir en condition pratique le plus adapté à la situation [91]. La complémentarité des instruments d'EI explique pourquoi il est nécessaire d'assurer une formation comportant à la fois l'apprentissage du forceps, de la ventouse et de spatules.

La formation de tout obstétricien doit comprendre l'apprentissage du forceps, de la ventouse et des spatules. 


\section{III - MATERIELS ET METHODES}

\section{A - POPUlation À L'ÉTUDE}

Cette étude prospective mono centrique a été menée dans le service de gynécologie obstétrique, reproduction et médecine fœtale du CHU Nice (maternité de niveau III). Dans ce service les spatules de Thierry sont l'instrument de prédilection pour réaliser les extractions instrumentales.

\section{$>$ Les critères d'inclusion des extractions}

Etaient l'utilisation des spatules de Thierry pour la réalisation d'un accouchement vaginal assisté d'un fœetus singleton, en présentation céphalique, quel que soit le terme, quel que soit le niveau de la présentation dans l'excavation pelvienne par l'un des internes volontaires pour participé à l'étude.

\section{Les critères d'exclusions}

Etaient l'absence de remplissage immédiat de la grille d'évaluation par l'obstétricien de garde (ODG).

Les extractions par spatules dans un contexte de césarienne, de grossesse gémellaire ou de mort fœtale in utero n'étaient pas évaluées.

\section{Condition de réalisation d'une EI}

La décision de réaliser une EI était exclusivement prise par l'ODG, sur la base des RPC du CNGOF [16], à savoir tête fœtale engagée avec au moins une des conditions suivantes :

- suspicion d'hypoxie ;

- non progression de la présentation malgré les efforts expulsifs maternels (durée limitée à 30 minutes) ;

- contre-indication maternelle aux efforts expulsifs

L'ODG devait être présent à chaque extraction. Il validait l'indication, le niveau d'engagement et la variété de la présentation. 


\section{B - PREMIÈRE PHASE DE L'ÉTUDE}

Entre mai 2010 et novembre 2010, une première phase de validation d'un questionnaire d'évaluation des extractions par spatules de Thierry par les internes, tout semestre confondu, était mise en place.

Tous les internes en stage dans le service pendant cette période étaient volontaires pour participer à l'étude. L'ancienneté de l'interne (nombre de semestres validés) était prise en compte. En se basant sur des travaux antérieurs [12], l'interne était considéré comme «junior»s'il avait validé moins de 5 semestres et comme «senior »s'il avait validé 5 semestres ou plus.

\section{C - DEUXIÈME PHASE DE L'ETUDE}

Dans un deuxième temps, entre novembre 2010 et mai 2012, la progression de 6 internes en début de cursus, qui avaient validé moins de 4 semestres au moment de leur inclusion dans l'étude, a été suivie.

Cette étude a suivi les recommandations de la déclaration d'Helsinki 2000 de l'Association médicale mondiale. Chaque interne volontaire pour participer à l'étude signait un formulaire de consentement. Les données étaient traitées de manière dénominalisée.

\section{D - GRILLES D'ÉVALUATION}

Pour les 2 phases de l'étude, chaque procédure auxquelles les internes avaient participé devait être évaluée par l'ODG à l'aide d'une grille systématique complétée immédiatement après un accouchement. [Annexe 1] En parallèle, l'interne remplissait un court questionnaire sur sa propre perception de ses capacités au moment de la procédure. [Annexe 2]

La grille d'évaluation a été construite sur le modèle d'un audit clinique sur la base des objectifs de la formation aux EI décrits par Dupuis [85] et Sentiles [24] et de la grille d'évaluation des accouchements instrumentaux du RCOG [26] [Annexe 3]. La validité de contenu de cette grille a été vérifiée grâce à l'avis de 3 experts (plus de 10 ans d'expérience 
des accouchements assistés par spatules) qui ont confirmé la représentativité des différents items de la grille.

Cette fiche est simple, rapide à compléter et exhaustive, permettant d'attribuer une note de 1 à 5 selon la qualité de l'information donnée à la patiente avant le geste, de la préparation avant le geste, de chacune des étapes de l'extraction, du dégagement du mobile fœetal et du contrôle du périnée. Un score total est calculé en faisant la somme des points obtenus à chaque item. Un item final permet de donner une appréciation du niveau de compétence globale de l'interne pour la réalisation de l'extraction [Annexe1].

Indépendamment, en utilisant un questionnaire inspiré du travail de Jude et al. [104], les internes auto-évaluaient la qualité de leur extraction et leur sentiment de confiance quant à leur capacité à réaliser cette procédure de manière autonome [Annexe 2].

\section{C - RECUEIL DES DONNÉES}

En accord avec le protocole du service, immédiatement après l'accouchement, un compte rendu type était complété par l'interne et/ou l'ODG et signé par ce dernier pour chaque extraction par ST [Annexe 4].

Les informations suivantes y figuraient systématiquement : nom du médecin responsable et de l'interne, gestité, parité, âge gestationnel, indication de l'extraction instrumentale, durée du travail et des efforts expulsifs, type d'anesthésie, modalités d'engagement et de dégagement de la présentation, lésions périnéales, vaginales, cervicales ou péri urétrales et épisiotomie. Le sexe et l'Apgar du nouveau né devaient être également mentionnés.

Les déchirures périnéales étaient identifiées de manière distincte des épisiotomies selon les critères de la Classification internationale des maladies de l'OMS [45].

Les comptes-rendus de toutes les extractions par spatules de Thierry réalisées par les internes volontaires pour participer à l'étude ont été collectés [Annexe 4]. 


\section{D - ANALYSE STATISTIQUE}

Les données étaient centralisées et anonymisées dans une base de donnée informatique. Tous les tests statistiques ont été réalisés sous l'hypothèse bilatérale au seuil de signification de 0.05 avec le logiciel SPSS 19.0.0 (SPSS Inc. C), Chicago, IL 1989-2010).

\section{lère phase de l'étude}

Une analyse descriptive des caractéristiques de l'échantillon a d'abord été réalisée, ainsi qu'une comparaison entre les caractéristiques des extractions par spatules de Thierry évaluées et celles des extractions non évaluées (test exact de Fisher ou test de Mann Whitney).

Différentes analyses univariées ont ensuite été réalisées pour tester l'association entre ancienneté de l'interne (internes «seniors » vs « juniors»), difficulté de l'extraction, niveau de confiance, besoin d'aide verbale ou manuelle, sensation de progression, score total, niveau de compétence globale, et taux de taux de lésions périnéales sévères en utilisant les test de Chi2 de Pearson et de Fisher pour les variables catégorielles et le test non paramétrique de Mann-Whitney pour les variables continues.

Enfin les scores totaux et les niveaux de compétence globale des évaluations réalisés par les 2 ODG qui en avaient réalisé le plus ont été comparés.

\section{2ème phase de l'étude}

Après un traitement initial des données initial à ce celui de la premier étape, l'impact de l'ancienneté des internes et de leur niveau d'expérience (catégorisé en fonction du nombre d'extraction par spatules réalisé avant l'accouchement évalué : plus de 20 versus 20 et moins) sur le score total selon l'ODG ont été explorés par les mêmes tests univariés. Un modèle linéaire mixte (MLM) à effet aléatoire a permis d'étudier de manière multivariée l'impact de l'ancienneté de l'interne sur le score total et l'évaluation globale de l'interne, en considérant l'identité de l'interne comme un facteur ayant un effet aléatoire, et en prenant en compte la difficulté de l'extraction. De la même façon, il a été étudié l'impact sur le score total du fait d'avoir ou non réalisé au moins 20 extractions par spatules avant l'extraction évaluée. 


\section{IV - RESULTATS DE NOTRE ETUDE}

\section{A - RÉSULTATS DE LA 1ÈRE PHASE DE L'ETUDE}

Entre mai et novembre 2010, nous avons comptabilisé 1499 accouchements dans notre service. Les spatules étaient l'instrument utilisé dans 98\% (n=108) des accouchements vaginaux assistés [Figure 19]. La grille d'évaluation a été remplie par l'ODG dans 27 cas soit 26,2\% des extractions par spatules, tandis que l'auto-questionnaire des internes a été complété dans 28 cas $(27,1 \%)$.

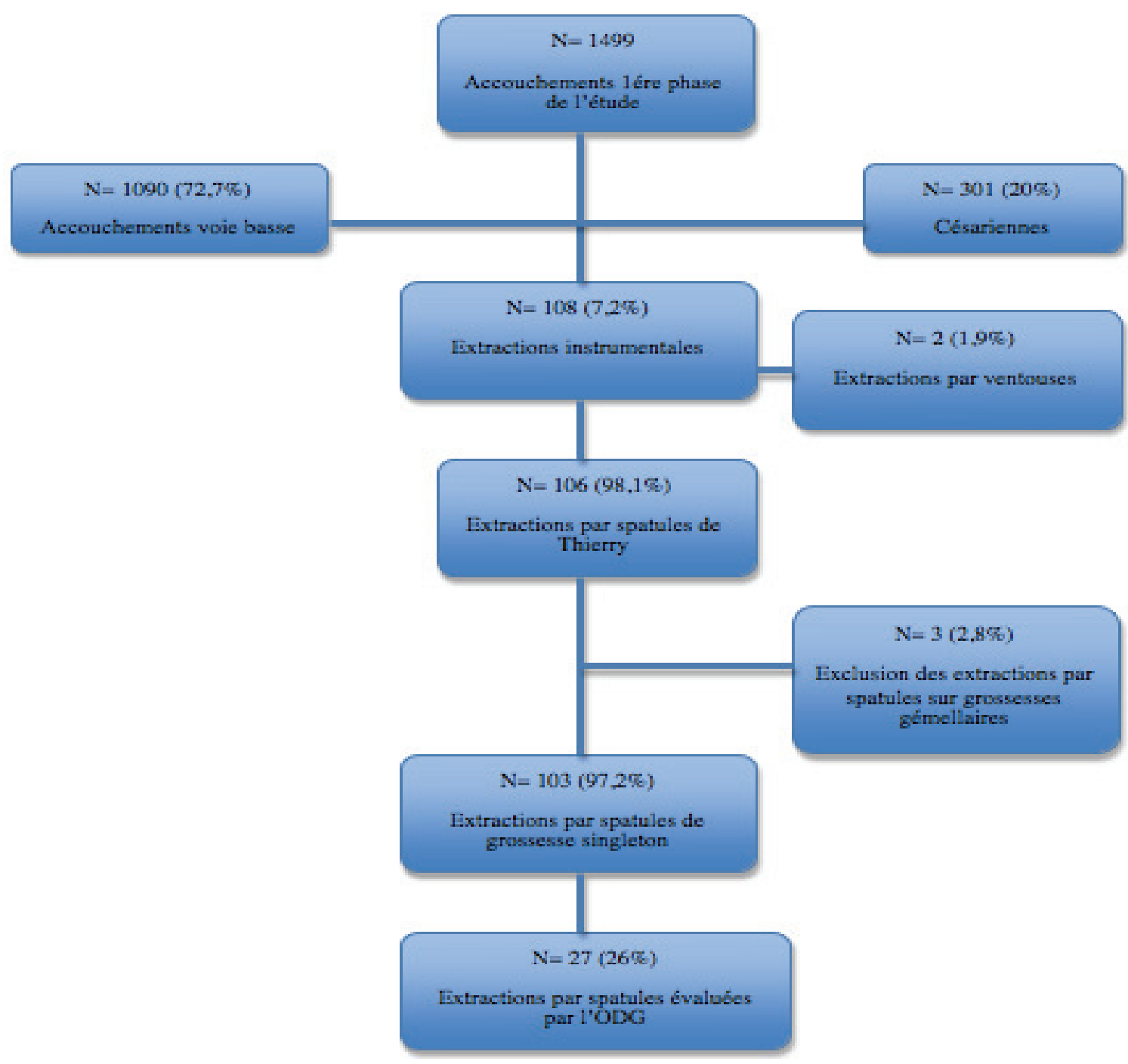

Figure 19 : Diagramme de sélection des extractions par spatules évaluées dans la première partie de l'étude 


\section{1) Description : caractéristiques maternelles, obstétricales et néonatales}

Les caractéristiques maternelles, obstétricales et néonatales des sujets ayant fait l'objet d'une extraction par spatules sont présentées dans le tableau 7.

Les caractéristiques des femmes dont l'accouchement assisté a été évalué ne sont pas significativement différentes des caractéristiques de l'ensemble des femmes ayant eu un accouchement vaginal assisté par spatules sur la période d'étude. Concernant la prise en charge obstétricale, la seule différence significative entre les extractions évaluées et les autres concerne le taux de révision utérine, qui est significativement inférieur parmi les cas évalués $(23,4$ vs $7,4 \%, p=0,032)$.

Le taux de lésions néonatales était de 12,4\% dans le groupe évalué versus 8,7\% dans le groupe non évalué, sans que cette différence ne soit significative. Les 2 cas de lésions néonatales attribuables aux spatules étaient des atteintes superficielles cutanées d'évolution rapidement favorable de type érythème ou excoriation ; ces atteintes n'ont dans aucun des cas été responsables d'une prolongation de l'hospitalisation du nouveau né ou de conséquences à long terme. 
Tableau 7 : Caractéristiques, maternelles, obstétricales et néonatales de la première phase de l'étude (mai à novembre 2010).

\begin{tabular}{|c|c|c|c|}
\hline Variables* & $\begin{array}{l}\text { Toutes les extractions } \\
n=103\end{array}$ & $\begin{array}{c}\text { Extractions } \\
\text { évaluées } \\
n=27\end{array}$ & $\mathbf{p}^{* *}$ \\
\hline Âge (année) & $29(25-32)$ & $28(24-31)$ & 0,437 \\
\hline Nullipare & $67(69,8)$ & $21(77,8)$ & 0,332 \\
\hline Age gestationnel (semaine d'aménorrhée) & $40,4(39,2-41,0)$ & $40,5(39,4-41,3)$ & 0,183 \\
\hline Déclenchement & $18(19,4)$ & $5(18,5)$ & 1,000 \\
\hline Durée du travail (h) & $7(5,0-8,6)$ & $6,3(5,0-8,2)$ & 0,810 \\
\hline Durée des efforts expulsifs (min) & $20(20-25)$ & $20(18-26)$ & 0,690 \\
\hline Anesthésie péridurale ou rachianesthésie & $87(87,9)$ & $25(92,6)$ & 0,175 \\
\hline Indication de l'extraction & & & 1,000 \\
\hline EEI & $66(64,7)$ & $18(66,7)$ & \\
\hline ARCF & $9(8,8)$ & $2(7,4)$ & \\
\hline EEI et ARCF & $25(24,5)$ & $7(25,9)$ & \\
\hline Protection fotale & $1(1)$ & $0(0)$ & \\
\hline Contre indication maternelle & $1(1)$ & $0(0)$ & \\
\hline Extraction au détroit moyen & $94(91,3)$ & $26(96,3)$ & 0,439 \\
\hline Présentations transverses ou postérieures & $12(11,7)$ & $5(18,5)$ & 0,302 \\
\hline Dégagement en occipito-sacré & $18(17,5)$ & $2(7,4)$ & 0,143 \\
\hline Retrait des spatules au petit couronnement & $29(90,6)$ & $22(88,0)$ & 1,000 \\
\hline Césarienne & $1(1,0)$ & $0(0)$ & 1,000 \\
\hline Révision utérine & $24(23,4)$ & $2(7,4)$ & 0,032 \\
\hline Episiotomie & $68(66)$ & $18(66,7)$ & 1,000 \\
\hline Lésion périnéale sévère & $10(9,7)$ & $3(11,1)$ & 0,719 \\
\hline Déchirure vaginale & $42(40,8)$ & $12(44,4)$ & 0,656 \\
\hline Déchirure péri-urétrale & $1(1,0)$ & $1(3,7)$ & 0,262 \\
\hline Déchirure cervicale & $2(1,9)$ & $0(0)$ & 1,000 \\
\hline Poids des nouveau-nés (gramme) & $3460(3160-3760)$ & $3440(3140-3710)$ & 0,961 \\
\hline Apgar <3 à 1 minute & $3(2,9)$ & $1(3,7)$ & 1,000 \\
\hline Apgar <7 à 5 minutes & $3(2,9)$ & $1(3,7)$ & 1,000 \\
\hline Lésions néonatales & $2(8,7)$ & $2(12,4)$ & 1,000 \\
\hline
\end{tabular}


* Les médianes (25e-75e percentiles) sont reportées pour les variables continues et les nombres absolus $(\%)$ pour les variables catégorielles.

** Test exact de Fisher ou test de Mann Whitney.

ARCF : anomalie du rythme cardiaque fotal EEI : efforts expulsifs inefficaces

\section{2) Profil des internes évalués}

En moyenne les 11 internes avaient validé 5 semestres dont 3 en gynécologie obstétrique. Les plus jeunes internes avaient validé un seul semestre de l'internat; ce semestre s'était déroulé dans un service de gynécologie obstétrique. Les plus vieux internes en avaient validé 9 au total dont 7 dans un service de gynécologie obstétrique. Le sexe ratio des internes était d'un homme pour 10 femmes.

Les internes ont participé à un nombre moyen d'extraction par spatules de 9,1 +/-1,5 sur les 6 mois d'étude. La distribution du nombre d'extractions évaluées en fonction de l'ancienneté des internes qui les ont réalisées est présentée dans la figure 20 : 12 évaluations ont concerné des internes juniors (moins de 5 semestres validés), 15 des internes seniors (5 semestres et plus validés).

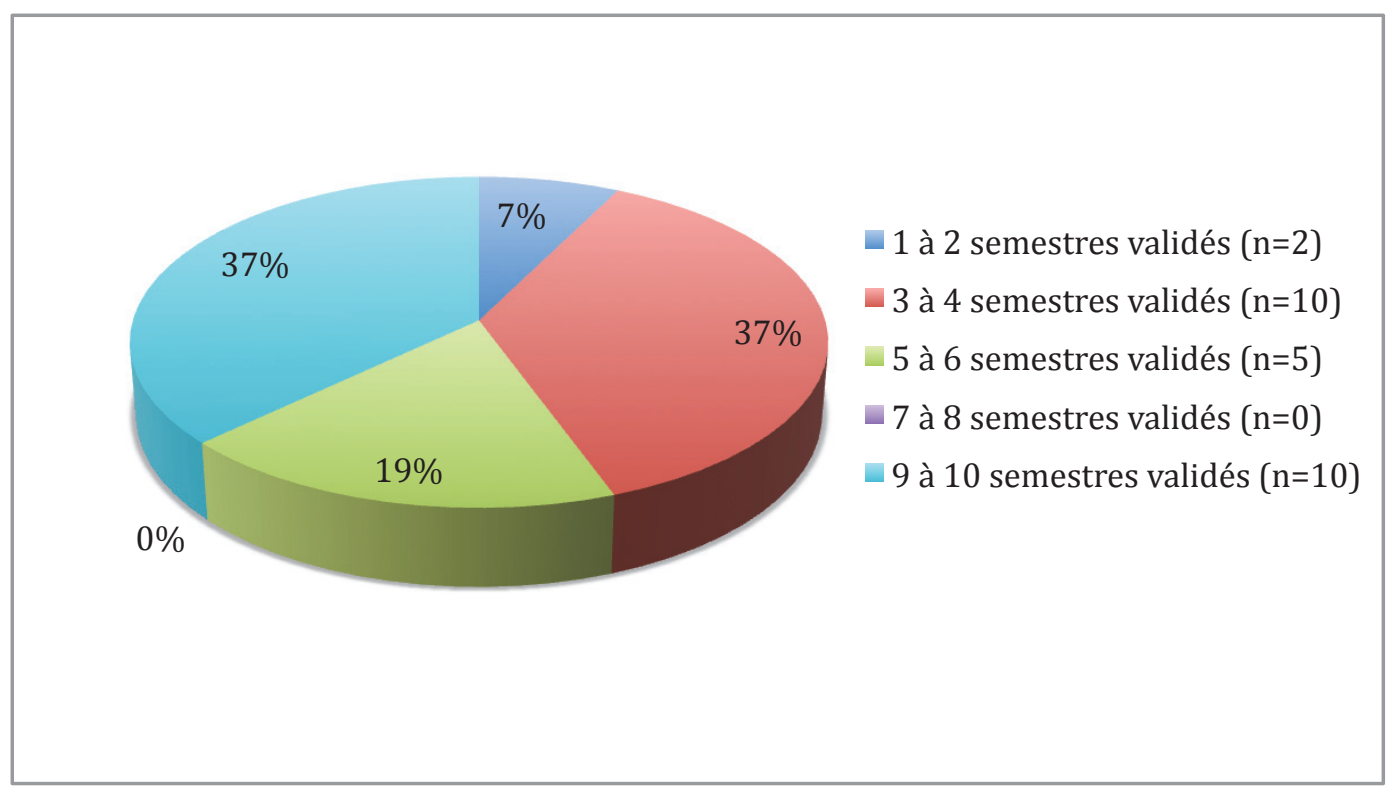

Figure 20 : Nombre d'extractions évaluées en fonction de l'ancienneté des internes. 


\section{3) Auto-questionnaire des internes}

Lors des extractions réalisées par les internes, l'ODG était présent dans 94,4\% des cas.

Selon les internes, une aide verbale était apportée dans 55,6\% des cas et une aide manuelle dans $15,4 \%$ des cas. L'aide verbale était significativement plus fréquente chez les internes «junior» comparé aux internes «senior» (80\% versus $41 \% \mathrm{p}=0,05)$. Une aide manuelle était aussi plus fréquemment nécessaire quand l' interne était « junior », sans que la différence soit significative $(37 \%$ versus $6 \%, \mathrm{p}=0,072)$. Cette aide manuelle ne concernait jamais plus de 2 étapes de l'accouchement; il s'agissait le plus souvent de la traction/propulsion (36\%), du retrait de l'instrument (31\%), et de l'épisiotomie (21\%).

$>$ Les internes ont étiqueté $27,6 \%$ des extractions comme difficile ou très difficile.

La sensation de progression dans l'acquisition du geste d'extraction par spatules était ressenti par les internes lors de 72,4\% des extractions évaluées.

Lors de 46,4\% des accouchements, les internes évalués ne se sentaient pas apte à la réalisation d'une extraction sans supervision. Quinze procédures ont été réalisées par un interne qui se sentait apte à réaliser l'extraction de manière indèpendante, dont 4 cas où l'interne considérait qu'il aurait eu besoin de soutien en cas de complication. Les internes «senior» étaient plus en confiance pour réaliser de maniére indépendante l'extraction par spatules, comparés au groupe des internes « juniors » $(77,8 \%$ versus $10,0 \% ; \mathrm{p}=0,001)$.

\section{4) Évaluations par les obstétriciens de garde}

La moyenne des scores obtenus aux différents items de la grille d'évaluation était supérieure ou égale à 4 pour chacun des items ; l'axe de traction et la réalisation de l'épisiotomie étaient les étapes de l'accouchement qui semblaient être les plus challengantes pour les internes. Le score total moyen était de 4,2+/-0,8 sur 5 [Tableau 8] .

Les internes «seniors » avaient tendance à avoir un meilleur score total que les «juniors » (mediane 4,7 versus 4,3; $\mathrm{p}=0,068$ ). Lorsque l'extraction était considérée comme difficile par l'interne, leurs scores totaux avaient tendance à être inférieurs (médiane 4,1 vs 4,8; p=0, 079). 
Tableau 8 : Résultats à chaque item de la grille d'évaluation des internes par les obstétriciens de garde.

\begin{tabular}{|l|c|}
\hline & Moyenne, Écart type, étendu \\
\hline Analgésie & $4,4+/-0,9(2-5)$ \\
\hline Dilatation complète & $4,8+/-0,6(2-5)$ \\
\hline Asepsie & $4,5+/-0,8(2-5)$ \\
\hline Evaluation du niveau d'engagement & $4,5+/-1(1-5)$ \\
\hline Evaluation de la présentation & $4,5+/-1,6(1-5)$ \\
\hline Sondage urinaire évacuateur & $4,6+/-0,7(2-5)$ \\
\hline Information de la patiente & $4,2+/-1,1(1-5)$ \\
\hline Mouvement de pose lére spatule & $4,2+/-1,1(1-5)$ \\
\hline Mouvement de pose de la 2ème spatule & $4,1+/-1,1(1-5)$ \\
\hline Protection des tissus mous à la pose & $4,3+/-1,1(1-5)$ \\
\hline Extraction coordonnée avec EE & $4,3+/-0,9(2-5)$ \\
\hline Axe de traction correct & $4+/-0,9(2-5)$ \\
\hline Propulsion & $4,1+/-0,9(2-5)$ \\
\hline Asynclitisme & $4,2+/-0,8(2-5)$ \\
\hline Geste de dégagement de la tête & $4,5+/-0,8(2-5)$ \\
\hline Contrôle du périnée postérieur & $4+/-1(2-5)$ \\
\hline Geste de dégagement du corps & $4,4+/-0,9(2-5)$ \\
\hline Retrait des spatules & \\
\hline Episiotomie & $4+/-0,9(2-5)$ \\
\hline Délivrance & \\
\hline
\end{tabular}


La figure 21 représente la distribution de l'appréciation du niveau de compétence globale de l'interne selon l'ODG. Les internes jugés capables de réaliser une extraction sans supervision avaient des scores globaux supérieurs aux autres (médiane 4,9 versus $4,2 ; \mathrm{p}<0,001)$. Il existait une bonne concordance entre le niveau de compétence globale évalué par l'ODG et le niveau de confiance rapporté par l'interne à chaque extraction (Kappa=0,8; $\mathrm{p}<0,001)$. Après 64\% des extractions réalisées par des internes « seniors » l'ODG considérait l'interne capable de réaliser une extraction sans supervision, alors qu'il ne portait cette appréciation que dans $9 \%$ des cas lorsque l'interne était « junior» $(p=0,005)$.

Il n'y avait pas de différence significative lorsqu'on comparait les scores totaux des évaluations faites par les 2 ODG qui en avaient réalisé le plus (respectivement 10 et 9), que les internes aient été « senior» (moyenne 4,5 vs 4,7 p=0,167) ou « junior» (moyennes 4,4 et $4,3 ; p=0,329)$. De même il n'y avait pas de différence significative dans leurs évaluations de la compétence globale, que les internes aient été « senior » $(\mathrm{p}=0,491)$ ou « junior » $(\mathrm{p}=0,286)$.

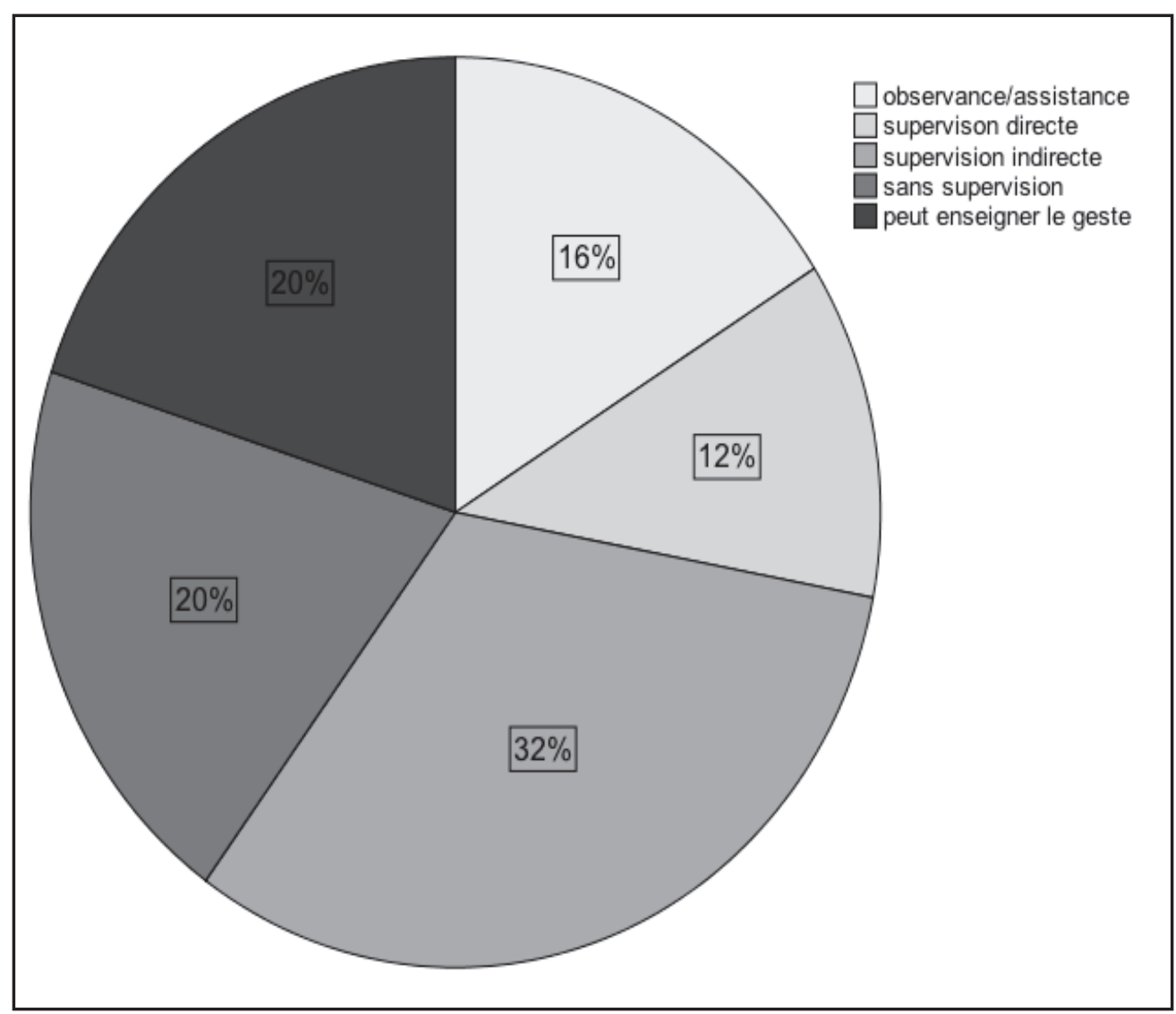

Figure 21 : Évaluation par l'obstétricien de garde du niveau de compétence globale de l'interne pour la réalisation des accouchements vaginaux assistés par spatules de Thierry. 


\section{5) Lien entre ancienneté de l'interne, évaluations et complications maternelles et néonatales}

Le taux de lésion périnéale sévère en fonction du nombre de semestres validés en gynécologie obstétrique est présenté dans la figure 22 : il existe une tendance à la diminution de la fréquence des lésions périnéales sévères avec le gain en ancienneté de l'interne [Figure 22].

Le taux de lésions périnéales sévère était de $13,2 \%$ lorsque l'interne « juniors» et de 6,4\% lorsqu'il était « senior » $(\mathrm{p}=0,328)$.

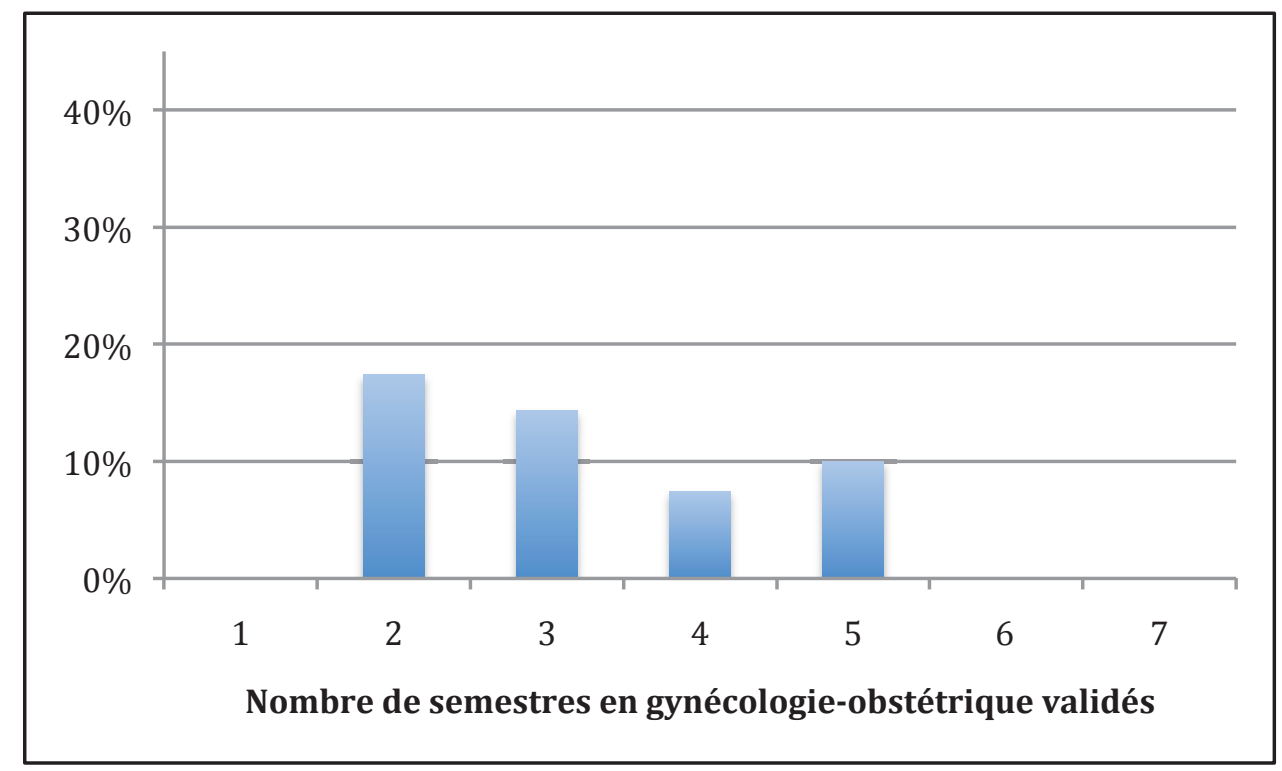

Figure 22 : Taux de lésion périnéale sévère en fonction du nombre de semestres validés en gynécologie obstétrique.

Le score total des internes ne différait pas significativement que les accouchements aient été compliqués de lésion périnéale sévère ou non (médianes 4,5 versus 4,$7 ; p=0,762$ ); cependant le score de l'item concernant spécifiquement la protection du périnée tendait à être inférieur en cas de lésion périnéale sévère $(3,5$ versus 5,$0 ; p=0,08)$. De même le score total de l'interne ne diffère pas significativement en cas de révision utérine (médianes 2,0 versus 4,7; $\mathrm{p}=0,154)$ mais le score concernant spécifiquement la délivrance tend à être inférieur $(1,0$ versus 5,$0 ; \mathrm{p}=0,08)$. 
Le nombre de complications néonatales était insuffisant pour pouvoir étudier leur association avec le score de l'interne.

Le niveau de compétence globale et le niveau de confiance ne différaient pas en fonction des complications maternelles et néonatales [Tableaux 9].

Cependant, le taux de lésions périnéales sévères tendait à être inférieur lorsque l'interne se sentait en confiance pour réaliser des extractions par spatules de manière indépendante $(6,7 \%$ versus $15,4 \% ; \mathrm{p}=0,583)$ et lorsque il ne considérait pas l'extraction comme difficile $(4,8 \%$ versus $25 \% \mathrm{p}=0,176)$. [Tableau 9]

Le taux de déchirures vaginales était significativement supérieur lorsque l'interne considérait l'extraction comme difficile $(75 \%$ versus $28,6 \% \mathrm{p}=0,038)$ sans être associé à un taux d'épisiotomie supérieur comparé aux extractions considérées «faciles » $50 \%$ versus $66,7 \% \mathrm{p}=0,433)$. Les extractions réalisées par les internes «juniors » étaient plus souvent associées à une révision utérine comparées à celle des internes «seniors », sans être significativement associées à d'autres complications maternelles ou néonatales [Tableau 9]. 
Tableau 9 : Comparaison des taux de complications maternelles et néonatales des accouchements assistés par spatules de Thierry en fonction de l'ancienneté de l'interne, de son niveau de compétence globale évalué par l'obstétricien de garde, de son niveau de confiance au moment de l'extraction et la difficulté de la procédure.

\begin{tabular}{|c|c|c|c|c|c|c|}
\hline & $\mathbf{n}$ & $\begin{array}{l}\text { Lésions } \\
\text { périnéales } \\
\text { sévères }\end{array}$ & $\begin{array}{l}\text { Déchirures } \\
\text { vaginales }\end{array}$ & Episiotomie & $\begin{array}{l}\text { Révision } \\
\text { utérine }\end{array}$ & $\begin{array}{l}\text { Complicatio } \\
\text { ns } \\
\text { maternelles } \\
\text { sévères* }\end{array}$ \\
\hline $\begin{array}{l}\geq 5 \text { semestres } \\
<5 \text { semestres } \\
p^{* *}\end{array}$ & $\begin{array}{l}47 \\
53\end{array}$ & $\begin{array}{l}3(6,4) \\
7(13,2) \\
0,328\end{array}$ & $\begin{array}{l}18(38,3) \\
21(39,6) \\
1,000\end{array}$ & $\begin{array}{l}29(61,7) \\
37(69,8) \\
0,407\end{array}$ & $\begin{array}{l}5(10,6) \\
18(34,0) \\
0,008\end{array}$ & $\begin{array}{l}6(12,8) \\
7(13,2) \\
1,000\end{array}$ \\
\hline $\begin{array}{l}\text { Compétent } \\
\text { supervision }\end{array}$ & 10 & $1(10,0)$ & $3(30,0)$ & $7(70,0)$ & $0(0)$ & $2(20,0)$ \\
\hline $\begin{array}{l}\text { Non compétent } \\
\text { sans supervision }\end{array}$ & 15 & $2(13,3)$ & $8(53,3)$ & $10(66,7)$ & $1(6,7)$ & $2(13,3)$ \\
\hline$p^{* *}$ & & 1,000 & 0,414 & 1,00 & 1,000 & 1,000 \\
\hline En confiance & 15 & $1(6,7)$ & $5(33,3)$ & $9(60,0)$ & $2(13,3)$ & $2(13,3)$ \\
\hline $\begin{array}{l}\text { Pas en confiance } \\
p^{* *}\end{array}$ & 13 & $\begin{array}{l}2(15,4) \\
0,583\end{array}$ & $\begin{array}{l}7(53,8) \\
0,445\end{array}$ & $\begin{array}{l}8(61,5) \\
1,000\end{array}$ & $\begin{array}{l}1(7,7) \\
1,000\end{array}$ & $\begin{array}{l}2(15,4) \\
1,000\end{array}$ \\
\hline $\begin{array}{l}\text { Extraction } \\
\text { difficile }\end{array}$ & 8 & $2(25,0)$ & $6(75,0)$ & $4(50,0)$ & $2(25,0)$ & $2(25,0)$ \\
\hline $\begin{array}{l}\text { Extraction non } \\
\text { difficile }\end{array}$ & 21 & $1(4,8)$ & $6(28,6)$ & $14(66,7)$ & $1(4,8)$ & $2(9,5)$ \\
\hline$p^{* *}$ & & 0,176 & 0,038 & 0,433 & 0,176 & 0,300 \\
\hline
\end{tabular}

En confiance : en confiance pour réaliser des accouchements vaginaux assistés par spatules de Thierry de manière indépendante.

AVA : accouchement vaginal assisté par spatules

*Les complications maternelles sévères incluent: lésions périnéales du $3^{\text {ème }}$ degré et plus $(\mathrm{n}=10)$, hémorragies de la délivrance $(\mathrm{n}=3)$, thrombus vaginal $(\mathrm{n}=1)$

** test exact de Fisher 


\section{B - RÉSULTATS DE LA $2^{\text {Ème }}$ PHASE}

Cette phase de l'étude consistait au suivi de l'apprentissage des spatules par 6 internes en GO, entre novembre 2010 et mai 2011.

Toutes ces internes étaient des femmes ; 2 ont été suivies sur une période d'un an et demi, 3 sur une période d'un an et 1 sur une période de 6 mois. Au début du suivi, 4 des 6 internes étaient en premier semestre et les deux autres avaient déjà validé deux semestres, les deux en GO.

Sur la période d'étude, ces 6 internes ont participé à 160 accouchements vaginaux assistés par spatule, dont $63(39,6 \%)$ ont été évalués. En moyenne chaque interne a réalisé 12+/-6 extractions par semestre où il était présent dans le service de GO du CHU et a été évalué 4,8 fois par semestre.

\section{1) Description : caractéristiques maternelles, obstétricales et néonatales}

Les caractéristiques des mères et des nouveaux-nés n'étaient pas significativement différentes, qu'il y ait eu ou non une évaluation au moment de l'accouchement [Tableau 10].

Les 5 lésions néonatales décrites attribuables à l'extraction par spatules étaient des ecchymoses temporales ou des excoriations cutanées superficielles d'évolution rapidement favorable. 
Tableau 10 : Caractéristiques, maternelles, obstétricales et néonatales de la seconde phase de l'étude (novembre 2010 à mai 2012).

\begin{tabular}{|l|c|c|c|}
\hline Variables* & $\begin{array}{c}\text { Toutes les } \\
\text { extractions } \\
\mathbf{n = 1 6 0}\end{array}$ & $\begin{array}{c}\text { Extractions } \\
\text { évaluées } \\
\text { n=63 }\end{array}$ & p** \\
\hline Âge (année) & $27(24-32)$ & $27(25-32)$ & 0,726 \\
\hline Nullipare & $119(74,4)$ & $42(66,7)$ & 0,095 \\
\hline Age gestationnel (semaine d'aménorrhée) & $40,3(39,1-$ & $40,0(39,0-41,0)$ & 0,297 \\
\hline Déclenchement & $41,0)$ & & \\
\hline Durée du travail (h) & $40(25,0)$ & $15(23,8)$ & 0,853 \\
\hline Durée des efforts expulsifs (min) & $6(4,5-8,5)$ & $6,0(5,0-8,5)$ & 0,340 \\
\hline Anesthésie péridural ou rachianesthésie & $20(15-25)$ & $20(17-25)$ & 0,223 \\
\hline Indication de l'extraction & $155(96,9)$ & $63(100)$ & 0,158 \\
\hline EEI & & & 0,519 \\
\hline ARCF & $86(53,8)$ & $36(57,1)$ & \\
\hline EEI et ARCF & $36(22,5)$ & $13(20,6)$ & \\
\hline Extraction au détroit moyen & $38(23,8)$ & $14(22,2)$ & \\
\hline Présentations transverses ou postérieures & $138(86,3)$ & $55(87,3)$ & 0,818 \\
\hline Dégagement en OS & $14(9,3)$ & $7(11,5)$ & 0,569 \\
\hline Césarienne & $29(18,5)$ & $11(17,7)$ & 1,000 \\
\hline Révision utérine & $4(2,5)$ & $1(1,6)$ & 1,000 \\
\hline Episiotomie & $34(21,2)$ & $12(19,0)$ & 0,693 \\
\hline Lésion périnéale sévère & $103(64,4)$ & $40(63,5)$ & 0,867 \\
\hline Déchirure vaginale & $8(5,0)$ & $3(4,8)$ & 1,000 \\
\hline Déchirure péri urétrale & $75(46,9)$ & $34(54,0)$ & 0,194 \\
\hline Déchirure cervicale & $2(1,3)$ & $1(1,6)$ & 1,000 \\
\hline Poids des nouveau-nés (gramme) & $3(1,9)$ & $1(1,6)$ & 1,000 \\
\hline Apgar <3 à 1 minute & 3415 & 3490 & 0,720 \\
\hline Apgar <7 à 5 minutes & $0(0)-3765)$ & $(3025-3765)$ & \\
\hline Lésions néonatales, n (\%) & $0(0)$ & 1,000 \\
\hline
\end{tabular}

* Les médianes (25e-75e percentiles) sont reportées pour les variables continues et les nombres absolus (\%) pour les variables catégorielles.

** Test exact de Fisher ou test de Mann Whitney.

ARCF : anomalie du rythme cardiaque fetal EEI : efforts expulsifs inefficaces 


\section{2) Auto-questionnaires des internes}

L'ODG était présent dans 94\% des extractions réalisées par les internes.

Selon les internes, il leur avait apporté une aide verbale dans $77 \%$ des cas et une aide manuelle dans $65 \%$ des cas. Cette aide correspondait à plus de la moitié des étapes de l'extraction instrumentale dans $23 \%$ des accouchements.

Les internes ont jugé $21 \%$ des extractions comme difficile ou très difficile.

La sensation de progression dans l'acquisition du geste d'extraction par spatules était ressentie lors de $91 \%$ des accouchements par les internes.

Dans $84 \%$ des accouchements, l'interne se sentait apte à la réalisation d'une extraction avec supervision rapprochée ou minime, tandis que dans $14 \%$ des cas, il ne se sentait pas du tout en confiance.

\section{3) Evaluations par les obstétriciens de garde}

Le score total moyen était de 3,8 +/-0,7.

Les étapes les plus problématiques pour les internes étaient l'information de la patiente, le mouvement de pose de la seconde spatule l'axe de traction la propulsion l'asynclitisme et la réalisation de l'épisiotomie [Tableau 10]. Le coefficient alpha de Cronbach des différents items de la grille d'évaluation était de 0,99.

L'ODG considérait l'interne capable de réaliser des extractions sous supervision directe ou indirecte lors de $69 \%$ et $18 \%$ des accouchements respectivement ; aucun interne n'a été jugé capable de réaliser des extractions sans supervision durant la période de suivi. 
Tableau 11 : Résultats à chaque item de la grille d'évaluation des internes par l'obstétricien de garde (2ème phase d'étude).

\begin{tabular}{|c|c|}
\hline & Moyenne, Ecart-type, étendu \\
\hline Analgésie & $4,3+/-0,8(1-5)$ \\
\hline Dilatation complète & $4,6+/-0,8(1-5)$ \\
\hline Asepsie & $4,5+/-0,8(1-5)$ \\
\hline Evaluation du niveau d'engagement & $4+/-0,9(1-5)$ \\
\hline Evaluation de la présentation & $3,5+/-1(1-5)$ \\
\hline Sondage urinaire évacuateur & $4,8+/-0,4(3-5)$ \\
\hline Information de la patiente & $3,4+/-1(1-5)$ \\
\hline Mouvement de pose 1ére spatule & $3,6+/-1(1-5)$ \\
\hline Mouvement de pose de la 2ème spatule & $3,4+/-1(1-5)$ \\
\hline Protection des tissus mous à la pose & $3,6+/-1(1-5)$ \\
\hline Extraction coordonnée avec EE & $3,5+/-1(2-5)$ \\
\hline Axe de traction correct & $3,3+/-1(1-5)$ \\
\hline Propulsion & $3,3+/-1(1-5)$ \\
\hline Asynclitisme & $3,1+/-0,9(1-5)$ \\
\hline Geste de dégagement de la tête & $3,6+/-0,9(1-5)$ \\
\hline Contrôle du périnée postérieur & $3,5+/-1(1-5)$ \\
\hline Geste de dégagement du corps & $3,7+/-1(2-5)$ \\
\hline Retrait des spatules & $3,5+/-1,1(1-5)$ \\
\hline Episiotomie & $3,2+/-1,1(1-5)$ \\
\hline Délivrance & $4,4+/-0,8(1-5)$ \\
\hline Utérus tonique & $4,3+/-0,8(1-5)$ \\
\hline Evaluation des lésions périnéales & $4,3+/-0,8(1-5)$ \\
\hline Pertes sanguines & $4,1+/-0,9(1-5)$ \\
\hline Interaction avec le personnel & $3,9+/-1,1(1-5)$ \\
\hline
\end{tabular}




\section{C - COURBE D'APPRENTISSAGE}

\section{1) Impact de l'ancienneté de l'interne sur leurs résultats aux évaluations}

La figure 23 représente les scores totaux moyens des internes, en fonction de leur ancienneté (nombre de semestres validés) au moment de chaque accouchement assisté par spatules pour lequel ils ont été évalués. Les moyennes des scores globaux sont 3,5, 3,2, 4,4 et 4,6 sur 5 après respectivement $0,1,2$ et 3 semestres validés ( $p<0,001)$. Il n'y a pas d'impact significatif de la difficulté de l'extraction sur cette relation.

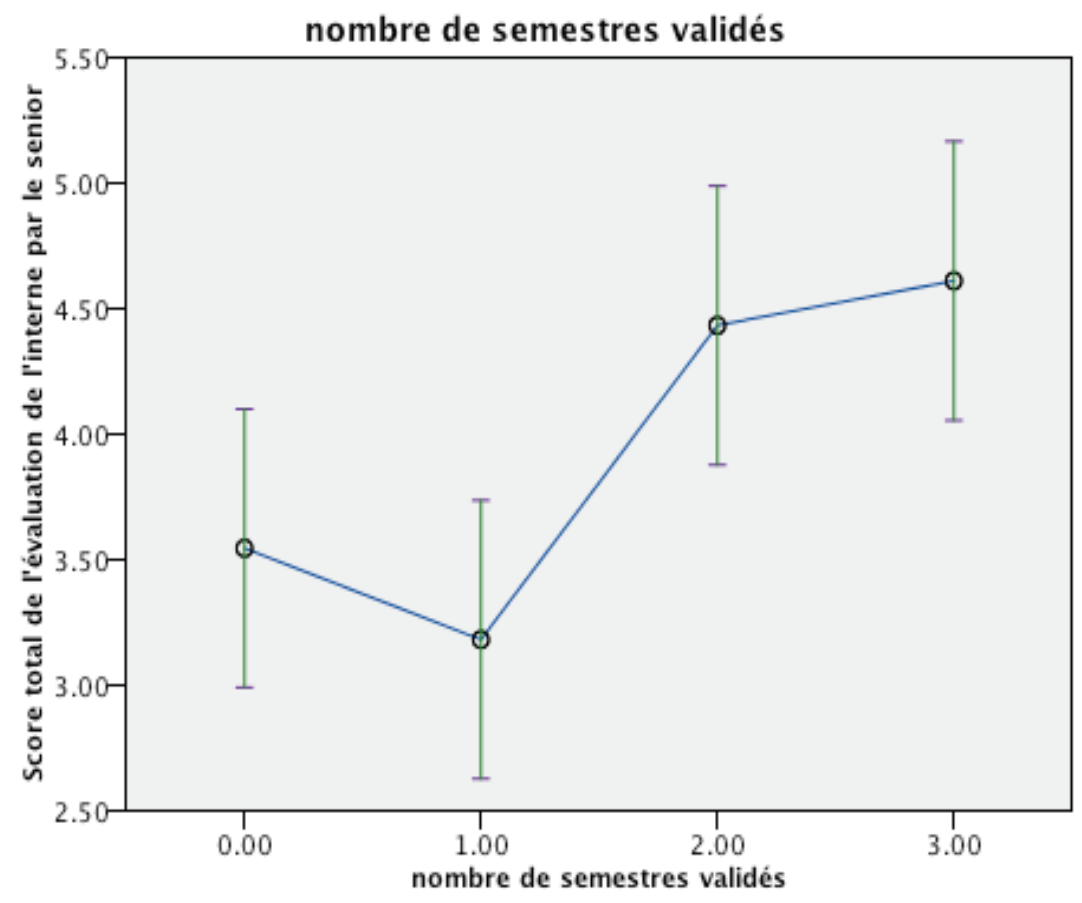

Figure 23 : Evolution des scores totaux des internes évalués par les obstétriciens de garde en fonction de l'ancienneté des internes.

Les internes qui étaient considérés comme apte à réaliser une extraction par spatules de Thierry avec supervision indirecte avaient validé en moyenne 2,8 semestres contre 2,1 pour ceux qui avaient besoin d'une supervision directe et 0,9 semestre pour les internes qui ne pouvaient qu'observer ou assister $(\mathrm{p}=0,003)$ [Figure 24]. 


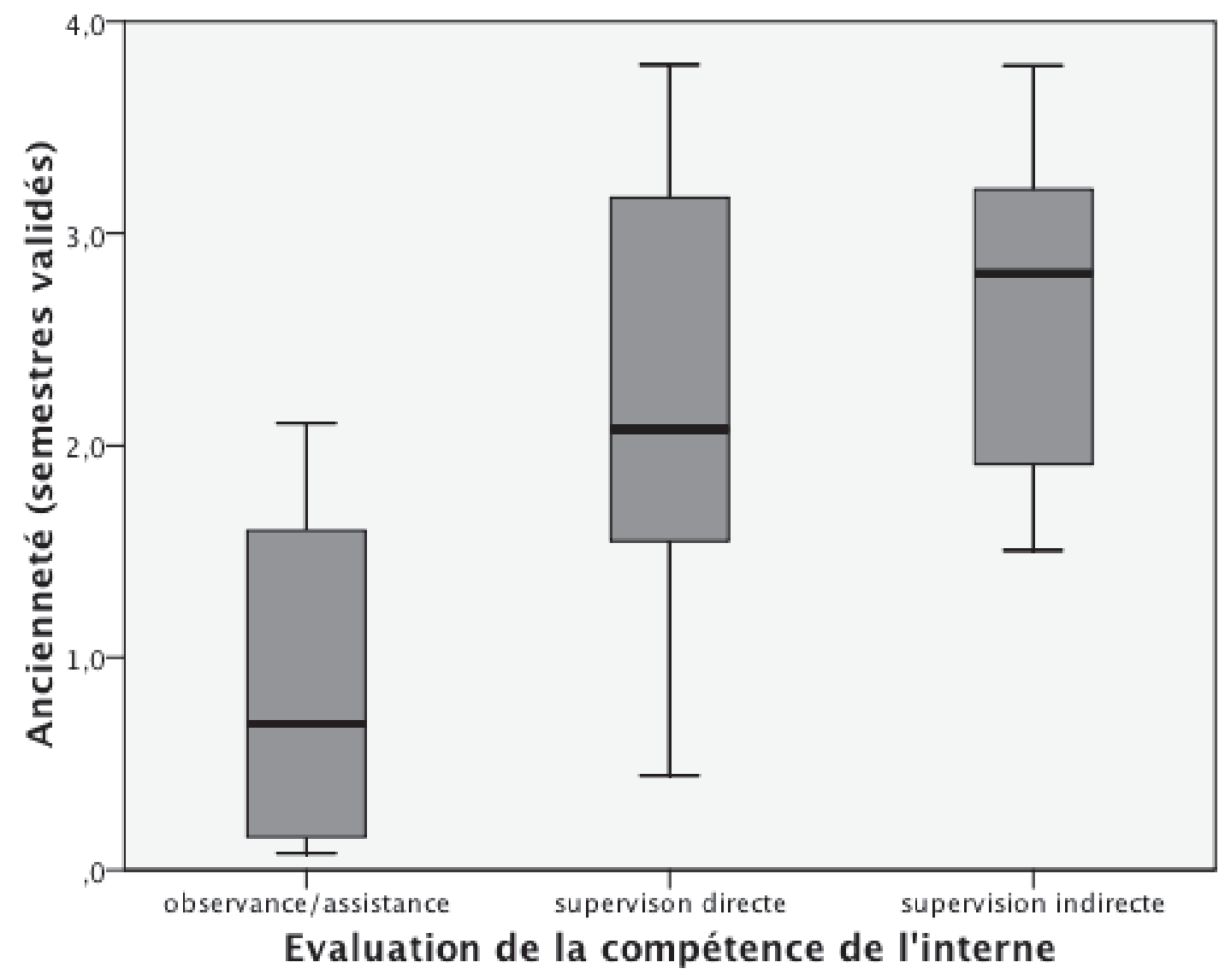

Figure 24 : Niveau de compétence globale de l'interne selon l'obstétricien de garde.

Le niveau de confiance des internes tendait aussi à augmenter avec leur ancienneté (figure $7 ; p=0,06$ ) [Figure 25]. 


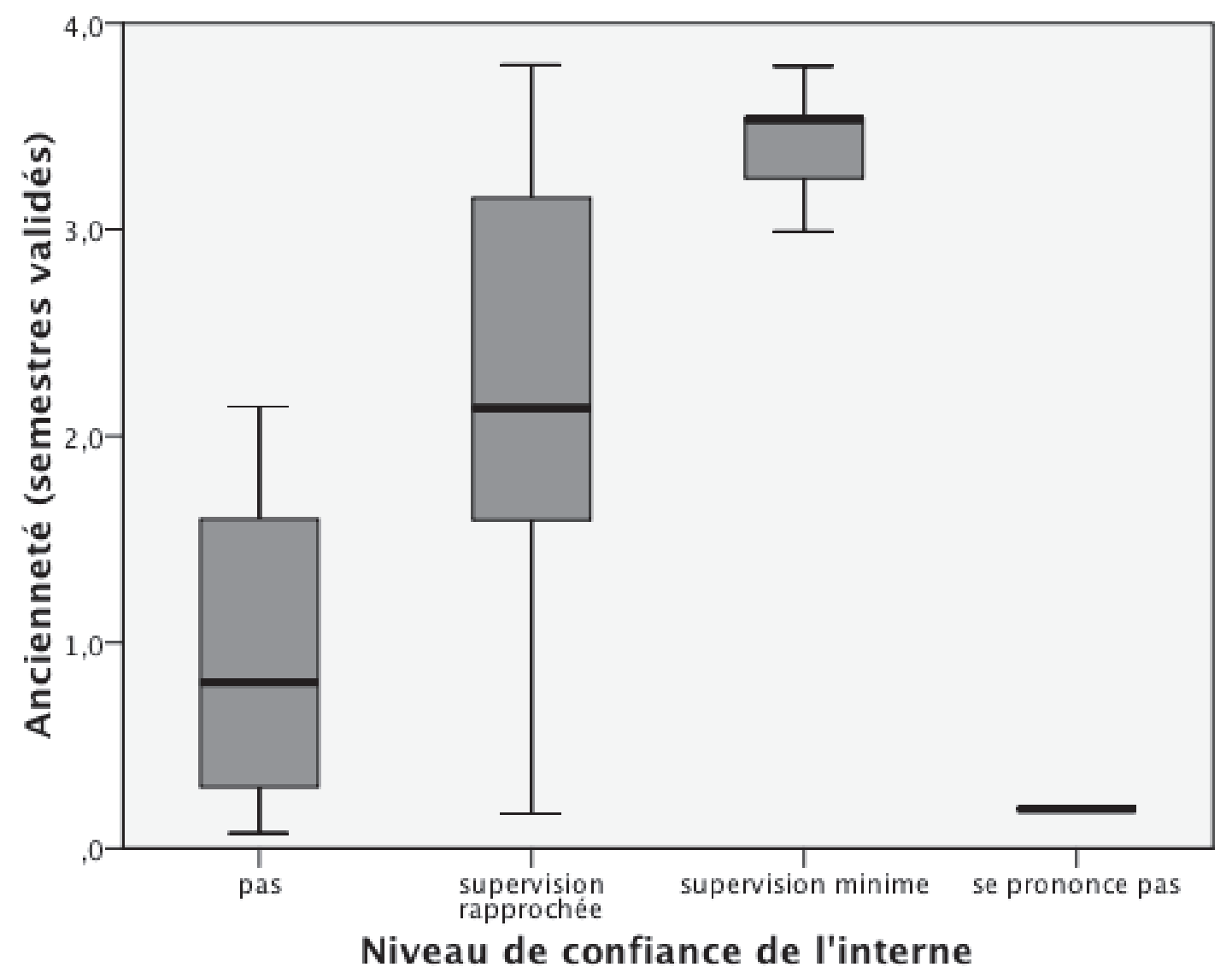

Figure 25 : Répartition du niveau de confiance de l'interne en fonction de son ancienneté.

\section{2) Impact du niveau d'expérience des internes sur leurs résultats aux évaluations}

Lorsqu'on étudie les extractions réalisées par les 4 internes suivis depuis le début de leur formation, dans $24 \%$ des cas l'interne avait participé à plus de 20 extractions par spatules avant l'extraction évaluée. Selon le MLM, le score global moyen était significativement supérieur lorsque l'interne avait participé à plus de 20 extractions avant l'extraction étudiée (4,0 vs 3,7; p<0,001) [Figure 26] .Le fait d'avoir réalisé plus de 20 extractions était statistiquement associé au fait d'être reconnu par le médecin sénior comme capable de réaliser une extraction sous supervision directe ou indirecte $(100 \%$ vs $68 \%, \mathrm{p}=0,031)$. 
Aucun interne qui avait participé à plus de 20 accouchements assistés par spatules de Thierry n'était considérait apte à réaliser des extractions instrumentales par spatules sans supervision.

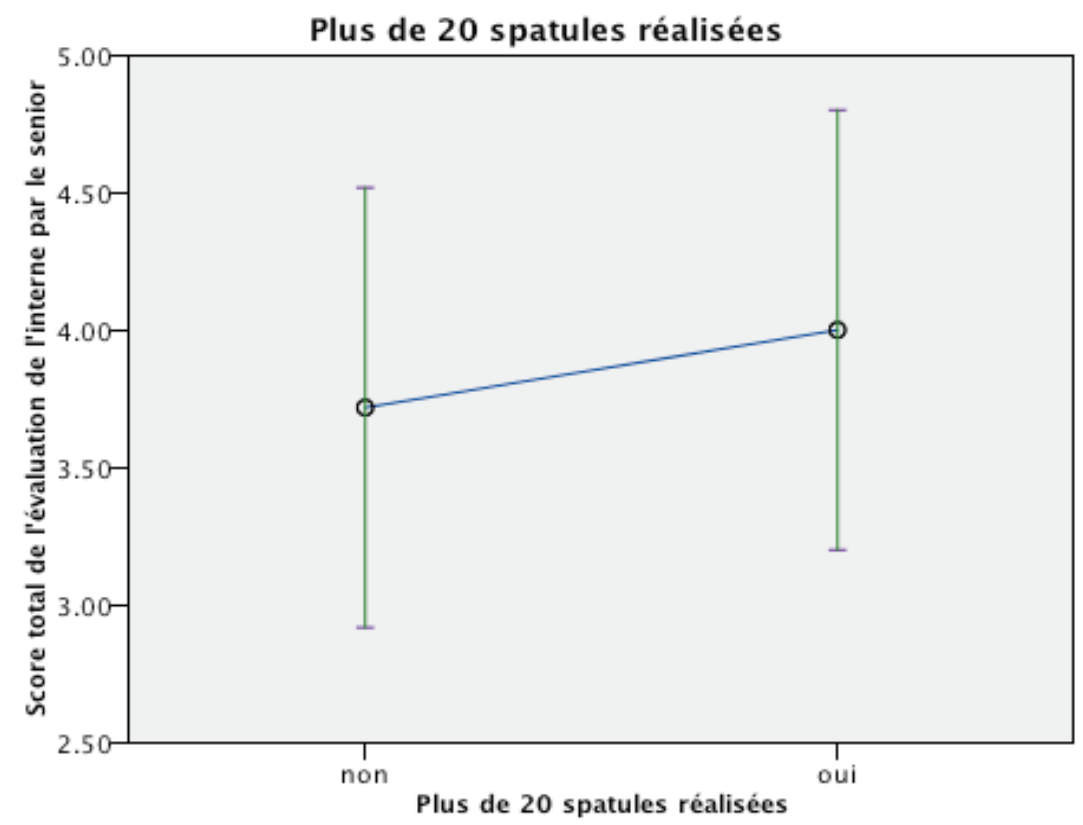

Figure 26 : Répartition des scores de l'évaluation des internes par le senior en fonction du seuil de réalisation des 20 extractions par spatules.

3) Niveau de confiance de l'interne en fonction de l'ancienneté et du seuil des 20 extractions réalisées

Le niveau de confiance des internes tendait aussi à augmenter avec leur ancienneté (figure 7 ; $\mathrm{p}=0,06)$ [Figure 25].

Les internes qui avaient réalisés plus de 20 extractions se sentaient plus fréquemment en confiance au moment de l'accouchement $(100 \%$ vs $68 \%, \mathrm{p}=0,034)$, tout en souhaitant toujours bénéficier d'une supervision rapprochée par l'ODG.

Une sensation de progression dans l'acquisition du geste était ressentie chez les internes qu'ils aient ou non réalisé plus de 20 extractions (92\% vs 92\% p=1,000). 


\section{V-DISCUSSION}

\section{A- POINTS FORTS ET LIMITES DE L'ÉTUDE}

Ce travail prospectif est le premier à s'intéresser à la courbe d'apprentissage des extractions par spatules de Thierry en utilisant une grille d'évaluation systématique validée. Les différents items de la grille permettent d'appréhender exhaustivement la progression des internes dans différents domaines. Un autre point fort de notre étude est le recueil prospectif des données cliniques sur chaque accouchement vaginal assisté par ST, qui ont permis de témoigner de l'activité de notre service et de l'exposition clinique des internes.

Les évaluations n'ayant pas été remplies immédiatement au cours de l'extraction ont été exclues afin de ne pas biaiser, par une évaluation devenue rétrospective, la validité de l'analyse. L'application stricte de ces critères d'analyse associée aux contraintes liées à une activité de garde en salle de naissance explique le faible nombre de grilles d'évaluation recueillies au cours de notre étude. Néanmoins, l'absence de différences statistiquement significative entre les caractéristiques des accouchements ayant fait l'objet d'une évaluation et celles des accouchements exclus témoigne de la représentativité des évaluations étudiées, et rassure sur l'absence de biais de sélection.

Le faible effectif de ce travail original est le reflet de la difficulté de mener une étude prospective basée sur une participation active et bénévole dans un CHU de niveau III. Malgré la motivation des internes participants et des ODG, les évaluations n'ont pas été réalisées aussi régulièrement que prévues. Un retour régulier et individualisé sur les taux d'évaluations des personnes participantes (par courrier, affiche dans le service ou communication orale lors des réunions internes) aurait pu augmenter la régularité d'évaluation. Un système de dédommagement des participants serait une solution à proposer aurait pu être une autre solution. D'autre part, l'étude de la progression des internes dans la 2ème phase de notre étude n'était peut-être pas représentatif de tous les internes en début d'apprentissage, car certains d'entre eux avaient déjà une expérience obstétricale préliminaire dans les extractions par spatules de Thierry. Ces données ont pu influencer directement leur rapidité 
d'apprentissage au cours de l'étude, mais leur ancienneté a été prise en compte dans nos calculs.

Un autre biais potentiel réside dans le fait que les internes se savaient évalués par l'ODG. Même si en dehors de notre étude les internes sont évalués de manière continue, le caractère explicite de l'évaluation du fait du protocole de notre étude a pu causer un stress chez les internes et diminuer leurs performances.

De plus, la qualité de l'interaction entre l'interne et l'ODG pouvait biaiser l'évaluation. Dans la première partie de notre étude, il n'y avait pas d'ODG qui se démarquait des autres en terme de score total moyen. Le faible nombre d'évaluation dans la seconde partie de notre étude ne permet malheureusement pas d'évaluer si certain ODG sous ou surévaluait systématiquement.

\section{B - INTERPRÉTATION DES RÉSULTATS DE LA PREMIERE PHASE DE NOTRE ÉTUDE}

\section{1) Comment valider notre outil d'évaluation}

Compte tenu de l'importance de la formation des internes, l'évaluation de celle-ci est indispensable. Cette évaluation peut concerner à la fois les apprenants et les formateurs. En médecine, les échelles d'évaluation sont utilisées dans de nombreux travaux, et sont composées de plusieurs items dont la cotation est combinée en un score global ou des sous scores dimensionnels [96].

Dans d'autres domaines elles permettent d'évaluer des phénomènes subjectifs ou complexes tels que la douleur, la qualité de vie, le handicap, etc.

Pour être fiables, ces grilles d'évaluation doivent posséder les qualités d'un bon instrument de mesure [Annexe 5] :

- validité

- fidélité

- cohérence interne

- sensibilité du changement.

Notre étude a analysé chacune de ces qualités dans le but de valider la grille d'évaluation des extractions par spatules de Thierry. 


\section{Validité de contenu}

La grille d'évaluation a été construite sur le modèle d'un audit clinique et sur la base des objectifs de la formation aux extractions instrumentales décrits par Dupuis [85], Sentilhes et al. [24] et de la grille d'évaluation des accouchements instrumentaux du RCOG [26]. Les items des la grille représentent toutes les étapes des accouchements vaginaux assistés par spatules de Thierry.

Sa validité de contenu a été confirmée par 3 praticiens experts, exerçant actuellement et ayant chacun au moins 10 ans d'expérience des accouchements assistés par spatules au moment de l'étude.

\section{Validité contre critère}

Il n'existe pas d'échelle de référence à laquelle comparer notre grille d'évaluation des extractions par spatules de Thierry; on peut cependant vérifier la corrélation entre les complications maternelles (déchirure périnéale sévère, déchirure vaginale sévère), le score de l'interne et le niveau de difficulté de l'extraction.

$\checkmark \quad$ Les scores totaux et les niveaux de compétence globale ne différait pas significativement en cas de lesion perineale sévère [Tableau 9, Figure 22]. On note tout de même des scores inférieurs dans l'item «protection du périnée ». Ces lésions périnéales sévères survenaient essentiellement quand l'interne considérait une extraction instrumentale difficile. [Tableau 8]

\footnotetext{
$\checkmark$ Le taux de déchirures vaginales des internes «junior» était corrélé à la survenue d'extractions jugées difficiles.

$\checkmark \quad$ La corrélation entre les complications néonatales, le score de l'interne et le niveau de difficulté n'a pu être vérifiée du fait du faible taux de complications néonatales.
}

Certes notre outil d'évaluation ne vérifie pas la corrélation entre les complications maternelles des extractions par spatules, le score de l'interne et le niveau de difficulté de l'extraction, mais des tendances sont émises, en l'absence d'outil de référence.

\section{$\underline{\text { La validité de construit }}$}

Le but de notre échelle étant d'évaluer les performances des internes lors des extractions par spatules, nous avons vérifié les hypothèses suivantes : 
$\checkmark \quad$ plus l'interne a validé de semestres meilleures est son score total: les internes « seniors » avaient tendance à avoir un meilleur score total que les « juniors » (mediane 4,7 versus 4,$3 ; \mathrm{p}=0,068)$.

$\checkmark \quad$ plus l'interne a validé de semestres plus le senior le juge autonome : après $64 \%$ des extractions réalisées par des internes «seniors » l'ODG considérait l'interne capable de réaliser une extraction sans supervision, alors qu'il ne portait cette appréciation que dans $9 \%$ des cas lorsque l'interne était « junior » $(\mathrm{p}=0,005)$.

$\checkmark \quad$ Plus l'interne a un score élevé plus l'ODG le juge capable d'autonomie : Les internes jugés capable de réaliser une extraction sans supervision avaient des scores globaux supérieurs aux autres (médiane 4,9 versus 4,2; $<<0,001$ ).

La validité de construit de notre échelle est globalement vérifiée, mais d'autres études devront confirmer les hypothèses précédentes.

\section{Fidélité}

La fidélité inter juge : le score de l'échelle d'évaluation d'un interne ne dépend pas de qui est son évaluateur ; les différents évaluateurs doivent avoir le même avis concernant la capacité de l'interne à être autonome.

$\checkmark \quad$ La fidélité test-retest : l'interne et son évaluateur doivent avoir le même jugement concernant la capacité de l'interne à être autonome.

$\checkmark \quad$ La fidélité inter-juge de la grille d'évaluation des extractions par ST n'a pas pu être vérifiée ; cela aurait nécessité la présence de 2 ODG évaluateur dans la salle d'accouchement au même moment, ce qui était difficile à mettre en place au vu de l'organisation de notre service et du caractère urgent de la plupart des extractions.

L'absence de différence dans les scores totaux moyens émis par les 2 ODG qui ont participé au plus grand nombre d'évaluation est rassurance quant à l'absence de sur ou sous évaluation systématique. La fidélité de la grille d'évaluation sera à confirmer dans un prochain travail.

\section{Cohérence interne}

$\checkmark \quad$ Le coefficient alpha de Cronbach de la grille d'évaluation est de 0,99 , ce qui témoigne d'un haut degré de corrélation entre les résultats de chaque item, et vérifie que l'ensemble de 
la grille d'évaluation correspond à une dimension clinique unique.

La sensibilité au changement n'a pu être vérifiée dans la première partie de notre étude, mais fait l'objet de la seconde.

Actuellement il n'existe pas d'autre outil d'évaluation des extractions par ST que la grille qui a été mise au point dans ce travail.

Certes la grille d'évaluation ne vérifie pas statistiquement toutes les qualités d'un bon instrument de mesure, mais il existe tout de même des tendances qui supportent sa pertinence. D'autres études devront confirmer la validation de cet outil d'évaluation sur des cohortes plus importantes de médecins en formation.

\section{2) Les complications maternelles}

Les complications maternelles sévères de notre étude incluaient les lésions périnéales du 3ème degré et plus, les hémorragies de la délivrance et les thrombus vaginaux. Le taux de ces complications était globalement identique que l'interne ait été «junior» ou «senior» et tendait à être plus important quand l'extraction était considérée comme difficile. De plus on note un taux de révision utérine supérieur chez l'interne plus jeune sans qu'il existe une corrélation avec le taux d'hémorragie de la délivrance. Notre hypothèse est que la réalisation d'une révision utérine permettait à l'équipe médicale de suturer la déchirure périnéale immédiatement et de limiter le saignement d'origine vaginale, souvent important après une extraction difficile.

Les résultats d'une étude précédente menée dans le service ont montré un sur-risque de lésion périnéale sévère maternelle lorsque l'extraction était réalisée par un interne peu expérimenté, par rapport à un interne de plus de cinq semestres $(8,0 \%$ versus $4,5 \% \mathrm{p}=0,035 ; \mathrm{OR}=2,10$ IC95\% [1,16-3,77])[12]. Cependant le caractère rétrospectif de cette étude ne permettait pas de connaître la proportion du geste effectué par chaque interne et était biaisée par la potentielle sous déclaration des complications par les internes « seniors ».

Dans notre étude, le taux de lésions périnéales sévère avait tendance à être plus élevé lorsque les extractions étaient réalisées par l'interne «junior» comparé à l'interne « senior », sans que cette différence soit significative [ Figure 22] ; ces complications correspondaient 
d'ailleurs à des évaluations inférieures dans l'item «protection du périnée ». La présence quasi-systématique de l'obstétricien de garde lors des extractions étant une garantie de l'absence de biais de déclaration, cette trouvaille confirme la nécessité d'une vigilance accrue lors de la supervision des internes en début de cursus. Il peut exister une perte de chance pour la mère au début de l'apprentissage des extractions par ST qui impose la présence de l'ODG à chaque extraction instrumentale. C'est un argument pour poursuivre le développement d'outil de simulation, permettant aux internes de débuter leur apprentissage sans risque pour les patientes.

On note de plus une tendance à moins de dégagement en OS dans les cas d'extractions par ST évaluées, ce qui pourrait refléter la propension des ODG à réaliser eux-mêmes les extractions dans ces conditions où l'accommodation foeto-pelvienne est moins bonne et où le risque de déchirures périnéales sévères est supérieur ; en effet il a été démontré que lors des extractions par ST, le risque de lésion du sphincter anal est significativement augmenté en cas de dégagement en OS avec un OR de 6,4 (95\% IC 1,3-31,5) par rapport à un dégagement en OP [13]. Ces résultats pourraient laisser à penser que s'il existe un sur risque de lésion périnéale sévère lorsque l'extraction est réalisée par un interne «junior», il est partiellement contrôlé par l'encadrement des internes et la sélection de cas à leur porté.

Notre travail démontre l'importance de la notion d'ancienneté de l'interne qui doit être prise en compte, notamment dans le risque périnéal maternel qu'impliquent les extractions par ST.

\section{3) Les complications fotales}

Sur le plan fœtal, notre étude a retrouvé très peu de complications attribuables aux extractions par ST. Ce résultat corrobore les trouvailles d'études de cohorte rétrospectives récentes [13,8]. Ainsi dans notre service, entre 2003 et 2006, nous avions observés $0.7 \%$ de complications orthopédiques (fracture de la clavicule et humérus), 0,3\% de paralysie du nerf facial, $0,3 \%$ de lésion du plexus brachial et $0,7 \%$ de cephalhematome [3].

De même, Mazouni et al., dans une série marseillaise de 279 cas ne rapporte aucune complication néonatale attribuable aux extractions par ST [8]. 
Des publications plus anciennes ne décrivent aucune lésion néonatale grave malgré l'inclusion d'extractions par ST de nouveau-nés de moins de 37 semaines d'aménorrhée, pourtant considérés comme plus fragiles [74, 97,98].

\section{C - INTERPRÉTATION DES RÉSULTATS DE LA 2ÈME PHASE DE NOTRE ÉTUDE}

\section{1) L'intérêt des courbes d'apprentissage en obstétrique}

Les extractions fœtales instrumentales nécessitent une maitrise du geste en raison des complications materno-fœtales qu'elles sont susceptibles d'engendrer [8].

Si le choix de l'instrument au moment du geste est dicté par des indications obstétricales, il reste souvent affaire de choix personnel ou d'école obstétricale.

La formation aux extractions instrumentales pendant l'internat de GO a pour objectif d'assurer une sécurité maternelle et fotale optimale [85].

Pourtant, à ce jour peu d'études se sont intéressées à leurs courbes spécifiques d'apprentissage et le nombres seuil d'EI qu'il faudrait avoir réalisé avant de pouvoir les pratiquer de manière autonome demeure indéterminé.

La formation actuelle de l'interne aux extractions par spatules est basée sur le compagnonnage, au lit de la patiente, qui a ses limites, car il s'effectue souvent dans un contexte d'urgence, et dans une atmosphère où le risque médico-légal est souligné [85].

Même s'il n'existe pas d'obligation de résultat face à un accouchement pathologique, il y a cependant une obligation de moyen, ce qui impose un encadrement par des professionnels compétents pour augmenter la qualité et la sécurité de l'extraction [99].

La validation de courbes d'apprentissage pour chaque instrument d'extraction serait une référence pour tester l'évolution des performances de chaque gynécologue obstétricien en formation. 


\section{2) Les courbes d'apprentissage retrouvées dans la littérature}

Aucune donnée dans la littérature ne concerne la forme des courbes d'apprentissage dans les différents types d'EI.

\section{Un modèle expérimental}

Une ancienne étude expérimentale décrivait la forme caractéristique des courbes d'apprentissage d'un geste technique. Il s'agissait d'établir un schéma théorique rendant compte du mécanisme de l'apprentissage et du rôle que jouent les différents éléments de la tâche [100] [Figure 27].

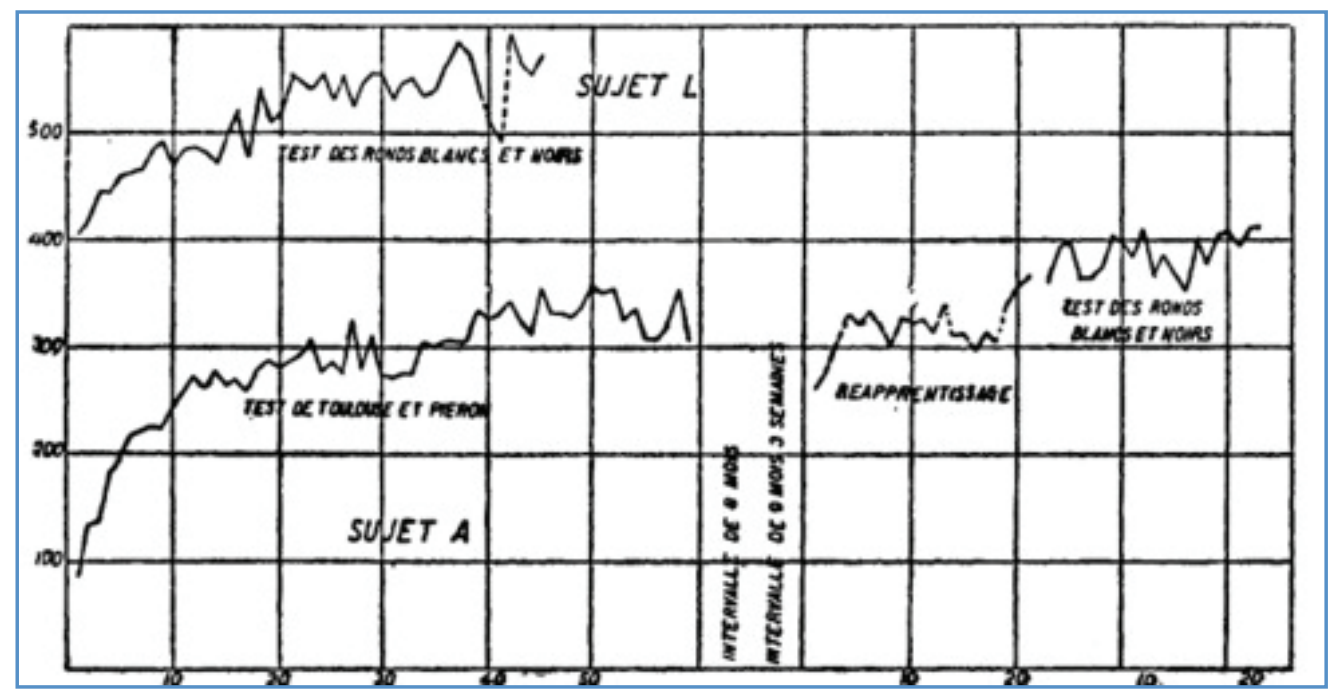

Figure 27 : Schéma théorique des courbes d'apprentissage

La forme de cette courbe expérimentale correspond à une montée rapide et régulière au début, puis un ralentissement et enfin une montée lente.

\section{Un modèle en anesthésie réanimation}

Un autre modèle retrouvé dans la littérature concernait la courbe d'apprentissage théorique de l'anesthésie locorégionale chez nos confrères médecins anesthésistes réanimateurs. On observe une variation de la forme de cette courbe en fonction de l'opérateur et ses connaissances, son expérience et sa dextérité [101]. 
Trois phases distinctes dans l'apprentissage peuvent être soulignées :

$\checkmark$ initiation

$\checkmark$ perfectionnement

$\checkmark$ expertise.

À chaque étape correspond un apprentissage différent :

$\checkmark$ qu'il soit théorique : cours magistraux, diplôme interuniversitaire, support audiovisuel, participation à des congrès de la spécialité ou

$\checkmark$ pratique : stage à l'hôpital, formation pratique, nombre précis de geste à réaliser.

[Figure 28]

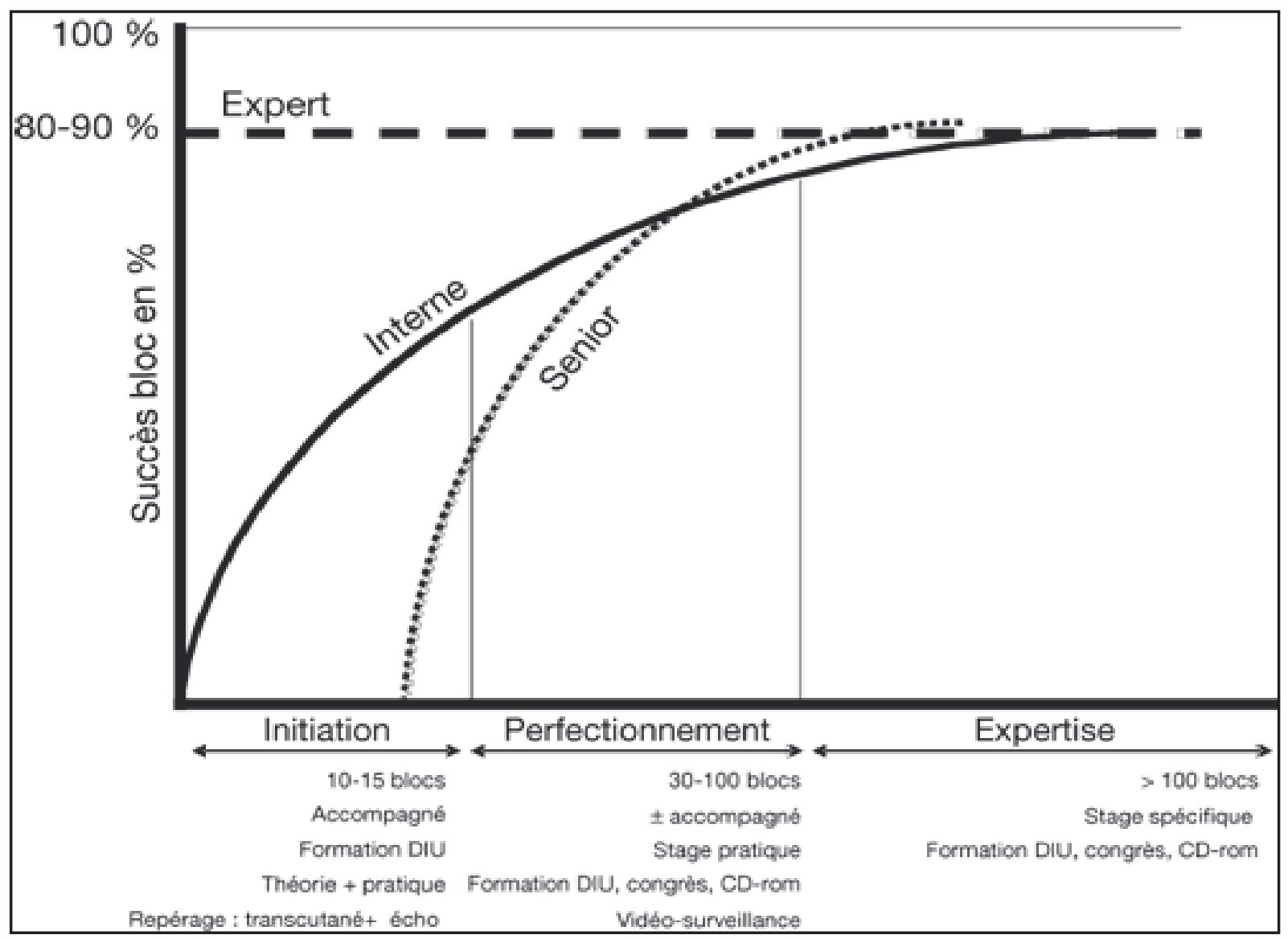

Figure 28: Courbes d'apprentissage théorique de l'anesthésie locorégionale en fonction du degré de formation préalable et des moyens utiles à ce stade. 


\section{3) La courbe d'apprentissage des extractions par forceps}

Selon une étude sur simulateur d'extraction par forceps, 31 poses de cet instrument sont nécessaires avant que l'étudiant n'en maitrise la pratique pour les présentations OP (NP4) [102]. Les variétés obliques nécessiteraient probablement un minimum de 62 forceps avant d'être jugé compétent (avis d'expert) [102].

De grandes différences dans la vitesse d'apprentissage entre les étudiant a pu être observée : pour la seule pose en variété OP, au moins 7 forceps doivent être réalisés pour assurer une pose adéquate de la moitié des étudiants [102].

\section{4) La courbe d'apprentissage des extractions par ventouse}

Dans un travail récent sur la courbe d'apprentissage de la ventouse, une progression significative des internes en début de cursus sur une dizaine de procédure avait été observée, cependant le nombre de procédures à réaliser avant d'être considéré “autonome" n'avait pu être déterminé [103].

\section{5) Combien d'extractions faut-il réaliser pendant l'internat de gynécologie obstétrique?}

Peu de recommandations concernent le nombre d'EI qu'il faut avoir réalisé avant de passer à la phase d'autonomie.

L'EBCOG (European Board and College of Obstetricians and Gynaecologists) recommande 40 extractions en comptant ventouse, forceps et siège (EBCOG 18/03/2008) [15].

Ces recommandations exclue les extractions par ST, probablement du fait que leurs emplois restent limités à la francophonie et à quelques pays hispaniques (Espagne et Amérique du sud), raison autre pour laquelle peu de données existent dans la littérature à ce sujet $[5,6]$.

Une enquête réalisée en 2004 chez les internes d'obstétrique de 38 états américains a montré qu'en fin d'internat, $80 \%$ d'entre eux avaient réalisé moins de 40 forceps et de 40 ventouses, et à peine $50 \%$ se sentaient compétents pour réaliser des forceps ; la plupart considéraient 
qu'il leur manquait la réalisation de 20 forceps pour être compétents [92]. Concernant les variétés obliques nécessitant un apprentissage spécifique, ce sont probablement un minimum de 62 forceps qu'il faudrait avoir réalisés (avis d'expert) [85].

Certains auteurs ont tenté de vérifier si ce nombre d'extractions par ventouse et forceps, était réalisable dans un établissement de 3000 accouchements par an qui disposerait de 10 internes en 1 an, en émettant des hypothèses statistiques sur la répartition de ces extractions et leurs indications ; la durée du DES de GO ne permettait pas d'atteindre ce seuil de 62 forceps par interne.

Ces hypothèses n'intéressaient pas les extractions par ST [85].

\section{6) La courbe d'apprentissage des extractions par spatules de Thierry}

Les spatules de Thierry ont la réputation d'avoir une courbe d'apprentissage rapide, sans qu'aucune donnée n'ait été publiée sur ce sujet avant notre travail.

En 1964, LLyod avait proposé un seuil 20 procédures d'extractions par spatules de Thierry pour être jugé compétent [17]. Le nombre proposé ne se basait sur aucune évaluation objective de l'extraction effectuée; les critères qui définissaient de la compétence d'un obstétricien sur les extractions par ST n'étaient pas mentionnés. L'auteur basait son analyse en comparant l'instrument conventionnel à l'époque, le forceps, et un nouvel instrument les ST ; les différents items de comparaison concernaient la durée du travail, la difficulté de l'extraction, le type de rotation de la présentation effectué, les complications materno-fœtales. L'auteur concluait à la rapidité d'apprentissage de cet instrument et au nombre de 20 procédures nécessaires avant de pouvoir le maîtriser.

Dans notre étude, les ODG ont objectivement jugé, par utilisation de la grille d'évaluation validée, qu'aucun interne n'était apte à une extraction autonome au bout de 20 procédures. De même, de leur côté et de manière indépendante, aucun interne ne s'est jugé en complète confiance pour la réalisation de toutes les extractions par ST après la participation à 20 extractions. Il est à noter que ni les médecins ni les internes n'étaient informés du choix du seuil de 20 extractions pour notre étude. Nos résultats montrent que le nombre de 20 extractions est nécessaire pour obtenir une amélioration significative des scores d'évaluation 
et permettre à l'interne d'être jugé suffisamment compétent pour profiter des effets d'une supervision par l'ODG dans un cadre clinique.

La courbe d'évolution des scores totaux des internes en fonction de leur ancienneté issue de notre travail peut être interprétée en 3 phases. La première phase, une progression lente, correspond à la période initiale d'assimilation des étapes de la technique sur le plan théorique. La seconde phase, marquée par une ascension nette, correspond à une phase d'acquisition des connaissances pratiques avec coexistence des connaissances théoriques. Enfin, la troisième phase voit apparaître une nouvelle ascension plus lente, les bénéfices de l'expérience pratique permettant un perfectionnement dans le geste d'extraction. Cet aspect est typique de l'apprentissage d'un exercice donné [100] [Figure 27].

\section{7) L'intérêt des simulateurs obstétricaux pour améliorer la courbes d'apprentissage des extractions instrumentales}

L'enseignement par compagnonnage au lit de la patiente, essentiel dans la phase de perfectionnement et de "mise en responsabilité" de l'interne, semble d'un intérêt limité dans la phase initiale d'apprentissage des EI. En effet cette phase nécessite du temps et la décomposition du geste technique, ce que le contexte d'urgence ne permet pas souvent d'offrir.

Les simulateurs obstétricaux sont un outil d'apprentissage qui devient indispensable puisqu'ils permettent une pratique objectivement évaluable, jusqu'à atteindre les seuils de pratique, en sécurité, des EI [104].

Ils permettent aux professionnels de s'entrainer sans danger à la pratique des accouchements normaux et pathologique tels que les EI, et notamment de simuler des situations cliniques rares.

L'addition à la formation traditionnelle d'une formation par simulation est un atout majeur pour la formation à l'EI. Cependant la littérature sur ce sujet est encore limitée.

Différents modèles de simulateurs d'extractions par forceps ont été décrits mais il n'existe pas à ce jour de modèle virtuel de simulateur obstétrical d'extraction par ST. 
Aucune étude ne permet actuellement de connaître avec précision le nombre d'EI souhaitable; les simulateurs obstétricaux rendraient cet objectif envisageable. Le nombre minimum d'EI à réaliser pendant l'internat est probablement voisin de 60 pour les forceps (avis d'expert), de 10 pour la ventouse obstétricale [103] et de plus de 20 pour les ST d'après les résultats de notre étude.

Les simulateurs obstétricaux permettraient probablement d'améliorer la vitesse d'apprentissage des EI par ST, comme cela a été démontré spécifiquement dans les EI par forceps [102,104] [Figure 29].

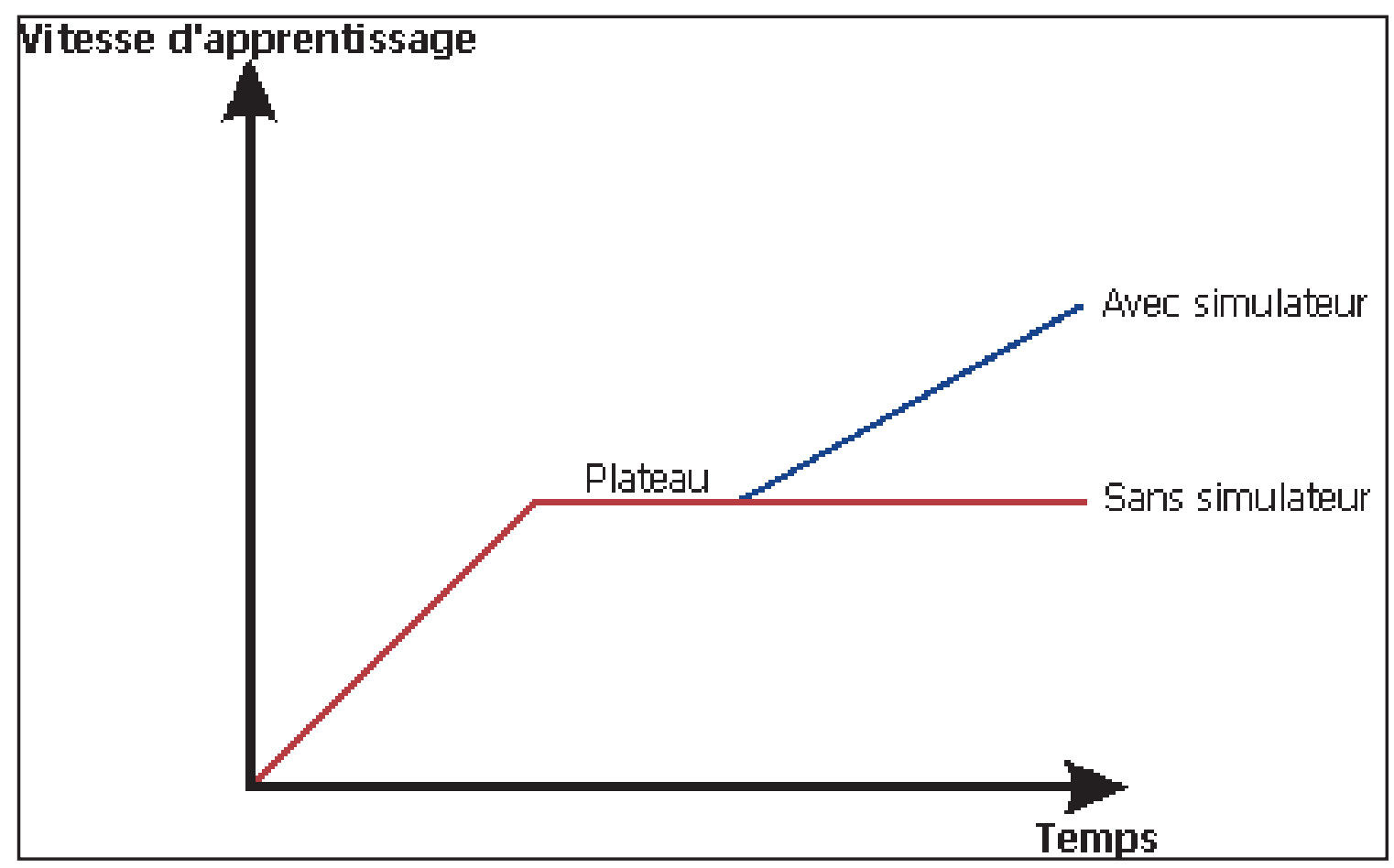

Figure 29 : Variation de la vitesse d'apprentissage après mise en situation sur simulateurs. 


\section{D - EVALUATION DE LA FORMATION DES INTERNES EN GYNECOLOGIE OBSTETRIQUE}

\section{1) Evaluation des compétences de l'interne de gynécologie obstétrique}

En France, le livret de l'interne a été créé dans le but d'évaluer les compétences de l'interne et de noter le nombre de procédures réalisées en GO lors des stages. Il reste tout de même sous utilisé et n'indique pas le nombre de gestes nécessaires à réaliser pour être considéré comme compétent, notamment pour les extractions instrumentales [105].

L'apprentissage et l'évaluation objective des performances de l'interne aux extractions sur simulateur et dans une mise en situation réelle devraient faire partie intégrante de son cursus de GO [104]. Ladite évaluation représente un outil complémentaire pour leurs progressions mais également déterminer une éventuelle qualité ou faiblesse d'un enseignement

L'objectif d'une formation reste le couplage d'un enseignement théorique avec une pratique en situation, jusqu'à l'obtention de l'autonomie de l'étudiant.

Ce carnet de bord de l'internat de GO devrait être revu afin d'inclure des évaluations de l'interne sur simulateur en gynécologie et en obstétrique.

D'une manière générale, l'évaluation objective des internes en GO est presque inexistante : dans une enquête nationale, les gynécologues obstétriciens interrogés sur la formation chirurgicale des internes ont confirmé l'absence d'évaluation formative sur la base de critères explicites [106]. Pourtant le domaine de la formation chirurgicale offre des modèle validés et complets [107], et applicable à la GO [108,109].

L'objectif de cette enquête intéressait uniquement l'exploration des différentes facettes de l'enseignement chirurgical. Des modèles d'évaluations des compétences des internes appliqués à l'obstétrique devront faire l'objet d'études complémentaires, plus spécifiquement dans l'évaluation des EI et spécifiquement par ST. 


\section{2) Les objectifs de la formation aux extractions instrumentales}

Les Objectifs de la formation aux extractions instrumentales ont été rappelés par Dupuis [85].

\section{Les objectifs sont les suivants :}

1. permettre au médecin de ne pas recourir systématiquement à la césarienne en cas de dystocie ou en cas d'anomalies significatives du rythme cardiaque fœtal ;

2. savoir poser les indications ;

3. connaître les contre-indications ;

4. connaître les pré requis (état maternel, état fœtal, asepsie, collaboration sage femme / obstétricien / anesthésiste) ;

5. savoir choisir l'instrument ;

6. effectuer correctement les poses de ventouse, de forceps et de spatules, afin de minimiser les risques d'échec de pose ;

7. effectuer correctement les tractions avec ventouse et forceps (direction de la force, nombre d'effort), afin de minimiser les risques d'échec de traction ;

8. connaître les éléments qui doivent faire abandonner une EI en cours ;

9. savoir rédiger un compte rendu d'extraction instrumentale (facilité de la pose, nombre de traction, pour les ventouses nombre de détachement, état périnéal, état néonatal);

10. minimiser le recours à l'utilisation séquentielle des instruments ;

11. savoir dépister et prendre en charge les complications périnéales et les hémorragies de la délivrance secondaires aux EI ;

12. assurer le suivi maternel des éventuelles complications ;

13. connaître les principes de l'autocontrôle qualité (tenue d'un livre de bord et du nombre d'échecs d'extraction instrumentale, fréquence des complications néonatales). 


\section{3) De quels moyens dispose-t-on pour former l'interne en gynécologie obstétrique aux extractions instrumentales?}

\section{Formation au savoir « factuel »}

La réalisation d'EI nécessite l'acquisition d'un savoir comprenant de nombreuses connaissances dont :

- l'anatomie de la mère et du fotus

- la mécanique obstétricale et sa physiologie

- l'instrument d'extraction : ses principaux modèles, la technique de pose, la technique du geste d'extraction (traction/propulsion) et d'orientation

- connaître les principales complications des extractions instrumentales

\section{Formation au savoir « conceptuel »}

La réalisation d'EI nécessite la connaissance de plusieurs concepts liés à la sécurité. Ces concepts concernent :

- les rapports entre le fœtus et le canal pelvien : la hauteur de la tête fœtale, le degré estimé d'une éventuelle disproportion foeto pelvienne, la synchronisation des forces mécaniques

- la communication entre sage femme, anesthésiste réanimateur, obstétricien

- le contrôle qualité : démarches d'évaluation des pratiques professionnelles, la relève annuelle du nombre d'EI, le taux d'échec de la pose, le taux d'échec de la traction, le nombre de complications maternofoetales.

\section{Formation au savoir « technique »}

L'enseignement de ces techniques est réalisé au moyen d'une mise en situation réelle par compagnonnage ou par le biais d'un apprentissage par simulateur.

Les simulateurs obstétricaux sont un outil d'apprentissage qui deviendra rapidement incontournable, nécessaire à la formation de l'interne. Ils permettront aux internes de réaliser une partie de leur apprentissage sur mannequin [104]. 
Actuellement les simulateurs obstétricaux intéressent les forceps [102] et ventouses mais sont inexistants pour les extractions par spatules de Thierry, probablement lié à l'inhomogénéité d'utilisation ce type d'extraction dans le monde.

La qualité d'un simulateur aux extractions peut reposer sur trois aspects à savoir l'interface mécanique, l'interface de visualisation et la boucle retour de la commande. Le modèle BirthSIM les exploite [110] [Figure 30] :

- Une partie mécanique automatisée composée de mannequins anthropomorphes (bassin maternel, tête fœtale et muscles pelviens)

- Une partie électropneumatique qui permet de reproduire les différents efforts mis en jeu (contractions utérines, efforts de poussées) et d'un système rotatif qui permet de positionner la présentation fœtale

- Une interface de visualisation permettant de connaître la localisation précise de la tête fœetale et des instruments à l'intérieur du bassin maternel.

Ce type de simulateur a fait l'objet d'une publication [102].

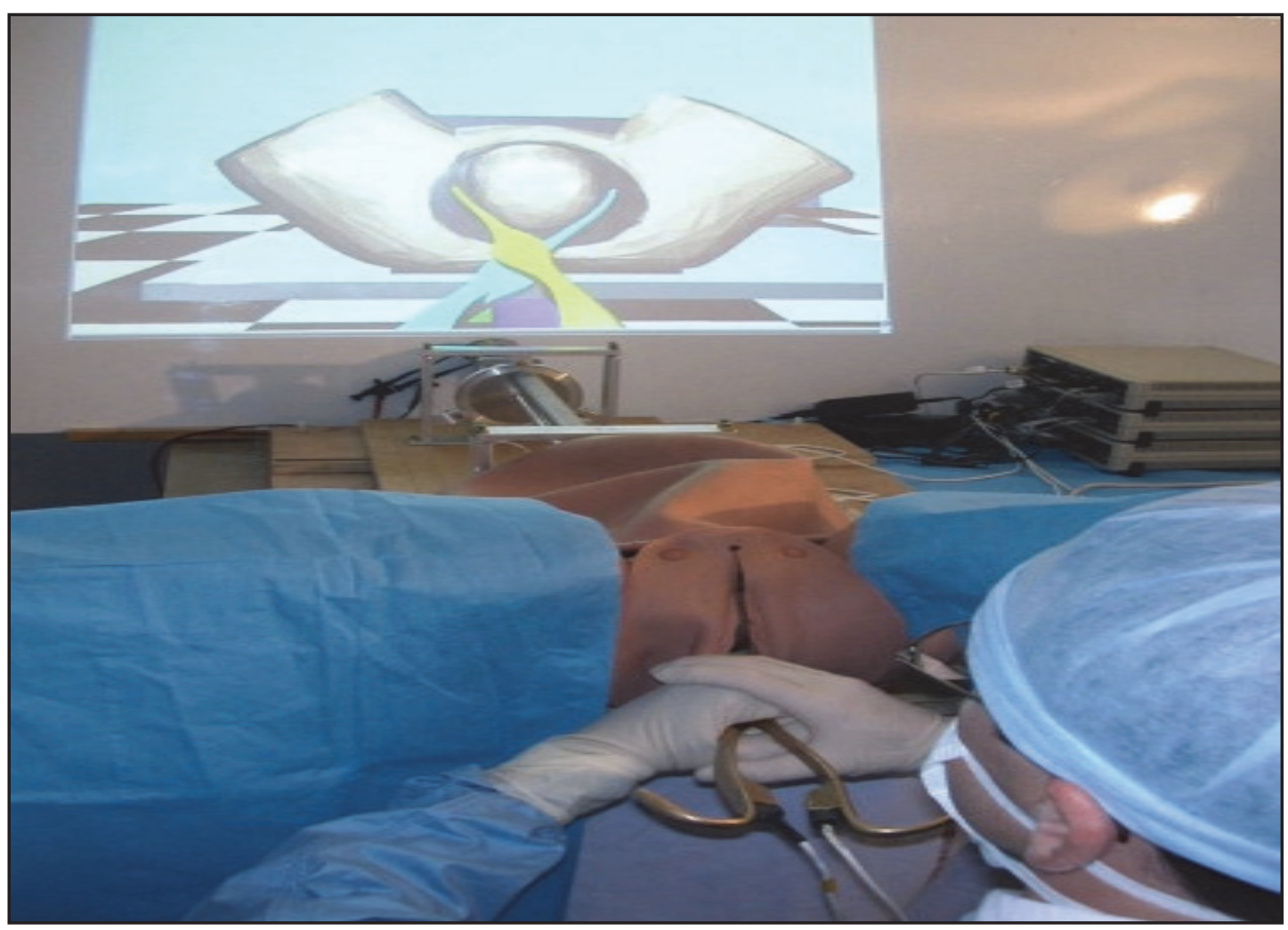

Figure 30 : The BirthSIM Simulator. 
Les simulateurs comme outil d'enseignement diagnostique et thérapeutique peuvent permettre une éducation par la répétition. La répétition des exercices et l'évaluation sont deux étapes majeures du modèle d'apprentissage [111].

Ainsi, la possibilité de réaliser en toute confiance certains gestes techniques par une répétition à volonté permet d'augmenter le niveau de performance de l'étudiant en réduisant le vide entre la courbe d'apprentissage et celle de l'efficacité sur le terrain. Ces notions ont clairement été décrites [111] [Figure 31].

Ainsi les simulateurs obstétricaux aux EI permettraient la répétition d'un exercice donné et participeraient à l'apprentissage du geste d'EI.

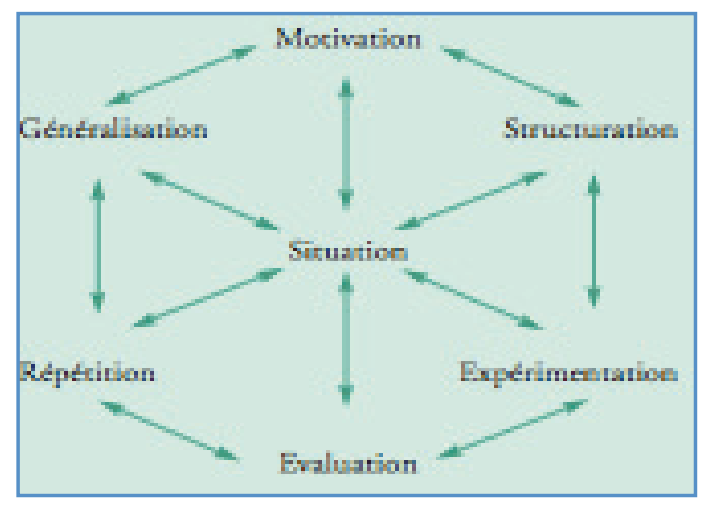

Figure 31 : Description du modèle d'apprentissage : les étapes du modèle de Butler.

L'apprentissage par répétition est également permis par les cours de DES, les diplômes interuniversitaires, les nouvelles technologies de l'information et de la communication, les sites dédiés à la vidéo.

\section{Formation au «savoir décisionnel»}

La mise en situation d'un accouchement par EI doit être réaliste ou faire intervenir une simulation, idéalement « haute fidélité » c'est-à-dire avec un degré élevé de réalisme avec en particulier l'intervention d'acteurs mimant les participants (patiente, sage-femme, anesthésiste réanimateur, pédiatre, équipe paramédicale).

La toute nouvelle génération de simulateur intègre une technicité qui permet une interactivité. Un modèle proposé est le modèle Laerdal, SimMom ${ }^{\circledR}$. Lorsqu'une équipe s'entraine sur ce simulateur, la qualité de communication peut être évaluée en même temps que les gestes 
techniques de prise en charge médicale. Cet outil va coupler les fonctionnalités du bassin osseux et $\mathrm{du}$ fœetus. Une interface informatique permet de simuler des variations des paramètres vitaux comme la tension artérielle maternelle et l'enregistrement d'un rythme cardiaque fotal [112] [Figure 32].

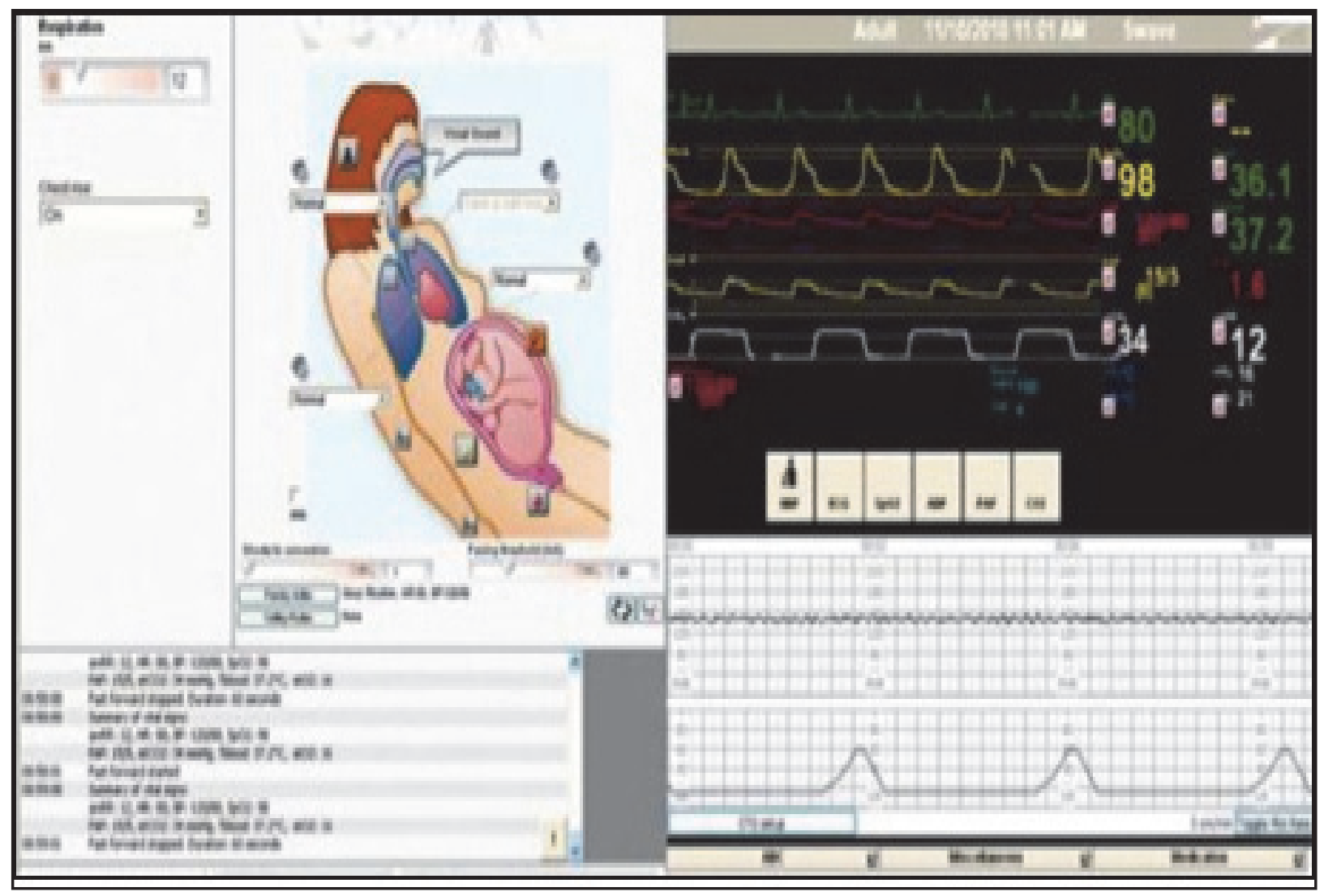

Figure 32 : Laerdal : console de paramétrage SimMom ${ }^{\circledR}$.

L'objectif d'une formation optimale est de réaliser le couplage entre l'enseignement théorique et la pratique en situation.

Les solutions concrètes proposées par ce simulateur sont nombreuses :

- acquisition de tâches spécifiques par la répétition ;

- actualiser ses connaissances et compétences en situation ;

- mise en situation « réelle » avec mannequin qui parle, respire, qui a un pouls et une pression artérielle mesurable, dans un environnement de travail comme une salle d'accouchement ; 
- une vitre sans tain sépare les apprenants des instructeurs et autres étudiants qui observent la scène. La séance de débriefing qui suit et le revisionnage de la simulation enregistrée permettent en quelques minutes d'en extraire les points clés et les erreurs.

Ainsi, la prise en charge par le groupe évalué peut être observée, les gestes techniques sont critiqués mais également les gestes non techniques comme la relation avec le patient, la coordination et la cohésion entre les différents membres du groupe.

D’une façon plus générale, la prise de décision et la prise en charge globale du patient sont facilitées par cet entraînement en équipe. Les scénarii proposés seront progressifs, en partant de situations simples (gestes fréquents) jusqu'à des situations plus complexes et rares. On pourra alors observer si les gestes et les thérapeutiques sont appropriés, mis en œuvre dans des délais conformes et en parfaite ergonomie avec les équipes.

Les gestes d'urgence ont principalement un apprentissage théorique. Ce constat pousse à réfléchir sur une approche pédagogique optimale pour obtenir la meilleure performance dans des situations pathologiques rencontrées ultérieurement. C'est en situation « réelle » que ces gestes s'acquièrent le mieux. En situation d'urgence, les gestes techniques doivent être fiables et parfaitement réalisés. Il n'y a pas de place pour l'improvisation. Un simulateur très proche du réel permet un entraînement et la répétition à volonté de ces situations, sans les questions éthiques soulevées par les situations avec un véritable patient.

Les choix d'utilisation de la simulation sont multiples. La formation d'une équipe est fondamentale et peut se réaliser sur simulateur. Des scénarii peuvent intégrer des événements imprévus qui ont tendance à augmenter les risques et les erreurs de prise en charge.

D’autres outils participes à la formation au savoir décisionnel :

- le compagnonnage (maternités des CHU et maternités accueillant des internes).

- les revues de morbidité des services : l'EBCOG recommande des réunions régulières avec études de dossiers et revue de morbidité et de mortalité. 


\section{4) Comment évaluer cette formation?}

Compte tenu de l'importance de la formation, l'évaluation de celle-ci est indispensable.

Cette évaluation concerne à la fois les apprenants et les formateurs.

Une évaluation de la procédure de formation au moins annuelle est prônée par le CEGO (EBCOG Training Assessment Edit 18/03/ 2008) [15].

Un outil d'évaluation valide des EI par ST pourra permettre l'évaluation de cette formation.

Les facteurs qui influencent le niveau de compétence d'un étudiant dans une procédure donnée sont nombreux. Quand on interroge des étudiants et formateurs à ce sujet, deux thèmes sont mis en avant :

- le niveau de compétence de l'étudiant dans une procédure clinique

- le niveau d'indépendance accordé et acceptable par l'étudiant

Ces facteurs sont régis par les concepts d'expérience, des connaissances, de la pédagogie ,de l'autoévaluation, de l'activité clinique, de l'environnement de travail, et notions plus abstraite tel que le ressenti [113].

Certains modèles d'évaluation des connaissances théoriques sur simulateur existent. Dans une étude récente, des étudiants ont participé à un exercice sur simulateur haute-fidélité d'un accouchement assisté par une EI, suivi d'une évaluation écrite dans laquelle devait être documenté le déroulement de l'accouchement. 14 items jugés essentiels par la SOGC, concernant la documentation appropriée d'un accouchement vaginal par EI assisté devaient y figurer. Cependant l'amélioration des compétences théoriques de l'étudiant n'a pu être mise en évidence au vue de l'absence de répétition de cet exercice [114].

Les exercices sur simulateurs peuvent être utilisés en tant que modalité d'évaluation. 


\section{Validation des connaissances factuelles et conceptuelles}

Par l'obtention du DES de gynécologie et obstétrique ainsi que du diplôme interuniversitaire (DIU) de mécanique et technique obstétricale.

Ce DIU permet un enseignement diversifié de ce qui se fait dans les salles de naissances françaises en prenant en compte l'expérience de chacun dans le respect de la mécanique obstétricale et du bien être maternel et fœtal. Ce DIU utilise différents supports d'apprentissage: livres, diapositives, films, travaux pratiques sur mannequins et vidéotransmission.

La formation à l'EI doit faire l'objet d'une validation initiale lors de l'obtention du DES : une présentation systématique par les internes d'un carnet de bord comprenant le nombre d'EI, le type d'instrument utilisé, le taux d'échec et le degré d'autonomie est souhaitable.

\section{$>$ Validation des connaissances procédurales et décisionnelles}

* En réalisant un certain nombre d'EI dans une mise en situation réelle.

En réalisant d'un certain nombre d'extractions instrumentales sur simulateur.

Une copie des comptes rendus manuscrits des EI permettant une documentation du déroulement de l'accouchement.

Glenn et al soulignent l'importance de cette documentation tant sur le plan médical que légal [114].

En complétant un cahier de stages, de type livret de l'interne de l'AGOF. Depuis 2003, sur le modèle du log-book créé par l'EBCOG [115].

La commission universitaire du CNGOF, en partenariat avec l'AGOF (Association des gynécologues obstétriciens en formation), a réalisé le livret de l'interne [105].

Ce dernier est un véritable outil de suivi et d'évaluation sur cinq ans du gynécologue obstétricien en formation.

Il propose notamment la mise en place d'un tutorat formalisé à chaque stage. On y retrouve les textes officiels régissant l'internat de spécialité et le programme précis du DES de GO, en 11 modules.

Les modalités d'évaluation des internes en formation y sont décrites. Enfin, une liste des 
procédures pratiques à acquérir avec un nombre optimum de gestes à réaliser y est proposée [Tableau 12]. Le livret évalue chaque semestre.

Il contient tout d'abord un document fixant les objectifs de début stage (théorique, pratique et scientifique) à remplir conjointement par le responsable du stage et l'interne. Puis, quatre documents sont à remplir en fin de stage (attitudes, procédures chirurgicales, procédures obstétricales et échographie) sous forme d'auto- et d'hétéro évaluation.

Ainsi, chaque semestre est sanctionné sous la forme d'un bilan type «avant-après ».

Plus qu'un énième moyen d'évaluation, il s'agit, en fait, d'un outil pédagogique permettant de suivre sa progression et de se fixer régulièrement des objectifs. Ce livret sert également à faire connaître au tuteur en début de stage les compétences acquises et ainsi à négocier avec lui l'apprentissage de nouveaux gestes.

En utilisant le Log Book européen (EBCOG Training assessment Edit 18/03/2008).

Elle permet une autoévaluation des apprenants et évaluation .au moins annuelle par les tuteurs $[15,115]$.

* En utilisant une fiche structurée d'évaluation des compétences pour l'EI.

(RCOG 18/03/2008)[26]. Aucune donnée n'est disponible dans la littérature concernant la validation d'un outil d'évaluation dans les extractions par ST.

* En éditant un rapport confidentiel pour chaque praticien.

Une mesure de ce type a été décrite par Lane à Liverpool en 2007 (NP4) [116]. La formation continue peut reposer sur des données remises chaque année de manière confidentielle à chaque praticien : en particulier en ce qui concerne le taux d'EI et le taux d'échec de pose pour chaque instrument [85]. 
Tableau 12 : Nature et nombre de gestes à réaliser pendant l'internat de gynécologie obstétrique.

\begin{tabular}{|l|l|}
\hline Acte & Nombre \\
\hline Consultation & \\
\hline Consultation programmée & 50 \\
Echographie & 300 \\
\hline Obstétrique & \\
\hline Accouchement (dont siège et grossesse multiple) & 50 \\
Extraction instrumentale (Forceps, Vacuum...) & \\
Césarienne & 40 \\
Amniocentèse & 40 \\
& 10 \\
& \\
\hline Gynécologie & 15 \\
\hline Induction de l'ovulation, Ponction FIV & 50 \\
Colposcopie & 10 \\
Conisation & 35 \\
Hystéroscopie diagnostique et chirurgicale & 20 \\
Curetage/IVG & 60 \\
Coelioscopie diagnostique et chirurgicale & 10 \\
IUE & 20 \\
Hystérectomie, Prolapsus & 20 \\
Chirurgie mammaire (tumorectomie, curage, mastectomie) & \\
\hline
\end{tabular}

\section{5) Améliorer la formation en gynécologie obstétrique grâce à la simulation}

La formation en GO a fait l'objet de diverses discussions.

Dans un communiqué l'AGOF proposait une réflexion afin de discuter de l'intérêt pédagogique de la simulation en GO, à la lumière du contexte démographique et de la mise en application de multiples réformes [117,118]. Le modèle de formation et d'enseignement actuel est fondé sur l'intégration des internes dans les équipes médicales. Tant théorique que pratique, l'apprentissage se fait au contact des plus anciens et le compagnonnage sous le contrôle d'un senior permet une mise en responsabilité progressive en chirurgie et en obstétrique. Si le compagnonnage a pendant des années fait la preuve de sa très grande efficacité dans la formation clinique, l'acquisition de la technique, son efficience requiert un volume élevé de gestes obstétricaux et de procédures chirurgicales. 
Cet enseignement, en salle de naissance ou au bloc, nécessite du temps et ne peut être garanti que si le nombre d'étudiants reste raisonnable. Pour rappel la création du DES de GO date de 1999, en quelques années, la formation des futurs gynécologues obstétriciens français a été profondément remaniée [119]

En janvier 2012, un rapport de l'HAS faisait état de 10 propositions pour une amélioration de la qualité et la sécurité des soins en France, centré sur le développement de la simulation dans le domaine de la santé [120]. Le premier point de recommandation préconisait le développement et l'intégration d'une formation par les méthodes de simulation dans tous les programmes d'enseignement des professionnels de santé à toutes les étapes de leur cursus (initial et continu).

Leur intégration tout le long du troisième cycle de l'internat de GO peut être complémentaire de la formation actuelle tel que le préconise ce rapport de l'HAS [120]. En Amérique du Nord, la simulation est un outil pédagogique à part entière, elle est utilisée de manière routinière dans l'enseignement initial des professions médicales. En Europe, la simulation est un outil pédagogique bien implanté, mais les inégalités restent importantes. La question de coût est une limite majeure à la généralisation de ces centres.

En France et en GO, la simulation et la recherche sur ce thème sont une activité émergente qui peut déjà s'appuyer sur quelques centres comme le Centre international de chirurgie endoscopique (CICE). A l'échelle locale, la faculté de médecine de Nice a mis en place un programme pédagogique qui intègre la simulation dans la formation des internes en chirurgie, auquel les internes de GO participent [121].

En obstétriques diverses situations peuvent être simulées: la dystocie des épaules, l'hémorragie de la délivrance, les contextes de pré éclampsies ou d'éclampsie, l'accouchement par le siège, les accouchements assistés par EI ou les procédures de ponctions (amniocentèse, ponction fotale)[122]. Dans le domaine du diagnostique anténatal les simulateurs d'amniocentèse ont permis d'améliorer les pratiques en accélérant l'apprentissage et en facilitant l'évaluation des compétences [123]. 
La simulation en obstétrique a fait l'objet de diverses publications classées en 3 catégories :

- description de modèles de simulateurs ou d'exercice sur simulateur

- description de modèles de simulateurs ou d'exercice sur simulateur et évaluation des compétences de l'étudiant avant et après simulation

- description d'un ou plusieurs modèle de simulateur ou d'exercice sur simulateur en l'intégrant dans un programme multidisciplinaire ou dans un programme de formation en équipe.

Nous insistons sur l'importance de l'apprentissage par simulateurs, indispensable pour le gynécologue obstétricien en formation. 


\section{VI - CONCLUSION}

La formation aux extractions par spatules de Thierry pendant l'internat de GO n'expose pas le fotus à un risque supplémentaire. L'approche pédagogique de notre étude ne permet pas de donner une valeur seuil unique du nombre d'extractions par ST nécessaires pour juger de la compétence de l'intégralité des internes, toutefois une progression significative de ces extractions, durant la période de suivi a été observée. Ainsi, des modèles d'évaluations des compétences des internes appliqués à l'obstétrique devront faire l'objet d'études complémentaires. Un nombre de 20 procédures dans le cadre de l'apprentissage des extractions instrumentales par spatules de Thierry est une valeur seuil à partir de laquelle les internes s'estiment subjectivement capables de pratiquer sous la supervision de médecins séniors. Il s'agit également d'une valeur à partir de laquelle les médecins séniors estiment que les internes sont suffisamment compétents pour pratiquer ces extractions sous leur supervision.

Ce seuil de 20 procédures doit, à notre sens, être non seulement interprété sous un angle éducationnel mais également sous un angle clinique avant de permettre un apprentissage sur patiente et ce, afin de limiter des risques inutiles de morbi-mortalités materno-fœtales liés à une pratique trop précoce. Cela conforte l'idée qu'un plus grand nombre d'extractions par spatule est indispensable avant d'être compétent, et pourrait rejoindre les chiffres des recommandations européennes.

Les simulateurs obstétricaux sont un outil d'apprentissage qui semble devoir devenir indispensable puisqu'ils permettent une pratique objectivement évaluable, jusqu'à atteindre les seuils de pratique, en sécurité, des extractions instrumentales. Le cursus médical devrait comporter, notamment pour les extractions instrumentales, l'utilisation de courbes d'apprentissage sur simulateur au sein desquelles des seuils minimum doivent être déterminés avant qu'une exposition clinique ne soit autorisée. L'utilisation de simulateurs obstétricaux devrait ainsi pouvoir permettre la réalisation des premières phases d'apprentissage des internes, en évitant d'exposer les patientes à un sur-risque de morbidité. De nouveaux travaux sont nécessaires pour étudier les possibilités offertes par les simulateurs pour l'apprentissage des extractions instrumentales par spatules de Thierry. 


\section{VII - ANNEXES}

\section{A - ANNEXE 1}

\section{Fiche d'évaluation à remplir par l'obstétricien de garde}

\begin{tabular}{|c|c|c|}
\hline & $\begin{array}{l}\text { échelle } 1 \text { à } 5 \\
\text { (5=excellent) }\end{array}$ & Non évalué \\
\hline \multicolumn{3}{|l|}{ Préparation } \\
\hline Analgésie & $1----2----3----4----5$ & \\
\hline Dilatation complète & $1----2----3----4----5$ & \\
\hline Asepsie & $1----2----3----4----5$ & \\
\hline Evaluation du niveau d'engagement & $1----2----3----4----5$ & \\
\hline Evaluation du type de présentation & $1----2----3----4----5$ & \\
\hline SU évacuateur & $1----2----3----4----5$ & \\
\hline Information de la patiente & $1----2----3----4----5$ & \\
\hline \multicolumn{3}{|l|}{ Pose des spatules } \\
\hline Mouvement de pose 1ére spatule & $1----2----3----4----5$ & \\
\hline Mouvement de pose 2ème spatule & $1----2----3----4----5$ & \\
\hline Protection des tissus mous & $1----2----3----4----5$ & \\
\hline \multicolumn{3}{|l|}{ Extraction } \\
\hline Coordination avec EE et patiente & $1----2----3----4----5$ & \\
\hline Axe de traction correct & $1----2----3----4----5$ & \\
\hline Propulsion & $1----2----3----4----5$ & \\
\hline Mouvements d' « asynclitisme » & $1----2----3----4----5$ & \\
\hline Geste de dégagement de la tête & 1----2----3----4----5 & \\
\hline Contrôle du périnée post & $1----2----3----4----5$ & \\
\hline Geste du dégagement du corps & $1----2----3----4----5$ & \\
\hline Retrait des spatules & $1----2----3----4----5$ & \\
\hline $\begin{array}{l}\text { Episiotomie (moment-taille- } \\
\text { localisation) }\end{array}$ & $1----2----3----4----5$ & \\
\hline $\begin{array}{c}\text { Délivrance du placenta et des } \\
\text { membranes }\end{array}$ & $1----2----3----4----5$ & \\
\hline Vérification de la tonicité utérine & $1----2----3----4----5$ & \\
\hline Evaluation des lésions périnéales & $1----2----3----4----5$ & \\
\hline Estimation des pertes sanguines & $1----2----3----4----5$ & \\
\hline $\begin{array}{c}\text { Interaction avec le reste du personnel } \\
\text { soignant }\end{array}$ & $1----2----3----4----5$ & \\
\hline Rédaction du compte-rendu & $1----2----3----4----5$ & \\
\hline \multicolumn{3}{|c|}{ Je considère qu'à ce niveau de difficulté, l'interne est compétent pour : (cocher) } \\
\hline \multicolumn{3}{|l|}{ - observer/assister } \\
\hline \multicolumn{3}{|c|}{ - réaliser la procédure avec une supervision directe } \\
\hline \multicolumn{2}{|c|}{ - réaliser la procédure avec une supervision indirecte } & \\
\hline \multicolumn{2}{|l|}{ - réaliser la procédure sans supervision } & \\
\hline \multicolumn{2}{|l|}{ - enseigner la procédure } & \\
\hline - ne se prononce pas & & \\
\hline
\end{tabular}

Commentaires? Retrait des spatules au petit couronnement? O / N 


\section{B - ANNEXE 2}

\section{Fiche d'évaluation à remplir par l'interne de Garde}

\begin{tabular}{|c|c|c|}
\hline Interaction Senior/Interne (cocher) & OUI & NON \\
\hline Senior présent & & \\
\hline Aide verbale par le senior & & \\
\hline Aide manuelle par le senior & & \\
\hline
\end{tabular}

Si le médecin senior a dû vous aider, la modification du geste a eu lieu au niveau de :

\begin{tabular}{|l|l|l|l|}
\hline Pose des spatules & & Épisiotomie & \\
\hline Traction/Propulsion & & Protection du périnée & \\
\hline Retrait des spatules & & Dégagement & \\
\hline Autre : (préciser) & \multicolumn{3}{|l|}{} \\
& & \\
\hline
\end{tabular}

Entourer la réponse voulue

1. Ce cas vous a permis de progresser dans l'acquisition du geste d'extraction par spatules.
a. Tout à fait d'accord
b. D'accord
c. Neutre
d. Pas d'accord
e. Pas du tout d'accord
f. Ne se prononce pas

2. Comment évaluez vous la difficulté de cette extraction?
$a$. Très facile
b. Facile
c. Moyen
d. Difficile
e. Très difficile
f. Ne se prononce pas

3. Quel est votre niveau de confiance pour les extractions par spatules?
a. Pas du tout en confiance
b. Nécessité d'une supervision rapprochée
c. Nécessité d'une supervision minimum
d. Peut réaliser des extractions indépendamment avec un soutient en cas de problème
e. Peut réaliser des extractions indépendamment
f. Ne se prononce pas

Commentaires? Lésions néonatales? $\mathrm{O} / \mathrm{N} \quad$ Retrait des spatules au petit couronnement? 


\section{C - ANNEXE 3}

Grille d'évaluation des accouchements instrumentaux du RCOG

\section{OPERATIVE VAGINAL DELIVERY}

\begin{tabular}{|c|c|c|}
\hline Trahee Name: & Sol. Ye & Dase: \\
\hline Assessor Name & Poat & \\
\hline \multicolumn{3}{|c|}{$\begin{array}{l}\text { Clinical details of complexity/ } \\
\text { difticulty of case }\end{array}$} \\
\hline
\end{tabular}

\begin{tabular}{|c|c|c|}
\hline & $\begin{array}{l}\text { Rerifarmited } \\
\text { Inslependenthy }\end{array}$ & $\begin{array}{l}\text { Needs } \\
\text { help }\end{array}$ \\
\hline & \multicolumn{2}{|c|}{ PLEASETICK RELENANT BOX } \\
\hline \multicolumn{3}{|l|}{ Itema under observation: operning } \\
\hline \multicolumn{3}{|l|}{ Eneure parient and accomparying partier understand procedure } \\
\hline \multicolumn{3}{|l|}{ Appropriate preoperative preparation adequabe anilgeia, bladder empty } \\
\hline \multicolumn{3}{|l|}{ 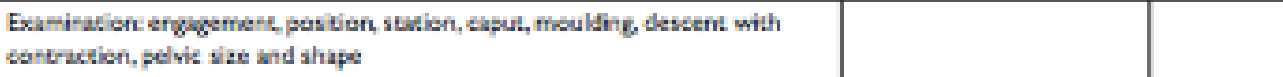 } \\
\hline \multicolumn{3}{|l|}{ Decision making chaice of instr-memt } \\
\hline \multicolumn{3}{|l|}{ Correct asse-ably and checking of equigmene } \\
\hline \multicolumn{3}{|l|}{ Correct applicatian of instrument } \\
\hline \multicolumn{3}{|l|}{$\begin{array}{l}\text { Appropriate elinection force and timing of put. f-sures head dexcends with } \\
\text { trattios }\end{array}$} \\
\hline \multicolumn{3}{|l|}{ Appropriate atberation of traction with delivery of head } \\
\hline \multicolumn{3}{|l|}{ Protects perineum and aspess need for episiocomy } \\
\hline \multicolumn{3}{|l|}{ Chocks Sor terd Cerrect delmery of thaulders and body } \\
\hline \multicolumn{3}{|l|}{ Delivery of plscenta and membranes } \\
\hline \multicolumn{3}{|l|}{ Chocks bor uterite laxicy and waginal trauma } \\
\hline \multicolumn{3}{|l|}{ Estimated flood toss and manoges blood loss } \\
\hline \multicolumn{3}{|l|}{ Approprlate wse of tes- } \\
\hline \multicolumn{3}{|l|}{ Awareness of maternal and fetal melbeing throughouta } \\
\hline Comments: & & \\
\hline
\end{tabular}

Examples of minimum levels of complexity for each stage of training

Basic Training

Intermediate Training

Advanced
Uncomplicated. Non rotational

Rotztional ventouse

Rotational forceps/ventouse in thentre

Both sides of this form to be completed and signed 
GENERIC TECHNICAL SKILLS ASSESSMENT Assessor, please ring the candidate's performance for each of the following factorst

\begin{tabular}{|c|c|c|c|}
\hline Respect har clasue & 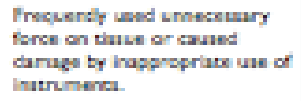 & $\begin{array}{l}\text { Cardul handing of diule bur } \\
\text { socationaly cautet inadvertert } \\
\text { damage. }\end{array}$ & 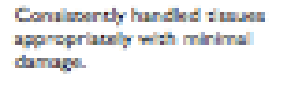 \\
\hline $\begin{array}{l}\text { Time, motion and flow } \\
\text { of ogeration and } \\
\text { forward planning }\end{array}$ & 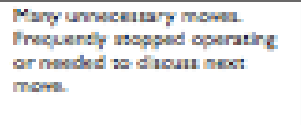 & 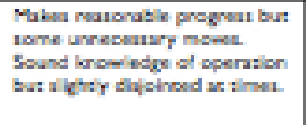 & 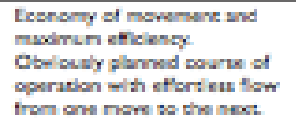 \\
\hline $\begin{array}{l}\text { Kromiedge and handing } \\
\text { of kstrumbents }\end{array}$ & 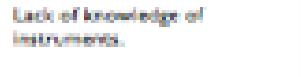 & 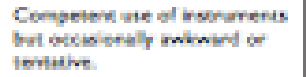 & 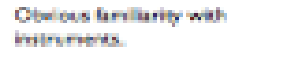 \\
\hline $\begin{array}{l}\text { Sucurine and buotting } \\
\text { skills as appropriate har } \\
\text { Che procedure }\end{array}$ & 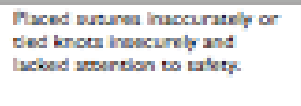 & 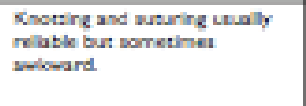 & 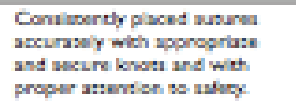 \\
\hline $\begin{array}{l}\text { Technical uas of } \\
\text { asaivtanta } \\
\text { Relations with patient } \\
\text { and the aurgical team }\end{array}$ & 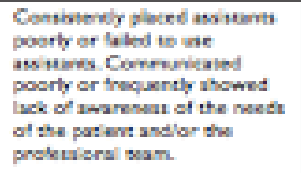 & 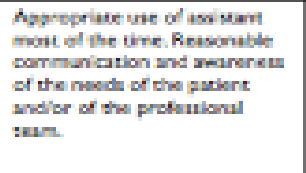 & 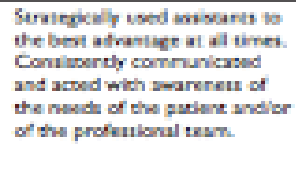 \\
\hline Insigent/atsicute & $\begin{array}{l}\text { Poor woblertionding of areis of } \\
\text { neakines. }\end{array}$ & $\begin{array}{l}\text { Some nolerionding of areis of } \\
\text { wewhens. }\end{array}$ & 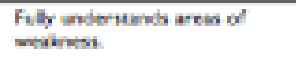 \\
\hline $\begin{array}{l}\text { Desommentation of } \\
\text { procedures }\end{array}$ & $\begin{array}{l}\text { Uimbed decumertablen, poorly } \\
\text { whimen. }\end{array}$ & 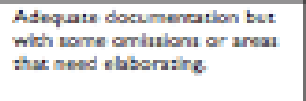 & 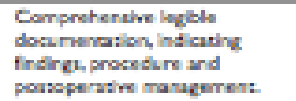 \\
\hline
\end{tabular}

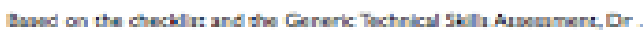

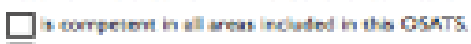

$\square$ in working sowartt competence.

\begin{tabular}{|c|c|}
\hline Noth folver leb wit & 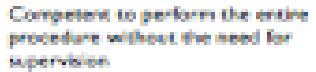 \\
\hline Done & Daie \\
\hline syend pravend & Sgend \\
\hline saned (proviel) & 5 gend \\
\hline
\end{tabular}

Delete whers applicable, and tuse and lign the rekrant box 


\section{D - ANNEXE 4}

Compte rendu type des extractions instrumentales par spatules de Thierry

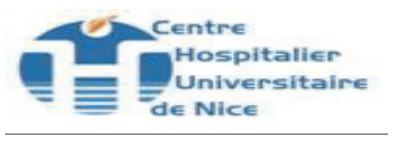

\section{Service de Gynécologie obstétrique \\ Reproduction et Médecine Foetale}

Centre « Femme-Mère-Enfant »

Professeur A. BONGAIN - Chef de

service

COMPTE RENDU DES EXTRACTIONS PAR SPATULES DE THIERRY

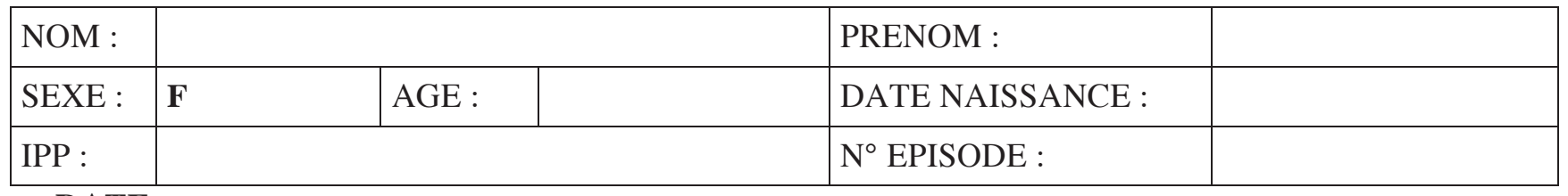

DATE :

Heure début d'intervention :

Heure fin d'intervention :

Résumé de la grossesse :

\begin{tabular}{|l|l|l|}
\hline \multicolumn{1}{|c|}{ Gynécologue - Obstétricien } & Anesthésiste & Sage femme \\
\hline $\begin{array}{l}\text { Obstétricien de garde : } \\
\text { Interne : }\end{array}$ & & \\
\hline
\end{tabular}

INDICATION :

INTERVENTION : EXTRACTION FOETALE PAR SPATULES DE THIERRY

Sous : APD/AL/Rachianesthésie/AG/Bloc du nerf honteux interne

Installation de la patiente

- En position gynécologique et désinfection vulvo-vaginale

- Sondage vésical évacuateur.

Toucher Vaginal : présentation céphalique au Détroit supérieur/moyen/inférieur

Variété non évaluable/OIGA/OIGP/OIDA/OIDP/OP/OS

Bosse séro-sanguine : oui/non; assynclitisme : oui/non

Pose aisée/difficile/moyennement/facile des Spatules de Thierry

- Retrait des spatules au petit couronnement : oui/non

- Extraction en OP/OS à .... H .... d'un enfant de sexe féminin/masculin pesant .......g,

apgar ......./...

Délivrance dirigée/artificielle/naturelle complète/incomplète selon le Mode Baudelocque/Ducan

Réfection de l'épisiotomie isolé ou non

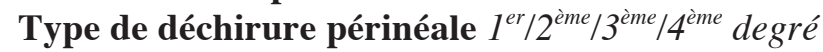

- Plan vaginal suturé par...

- Plan musculaire suturé par ...

- Plan cutané suturé par ...

Toucher Vaginal et Toucher Rectal : absence/présence de lésion et de point transfixiant ; retrait des compresses.

Pertes sanguines totales : ...

En fin de geste : saignements normaux, globe tonique

Soins post-opératoires :

Remarques :

Signature du Médecin Intervenant : 


\section{E - ANNEXE 5}

\section{Définitions des qualités d'un instrument de mesure}

\begin{tabular}{|c|c|}
\hline$\frac{\text { Validité de }}{\text { contenu }}$ & $\begin{array}{l}\text { Des experts évaluent dans quelle mesure les items composant l'échelle } \\
\text { sont pertinents et constituent un échantillon représentatif de l'univers de } \\
\text { tous les items possibles pouvant décrire le phénomène mesuré. }\end{array}$ \\
\hline$\frac{\text { Validité contre }}{\text { critère }}$ & $\begin{array}{l}\text { Le phénomène mesuré est évalué à la fois par l'échelle étudiée et un } \\
\text { critère extérieur à celle-ci pris comme référence (Gold Standard). Un } \\
\text { nombre suffisant de sujets étant évalué de manière indépendante par les } \\
\text { deux instruments, on mesure l'intensité du lien statistique existant entre } \\
\text { les deux évaluations. }\end{array}$ \\
\hline $\begin{array}{l}\text { Validité de } \\
\text { construit }\end{array}$ & $\begin{array}{l}\text { La conception théorique que nous avons du phénomène mesuré par } \\
\text { l'échelle permet de faire un certain nombre d'hypothèses. Celles-ci } \\
\text { seront ensuite testées expérimentalement. La validité du construit } \\
\text { s'affirme et croît donc peu à peu, au fur et à mesure que } \\
\text { des expériences successives viennent confirmer les hypothèses } \\
\text { préalables. Mais le processus de validation n'est jamais terminé, d'autres } \\
\text { hypothèses devront être formulées et vérifiées. C'est ainsi que les très } \\
\text { bonnes échelles ont une validité du construit résultant de nombreuses } \\
\text { expérimentations }\end{array}$ \\
\hline$\overline{\text { Fidélité }}$ & $\begin{array}{l}\text { On considère qu'un instrument de mesure est fidèle si le résultat qu'il } \\
\text { produit est reproductible. En d'autres termes, si on applique le même } \\
\text { instrument (ou des versions comparables de cet instrument) à une même } \\
\text { personne plusieurs fois de suite, cette personne doit être caractérisée par } \\
\text { le même score . La fidélité peut être évaluée dans diverses situations } \\
\text { expérimentales. } \\
\text { - La fidélité inter juges : chaque personnes est alors coté au même } \\
\text { moment par deux (ou plus) juges différents, de manière indépendante } \\
\text { - La fidélité test-retest : c'est une situation semblable à la fidélité intra } \\
\text { juge, mais cette fois la personne s'autoévalue lui même, il est donc son } \\
\text { propre juge. } \\
\text { Quelle que soit la situation, la fidélité est d'autant plus élevée que les } \\
\text { scores obtenus par chaque sujet sont proches. }\end{array}$ \\
\hline $\begin{array}{l}\text { Sensibilité au } \\
\text { changement }\end{array}$ & $\begin{array}{l}\text { Un instrument est dit sensible au changement s'il est capable de mesurer } \\
\text { avec précision les variations en plus ou en moins, du phénomène mesuré }\end{array}$ \\
\hline$\frac{\text { Cohérence }}{\text { interne }}$ & $\begin{array}{l}\text { Le coefficient alpha de Cronbach évalue la cohérence interne d'un } \\
\text { ensemble d'items, échelle ou sous échelle, correspondant à une } \\
\text { dimension clinique unique ; c'est-à-dire la force des inter corrélations } \\
\text { entre items. Plus les items sont liés entre eux, plus la valeur d'alpha est } \\
\text { grande. }\end{array}$ \\
\hline
\end{tabular}




\section{F - PUBLICATION SOUMISE}

Article soumis à la revue « The Journal of Obstetrics and Gynaecology Research »

Instrumental extractions using Thierry's spatulas:

evaluation of the learning curve

\section{Authors :}

El Haloui Ouidade ${ }^{1, *}$ MD.

Delotte Jérôme ${ }^{1} \mathrm{MD}, \mathrm{PhD}$

Boukaïdi Samir ${ }^{1}$ MD

Bongain André ${ }^{1} \mathrm{MD}, \mathrm{PhD}$

Boucoiran Isabelle ${ }^{1,2} \mathrm{MD}, \mathrm{MSc}$

\section{Affiliations :}

1- Department of Obstetrics, Gynecology, Reproduction and Fetal Medicine. University Hospital Center of Nice (Archet II). University of Nice-Antipolis. 151, route St Antoine de Ginestière. 06202 Nice (France).

2- BC Women's Hospital and Health Centre B327-4500 Oak Street, Box 42 Vancouver, BC V6H 3N1 (Canada).

\section{* Corresponding author:}

El Haloui Ouidade, MD

Tel : + $0033(0) 492036108$

Fax : + 0033 (0)4 92035949

E-mail : ouidade@hotmail.com

Keywords: Fetal extraction; Thierry's spatulas; Resident; Learning curve; Childbirth. 


\begin{abstract}
Objective: To determine the learning curve of fetal extractions with Thierry's spatulas.

Materials and methods: Single-center prospective study following the progress of six residents in obstetrics and gynecology in learning fetal extractions by Thierry's spatulas. The instrumental extractions procedures performed by the residents were evaluated by the senior obstetrician on call according to a validated scoring rubric. In parallel, the residents would fill out a questionnaire on their own perception of their abilities at the time of the procedure.
\end{abstract}

Results: The six residents participated in 160 extractions using Thierry's spatulas between November and May 2011. Sixty-three (39.6\%) were evaluated, i.e. an average number of $12+/-6$ extractions per resident. After validating respectively $0,1,2$ or 3 semesters, the mean global assessment scores (graded out of 5) obtained by the residents were 3.54, 3.18, 4.43 and 4.61. The overall average score was significantly higher when the resident participated in more than 20 extractions before the studied extraction $(4.0$ vs. 3.7 , p <0.001). After attending more than 20 extractions, no resident had obtained the maximum evaluation score of 5/5 nor was deemed capable of performing extractions without supervision.

Conclusion: Learning extractions with Thierry's spatulas, including an objective assessment by senior doctors and a self-assessment by the residents, is a constant process over the first six months of training. Specific training simulator should be developed to enable students to achieve a threshold number of extractions, ensuring the safety of the procedure in the birthing rooms. 


\section{Introduction}

Thierry's spatulas are a fetal extraction instrument developed in the 50s by Dr. Thierry (Fig. 1). This instrument is based on a mechanical principle of guidance and propulsion of the fetal body, each spoon acting as a lever, which rests on the maternal soft tissue and the fetal bone. Some of its advantages are well known by its users: fast implementation and protection of the fetus, even in cases of misdiagnosis of fetal presentation. However, the mechanism of action of this instrument is based on the propulsion, which can result in pressure on the female perineal tissue and increase the risk of severe perineal lesions $(1,2)$. The validation of the learning of medical students is an essential element to prevent complications related to such extractions. According to the European College of Obstetrics and Gynaecology, the learning curriculum of instrumental extractions by medical students must include at least 40 instrumental extractions, all categories included. However, the threshold value of the number of procedures to be carried out depending on the chosen instrumentation is not defined (3). If, in 1964, Lloyd had subjectively proposed a threshold of 20 spatula-assisted deliveries to be deemed competent, at present, there is no data on the learning curve of extractions using Thierry's spatulas (4).

The aim of our study was to determine the learning curve of this fetal extraction instrument and deduce if the threshold of 20 procedures was sufficient to achieve these extractions selfsufficiently.

\section{Materials and methods}

A single-center prospective study was conducted in the Department of Obstetrics and Gynecology, Reproduction and Fetal Medicine in Nice University Hospital. Between November 2010 and May 2012, the extractions performed with Thierry's spatulas by six residents in their early course (less than 2 years of practice) were evaluated by the senior doctors present at birth according to a grading system, built on a clinical audit model and based on the objectives of training of instrumental extractions as described by Dupuis (5), Sentilhes et al. (6) and the instrumental deliveries scoring rubric of the Royal College of Obstetricians and Gynecologists (7). The validity of its content was checked with three experts (each with over 10 years experience of births attended by spatulas) who confirmed the representativeness of the different items of the rubric. This allows to assign a score of 1 to 5 according to the quality of information given to the patient, the extraction gesture, the expulsion of the fetus, and the control of the perineum (Appendix 1). The total score was calculated as the sum of points awarded. A final item allowed to give an assessment of the 
overall level of competence of the resident for the completion of the extraction. In an independent manner, in an evaluation document inspired by the work of Jude et al. (8), the residents assessed the quality of their extractions and their self-confidence as to their ability to perform this procedure independently (Appendix 2).

The inclusion criteria were the use of Thierry's spatulas for extracting a singleton fetus, alive, more than 32 weeks of gestation, in cephalic presentation, regardless of the level of presentation in the pelvic excavation, by one resident of the studied group. The exclusion criteria were the absence of immediate filling out of one of the two scoring rubrics by the senior physician and/or the resident, and spatula-assisted caesarean section.

The decision to perform an instrumental extraction was only taken by the senior doctor on call, based on the recommendations of the French National College of Obstetricians and Gynecologists (CNGOF) (9). He validated the indication, the level of engagement of the fetal head and the variety of presentation. This study followed the recommendations of the 2000 Helsinki Declaration of the World Medical Association.

\section{Statistical Analysis}

Data were centralized and anonymized in a computer database. A descriptive analysis of the characteristics of the sample was performed, as well as a comparison of the characteristics of deliveries resulting in an assessment with the characteristics of other deliveries (Fisher's exact test or Mann-Whitney test). The impact of the residents' curriculum and of their level of experience (categorized according to the number of spatula-assisted deliveries performed before the evaluated delivery - more than 20 versus 20 or less) on the overall level of competence according to the senior doctor was explored by the same tests. A mixed linear model (MLM) with random effects allowed to study in a multivariate manner the impact of the curriculum of the resident on his overall assessment, considering the identity of the resident as a random effect factor and taking into account the difficulty of extraction. Similarly, we studied the impact on the overall score of having or not performed at least 20 spatula-assisted extractions before the evaluated extraction. All statistical tests were carried out under bilateral hypothesis at a significance level of 0.05 using SPSS 19.0.0 software (C) SPSS Inc., Chicago, IL 1989-2010). 


\section{Results}

All residents were women. Two were evaluated over a period of a year and a half, three over a period of 1 year and one over a period of 6 months. At the beginning of the study, 4 of 6 were first semester residents and the other two had already validated two semesters, both in obstetrics and gynecology. During the study period, these six residents participated in 160 vaginal deliveries with spatulas, 63 of which (39.6\%) were evaluated. Each resident achieved on average $12+/-6$ extractions and was valued 4.8 times per semester. The mothers, indications, presentations and levels of extractions, and newborns characteristics were not significantly different, whether an assessment was performed or not at the time of delivery. A senior doctor was present at $94 \%$ of extractions performed by a resident. Data for the assessment of the competence of the residents for each item in the scoring rubric are summarized in Table I. No resident was found capable of performing extractions without supervision during the monitoring period.

According to the residents, the senior doctor provided them with verbal assistance in $77 \%$ of cases and manual assistance in $65 \%$ of cases. This assistance concerned more than half of the stages of instrumental delivery in $23 \%$ of deliveries. The residents judged $21 \%$ of extractions as difficult or very difficult. In $91 \%$ of deliveries, the residents had a feeling of improved management of the spatula-assisted extraction gesture. In $84 \%$ of deliveries, the residents felt able to achieve extraction with minimal or close supervision, while in $14 \%$ of cases, they did not feel confident at all.

\section{Learning curve and evaluation threshold of 20 extractions}

The total evaluation scores of the residents, depending on their curriculum, are shown schematically in Figure 2. The average overall scores are 3.54, 3.18, 4.43 and 4.61 on 5 after respectively $0,1,2$ or 3 validated semesters. There is no significant impact of the difficulty experienced in the extraction on this relationship.

The residents who were considered able to perform spatula-assisted extractions with indirect supervision had on average validated 2.8 semesters against 2.1 for those who needed direct supervision and 0.9 semesters for those who could only attend or observe the procedure $(P=0.003$, Fig. 3). According to the MLM, the overall mean score was significantly higher when the resident participated in more than 20 extractions before the studied extraction ( $4 \mathrm{vs}$ $3.7, P<0.001$, Fig. 4). Having completed more than 20 extractions was statistically associated 
with being recognized by the senior physician as capable of performing an extraction under direct or indirect supervision ( $100 \%$ vs. $68 \%, P=0.031)$.

The confidence level of the residents tended to increase with their curriculum $(P=0.06$, Fig. 5). Residents who had performed more than 20 extractions felt more frequently confident at the time of delivery $(100 \%$ vs. $68 \%, P=0.034)$, while still wishing to benefit from a close supervision by a senior doctor. A feeling of progress in the management of the gesture was felt in all residents whether they had performed or not more than 20 extractions.

\section{Discussion}

Instrumental fetal extractions require a mastery of the technique due to the maternal-fetal complications they are likely to generate (10). If the choice of instrument is sometimes dictated by obstetric indications, it is often a matter of personal preference or of obstetric “obedience". Training in instrumental extractions during obstetrics and gynecology residency should aim to ensure the technical mastery of obstetrical instruments available to guarantee optimal maternal and fetal safety (11). Yet, to date, few studies have focused on the learning curve of each mode of instrumental extraction and the instrumental extractions threshold that should be reached before independent practice remains unknown. In a recent study on the learning curve of the vacuum extractor, a significant improvement of early-course residents was observed on ten procedures; however, the number of procedures to be performed before the student is "self-sufficient" could not be determined (12). According to a study on forceps extraction simulator, 31 applications of this instrument are necessary before the student masters the practice for occipito-pubic presentations (13). Thierry's spatulas are known to have a quick learning curve, though no objective data whatever is available on the subject (4, 14). Its current learning is mainly based on companionship at the patient's bedside. This teaching modality, that is essential in the phase of perfecting and of "responsibility awareness and effectiveness" of the resident, seems of limited value in the initial learning phase. In fact this teaching is often provided in an emergency and forensic context. In addition, the evaluation of the capacity of the resident to be sort of self-sufficient is essentially based on subjective criteria. Our work is the first to focus on the learning curve of spatula-assisted extractions using a systematic and validated rubric scoring. Assessments not immediately completed during the extraction were excluded to avoid skewing the validity of the analysis by a retrospective evaluation. The strict application of these analysis criteria associated with the constraints of on-call activity explains the number of scores excluded in our study criteria. However, the lack of statistically significant differences between the characteristics of 
deliveries that have been evaluated and those of excluded deliveries reflects the representativeness of the assessments studied.

The evolution curve of residents' assessment scores by the senior doctor on the basis of length of study can be described as three exponential phases surrounding a rapid and steady rise corresponding to the acquisition of practical knowledge. The first phase corresponds to the initial stages of assimilation of the technique in theory. The second phase, marked by a sharp rise, corresponds to a phase when theoretical knowledge coexists with improved practical skills. Finally, the third phase marks a new slower rise, the benefits of practical experience allowing an improvement in the extraction gesture, but could not reach a perfect score due to time-limited follow-up. This is typical of learning a particular technique (15). Our work also sought to assess the threshold of 20 extractions, previously suggested being sufficient to allow the practice of these extractions independently (4). Senior doctors have found, through the use of a systematic rubric scoring, that no resident was able to perform an extraction on his own after 20 procedures. Similarly, on their side and in an independent manner, no resident judged he was confident enough to achieve all extractions with Thierry's spatulas after participating in 20 extractions. It is to be noted that neither the senior doctors nor the residents were informed of the choice of the threshold of 20 extractions for our study. Our results show that a number of 20 extractions is necessary to obtain a significant improvement in assessment scores and allow the resident to be deemed competent while enjoying the effects of supervision by a senior doctor. This threshold of 20 procedures should, in our view, be interpreted not only as an educational perspective but also from a clinical angle before allowing learning on patients, in order to limit unnecessary risk of maternal-fetal morbidity and mortality associated with too early practice (16). This concept is not dependent on the country of learning nor on the type of extraction since Powell et al., in a study conducted in North American residents in obstetrics at the end of the course, had revealed that they considered the residents lacked 20 forceps extraction procedures to be competent (17). Thus, resident competence assessment models applied to obstetrics should be studied further. Obstetric simulators are a learning tool that seems to have become essential for it allows an objective assessment, reaching instrumental deliveries security practice thresholds (5). 


\section{Conclusion}

A number of 20 procedures for learning Thierry's spatulas fetal extractions is a threshold value at which the resident feels subjectively able to practice under the supervision of senior doctors. There is also a value from which the senior doctors think, objectively, that residents are competent enough to perform these extractions under their supervision. The medical curriculum should include, especially for instrumental deliveries, the use of learning curves in which the minimum thresholds must be determined before an independent or supervised practice is authorized in clinical practice. The use of obstetric simulators should enable the achievement by residents of the early stages of learning, avoiding exposing patients to an increased risk of morbidity. 


\section{References}

[1] Schaal JP, Riethmuller D, Berthet J. Manœuvres instrumentales et manuelles lors de l'accouchement. Rev Prat 1999 ; 49 : 139-45.

[2] Berthet J. Forceps : cur, quando, comodo (pourquoi, quand, comment). Encycl Med Chir, Elsevier, Paris, Obstétrique, 5-095-A-20, 2003, 8 p.

[3] http://www.cngof.asso.fr/D_TELE/eu_logb.pdf

[4] Lloyd FP, Geisler HE, Lushbaugh H. Unarticulated forceps in operative obstetrics.The spatulas of Thierry. Am J Obstet Gynecol 1964; 90 : 925-8.

[5] Dupuis O. Formation et apprentissage des extractions. J Gynecol Obstet Biol Reprod 2008 ; 37 : S288-96.

[6] Sentilhes L, Gillard P, Descamps P, Fournie A. Indications et prérequis à la réalisation d'une extraction instrumentale: quand, comment et où ? J Gynecol Obstet Biol Reprod 2008; 37: S188-201.

[7] http://www.rcog.org.uk/files/rcog-corp/uploaded-files/ED-CORE-OSATS-OP-VagDelivery_0.pdf

[8] Jude D, Gilbert G, Magrane D. Simulation training in the obstetrics and gynecology clerkship. American journal of obstetrics and gynecology 2006; 195: 1489-1492.

[9] CNGOF. Recommandations pour la pratique clinique : extractions instrumentals. J Gynecol Obstet Biol Reprod 2008; 37: S173-299.

[10] Mazouni C, Bretelle F, Collette E, Heckenroth H, Bonnier P, Gamerre M. Maternal and neonatal morbidity after first vaginal delivery using Thierry's spatulas. Aust N Z J Obstet Gynaecol $2005 ; 45$ : 405-9. 
[11] Dupuis O. Formation et apprentissage des extractions. J Gynecol Obstet Biol Reprod $2008 ; 37: 288-296$.

[12] Velemir L, Vendittelli F, Bonnefoy C, Accoceberry M, Savary D, Gallot D. Learning curve of vacuum extraction in residency: A preliminary study. J Gynecol Obstet Biol Reprod 2009; 38: 421-29.

[13] Dupuis O, Decullier E, Clerc J, Moreau R, Pham MT, Bin-Dorel S, Brun X, Berland M, Redarce T. Does forceps training on a birth simulator allow obstetricians to improve forceps blade placement? Eur J Obstet Gynecol Reprod Biol 2011; 159: 305-9.

[14] Thierry E. Bull Fed Soc Gynec Obst 1953; 5: 328.

[15] Chweitzer, A. (1931). VII. Etude expérimentale de la courbe d'apprentissage. L'année psychologique, 32(1), 164-196.

[16] Powell J, Gilo N, Foote M, Gil K, Lavin J.P. Vacuum and forceps training in residency: experience and self-reported competency. J Perinatol 2007 ; 27 : 343-346.

[17] Boucoiran I, Bafghi A, Delotte J, Valerio L, Bongain A. Risques néonataux et périnéaux liés à l'apprentissage des spatules. J Gynecol Obstet Biol Reprod 2010; 39: 224-230. 


\section{Figures}

Figure 1. Thierry's spatulas.

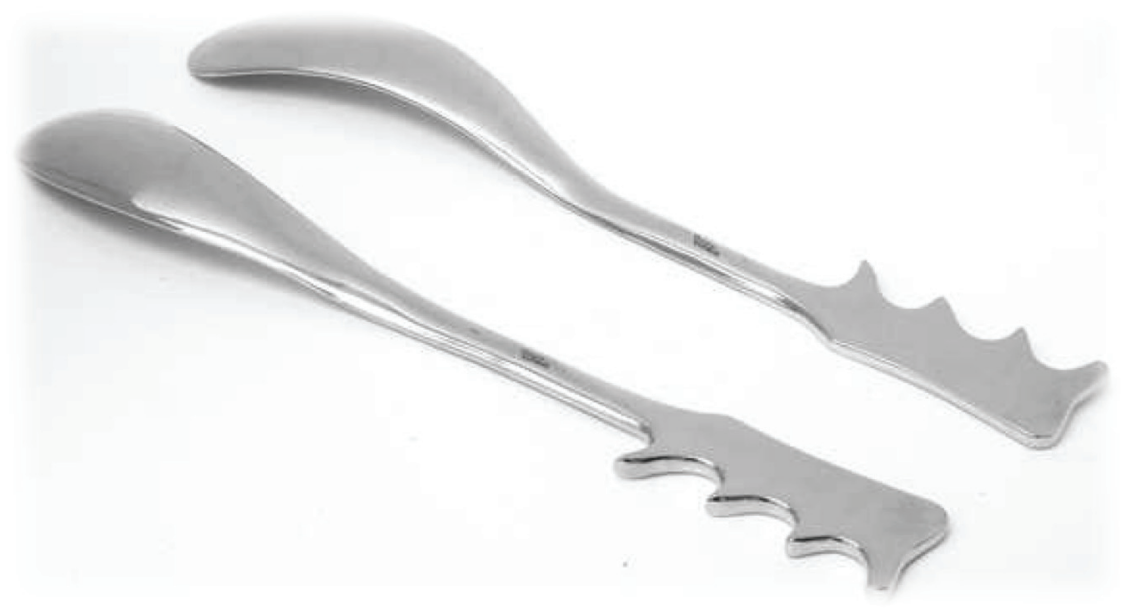

Figure 2. Evolution curve of residents' assessment scores by the senior doctor depending on the resident's curriculum.

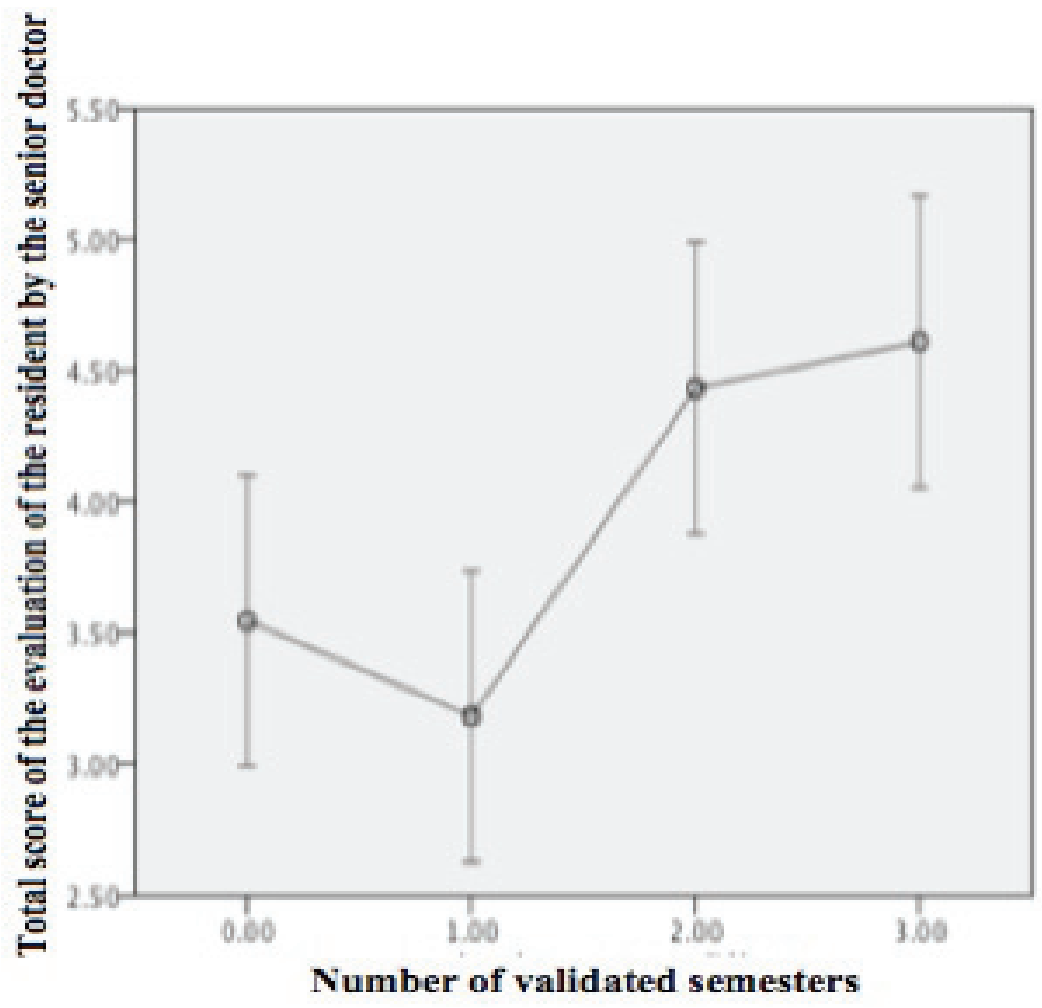


Figure 3. Evaluation by the senior doctor of the resident's competence, based on their seniority/curriculum.

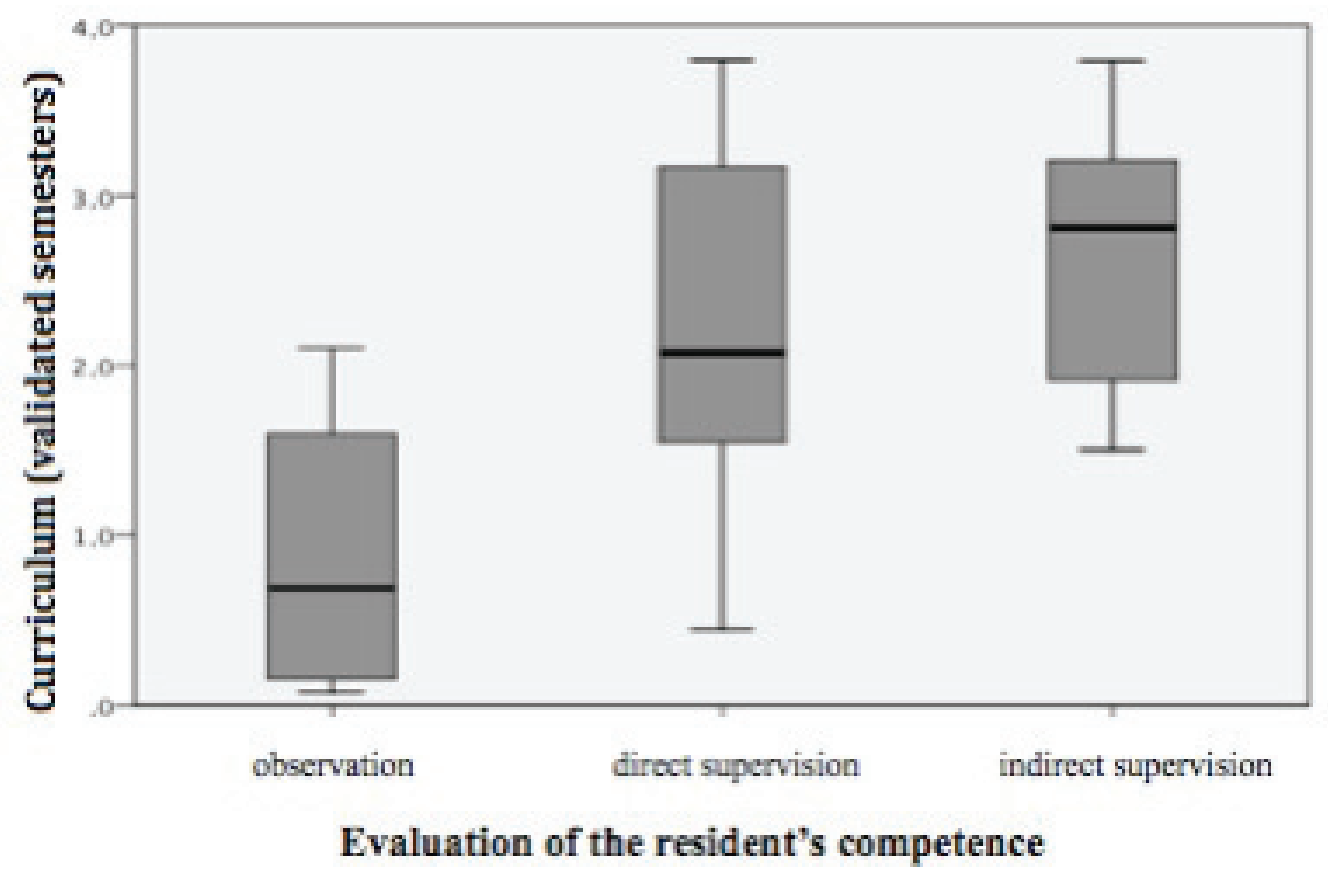

Figure 4. Distribution of the residents' assessment scores by the senior depending on the 20 spatulas extractions threshold embodiment.

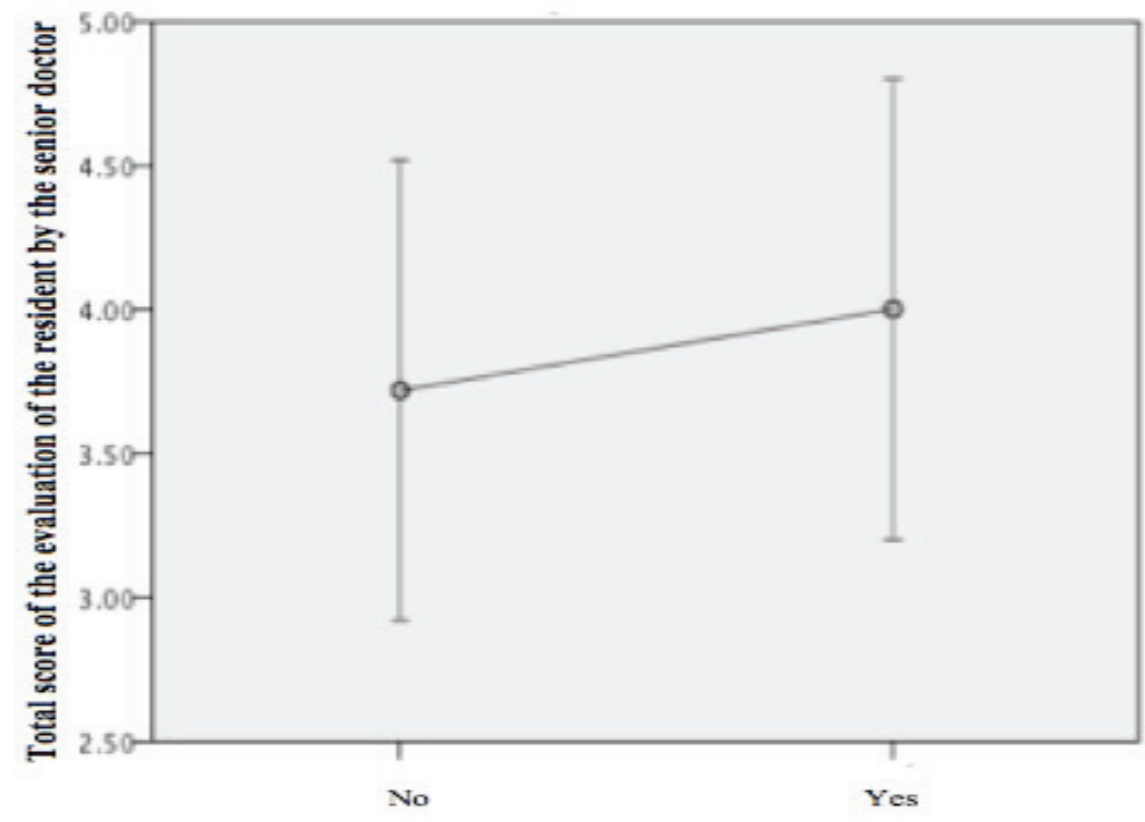

More than 20 spatula-assisted deliveries performed 
Figure 5. Distribution of the confidence level of the resident depending on his curriculum.

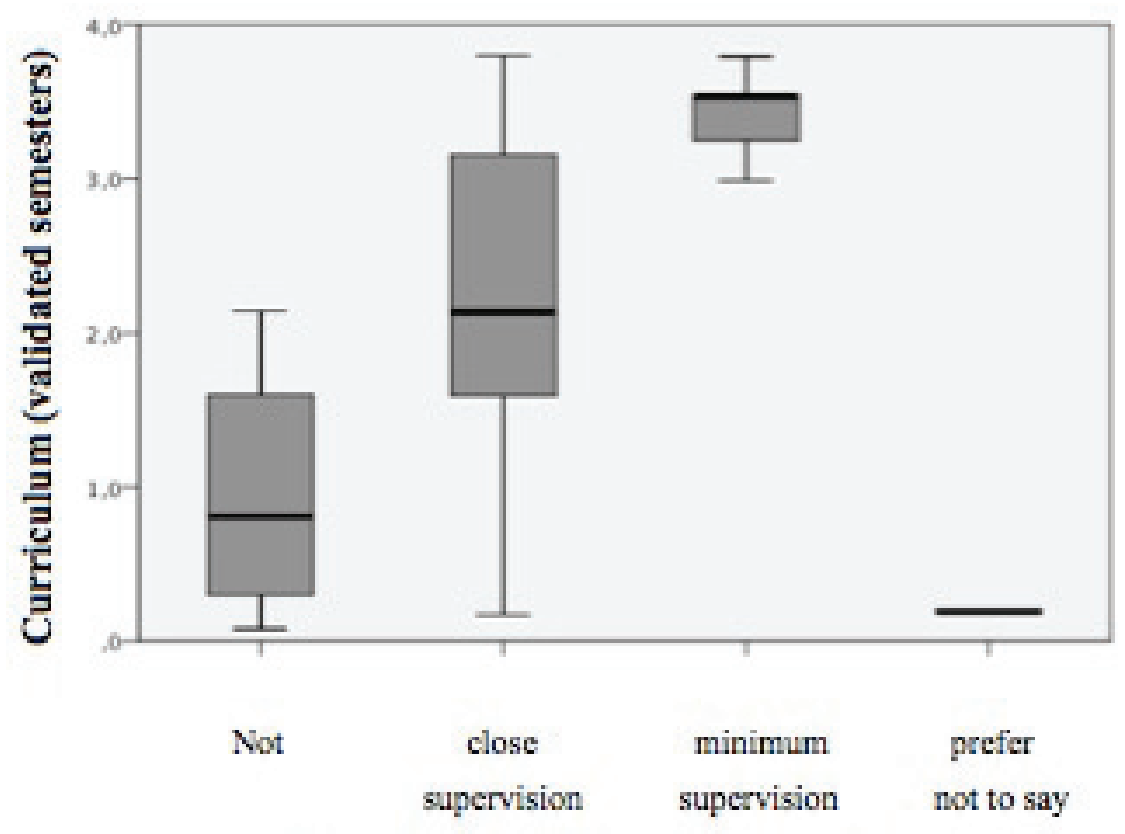

Level of confidence of the resident 
Tables

Table I. Evaluation of the resident's competence: items

\begin{tabular}{|c|c|}
\hline Evaluated item & Mean, Standard deviation, range \\
\hline Analgesia & $4.3+/-0.8(1-5)$ \\
\hline Complete dilatation & $4.6+/-0.8(1-5)$ \\
\hline Asepsis & $4.5+/-0.8(1-5)$ \\
\hline Evaluation of the fetal head engagement & $4+/-0.9(1-5)$ \\
\hline Evaluation of presentation & $3.5+/-1(1-5)$ \\
\hline Urinary catheter drainage & $4.8+/-0.4(3-5)$ \\
\hline Information of the patient & $3.4+/-1(1-5)$ \\
\hline Implementation of the $1^{\text {st }}$ spatula & $3.6+/-1(1-5)$ \\
\hline Implementation of the $2^{\text {nd }}$ spatula & $3.4+/-1(1-5)$ \\
\hline Protection of the soft tissues at implementation & $3.6+/-1(1-5)$ \\
\hline Extraction coordinated with expulsive efforts & $3.5+/-1(2-5)$ \\
\hline Proper tension axis & $3.3+/-1(1-5)$ \\
\hline Propulsion & $3.3+/-1(1-5)$ \\
\hline Evaluation of "asynclitism" & $3.1+/-0.9(1-5)$ \\
\hline Head expulsion gesture & $3.6+/-0.9(1-5)$ \\
\hline Control of the posterior perineum & $3.5+/-1(1-5)$ \\
\hline Body expulsion gesture & $3.7+/-1(2-5)$ \\
\hline Removing spatulas & $3.5+/-1.1(1-5)$ \\
\hline Episiotomy & $3.2+/-1.1(1-5)$ \\
\hline Delivery & $4.4+/-0.8(1-5)$ \\
\hline Tonic uterus & $4.3+/-0.8(1-5)$ \\
\hline Evaluation of perineal lesions & $4.3+/-0.8(1-5)$ \\
\hline Blood loss & $4.1+/-0.9(1-5)$ \\
\hline Interaction with staff & $3.9+/-1.1(1-5)$ \\
\hline
\end{tabular}




\section{Appendices}

Appendix 1: Evaluation form to be completed by the senior doctor

\begin{tabular}{|c|c|c|}
\hline & $\begin{array}{c}\text { scale } 1 \text { to } 5 \\
(5=\text { excellent })\end{array}$ & Non evaluated \\
\hline \multicolumn{3}{|l|}{ Preparation } \\
\hline Analgesia & $1----2----3----4----5$ & \\
\hline Complete dilatation & $1----2----3----4----5$ & \\
\hline Asepsis & $1----2----3----4----5$ & \\
\hline Evaluation of the fetal head engagement & $1----2----3----4----5$ & \\
\hline Evaluation of the type of presentation & $1----2----3----4----5$ & \\
\hline Urinary catheter drainage & $1----2----3----4----5$ & \\
\hline Information of the patient & $1----2----3----4----5$ & \\
\hline \multicolumn{3}{|l|}{ Implementation of spatulas } \\
\hline Implementation of the 1 st spatula & $1----2----3----4----5$ & \\
\hline Implementation of the $2^{\text {nd }}$ spatula & $1----2----3----4----5$ & \\
\hline Protection of the soft tissues & $1----2----3----4----5$ & \\
\hline \multicolumn{3}{|l|}{ Extraction } \\
\hline $\begin{array}{l}\text { Coordination with expulsive efforts and } \\
\text { patient }\end{array}$ & 1----2----3----4----5 & \\
\hline Proper tension axis & 1----2----3----4----5 & \\
\hline Propulsion & $1----2----3----4----5$ & \\
\hline Movements of « asynclitism » & $1----2----3----4----5$ & \\
\hline Head expulsion gesture & $1----2----3----4----5$ & \\
\hline Control of posterior perineum & $1----2----3----4----5$ & \\
\hline Body expulsion gesture & $1----2----3----4----5$ & \\
\hline Removing spatulas & $1----2----3----4----5$ & \\
\hline Episiotomy (moment-size-location) & $1----2----3----4----5$ & \\
\hline Placenta and membranes delivery & $1----2----3----4----5$ & \\
\hline Checking the uterine tone & 1----2----3----4----5 & \\
\hline Evaluation of perineal lesions & $1----2----3----4----5$ & \\
\hline Estimation of blood loss & $1----2----3----4----5$ & \\
\hline Interaction with the rest of the nursing staff & $1----2----3----4----5$ & \\
\hline Writing the report & 1----2----3----4----5 & \\
\hline \multicolumn{3}{|c|}{ Considering the level of difficulty, I think the resident is able to: (check) } \\
\hline \multicolumn{3}{|l|}{ - observe/attend the procedure } \\
\hline \multicolumn{2}{|l|}{ - achieve the procedure with direct supervision } & \\
\hline \multicolumn{2}{|l|}{ - achieve the procedure with indirect supervision } & \\
\hline \multicolumn{2}{|l|}{ - achieve the procedure without supervision } & \\
\hline \multicolumn{2}{|l|}{ - teach the procedure } & \\
\hline - prefer not to say & & \\
\hline
\end{tabular}

Commentaries? Removal of the spatulas at the end of phase 2 of labor? $Y / N$ 
Appendix 2: Evaluation form to be completed by the resident

\begin{tabular}{|c|l|l|}
\hline Interaction Senior/Resident $($ check) & YES & NO \\
\hline Senior present & & \\
\hline Verbal assistance by the senior & & \\
\hline Manual assistance by the senior & & \\
\hline
\end{tabular}

If the senior doctor should help you, the adjustment of the gesture took place during:

\begin{tabular}{|l|l|l|l|}
\hline $\begin{array}{l}\text { Implementation of } \\
\text { spatulas }\end{array}$ & Episiotomy & \\
\hline Traction/Propulsion & $\begin{array}{l}\text { Protection of the } \\
\text { perineum }\end{array}$ & \\
\hline Spatulas removal & Expulsion & \\
\hline Other: (precise) & \multicolumn{2}{|l}{} \\
\hline
\end{tabular}

Circle you answer

1. This case allowed you to improve your acquisition of extractions with spatulas.

g. Strongly agree

h. Okay

i. Neutral

j. Disagree

k. Not agree at all

1. Prefer not to say

2. How do you rate the difficulty of this extraction?
$g$. Very easy
h. Easy
i. Middle
j. Difficult
k. Very difficult
1. Prefer not to say

3. What is your level of confidence extractions with spatulas?
g. No confidence at all
h. Necessity for close supervision
i. Necessity for minimum supervision
j. Can achieve extractions on his own with assistance if needed
$\mathrm{k}$. Can achieve extractions on his own
1. Prefer not to say

Commentaries? $\quad$ Neonatal lesions? Y/N $\quad$ Removal of spatulas at the end of phase 2 of labor? Y/N 


\section{VIII - REFERENCES}

\section{A - FIGURES}

Figure 1 : Données statistiques du mode d'accouchement des mères entre 1994 et 2008 (Association des Utilisateurs de Dossiers Informatisés en Pédiatrie, Obstétrique et Gynécologie. La santé périnatale en France : Tableaux statistiques.

http://www.audipog.net/tablostat.php) 8

Figure 2: Représentation schématique du diaphragme pelvien (vue inférieure) (Kamina P Anatomie clinique de l'appareil génital féminin (Encyclopédie Médico-chirurgical (Elsevier, Paris), Gynécologie, 10-A-10: 1993; 1-28.). .15

Figure 3: Vue d'ensemble des muscles du périnée (Kamina P Anatomie clinique de l'appareil génital féminin (Encyclopédie Médico-chirurgical (Elsevier, Paris), Gynécologie,

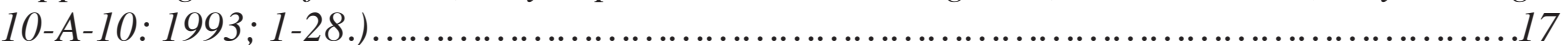

Figure 4: Ampliation du périnée au cours de l'accouchement (Parant $O$, Reme JM, Monrozies X. Déchirures obstétricales récentes du périnée et épisiotomie. EMC ((Elsevier Masson SAS. Paris) - Obstétrique. 1999:1-9 [Article 5-078-A-10])...........................18

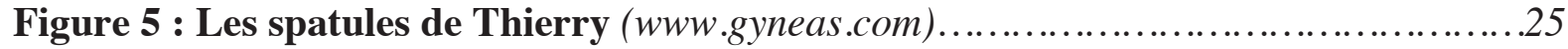

Figure 6 : Principes d'action des spatules (Parant $O$, Simon-Toulza $C$, Fournié A. (Spatules de Thierry. EMC (Elsevier Masson SAS. Paris) - Obstétrique 2011:1-7 [Article 5-095-A-10]).

Figure 7 : Spatules en place selon l'axe sous-occipito-mentonnier (Parant $O$, SimonToulza C, Fournié A (Spatules de Thierry. EMC (Elsevier Masson SAS. Paris) - Obstétrique 2011:1-7 [Article 5-095-A-10]).

Figure 8 : L'effet levier des spatules (Parant $O$, Simon-Toulza $C$, Fournié A Spatules de Thierry. EMC (Elsevier Masson SAS. Paris) - Obstétrique 2011:1-7 [Article 5-095-A-10])..29 
Figure 9: Modification de l'axe des spatules lors de la progression de la présentation (Parant O, Simon-Toulza C, Fournié A. Spatules de Thierry. EMC (Elsevier Masson SAS. Paris) - Obstétrique 2011:1-7 [Article 5-095-A-10]). .29

Figure 10 : La ventouse Kiwi ${ }^{\circledR} .($ mvb-med.com $)$ . .33

Figure 11 : Zone d'action de la ventouse obstétricale sur le cuir chevelu (Schaal JP, Riethmuller D, Menget A. Ventouse obstétricale. EMC (Elsevier Masson SAS. Paris) Obstétrique 2004:1-16 [Article 5-095-A-30]) .............................................34

Figure 12 : Traction dans l'axe ombilicococcygien (Schaal JP, Riethmuller D, Menget A. Ventouse obstétricale. EMC (Elsevier Masson SAS. Paris) - Obstétrique 2004:1-16 [Article

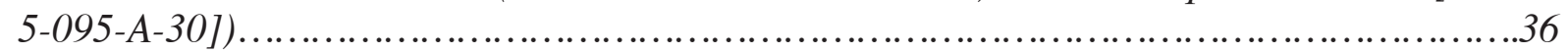

Figure 13 : Forceps de Tarnier (www.gyneas.com) .38

Figure 14 : Un nouvel instrument d'extraction : l'Odon Device (www.odondevice.org)...41

Figure 15 : Répartition géographique régionale des taux d'extractions instrumentales en CHU de niveau III en 2007 (Mangin M, Ramanah R, Aouar Z, Courtois L, Collin A, Cossa $S, \ldots$ \& Riethmuller D. Données 2007 de l'extraction instrumentale en France: résultats d'une enquête nationale auprès de l'ensemble des centres hospitalo-universitaires. Journal de Gynécologie Obstétrique et Biologie de la Reproduction, 2010 ; 39(2), 121132.). . .43

Figure 16 : Distribution géographique régionale de la part des spatules dans l'extraction instrumentale en CHU de niveau III en 2007 (Mangin M, Ramanah R, Aouar Z, Courtois L, Collin A, Cossa S, ... \& Riethmuller D Données 2007 de l'extraction instrumentale en France: résultats d'une enquête nationale auprès de l'ensemble des centres hospitalouniversitaires. Journal de Gynécologie Obstétrique et Biologie de la Reproduction, 2010 ; 39(2), 121-132.). .44

Figure 17 : Distribution géographique régionale de la part des forceps dans l'extraction instrumentale en CHU de niveau III en 2007 (Mangin M, Ramanah R, Aouar Z, Courtois L, Collin A, Cossa S, ... \& Riethmuller D. Données 2007 de l'extraction instrumentale en France: résultats d'une enquête nationale auprès de l'ensemble des centres hospitalouniversitaires. Journal de Gynécologie Obstétrique et Biologie de la Reproduction, 2010 ; 39(2), 121-132.). .45 
Figure 18: Distribution géographique régionale de la part des ventouses dans l'extraction instrumentale en CHU de niveau III en 2007 (Mangin M, Ramanah R, Aouar Z, Courtois L, Collin A, Cossa S, ... \& Riethmuller D. Données 2007 de l'extraction instrumentale en France: résultats d'une enquête nationale auprès de l'ensemble des centres hospitalo-universitaires. Journal de Gynécologie Obstétrique et Biologie de la Reproduction,

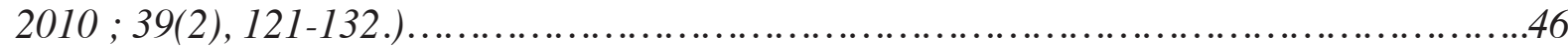

Figure 19 : Diagramme de sélection des extractions par spatules évaluées (Résultats de la $1^{\text {ère }}$ phase notre étude).

Figure 20: Nombre d'extractions évaluées en fonction de l'ancienneté des internes (Résultats de la $1^{\text {ère }}$ phase de notre étude).

Figure 21 : Évaluation par l'obstétricien de garde du niveau de compétence globale de l'interne pour la réalisation des accouchements vaginaux assistés par spatules de Thierry (Résultats de la $1^{\text {ère }}$ phase de notre étude)..............................................59

Figure 22 : Taux de lésion périnéale sévère en fonction du nombre de semestres validés en gynécologie obstétrique (Résultats de la $1^{\text {ère }}$ phase notre étude)

Figure 23 : Evolution des scores totaux des internes évalués par les obstétriciens de garde en fonction de l'ancienneté des internes (Résultats de la $2^{\text {ème }}$ phase notre étude).....67

Figure 24 : Niveau de compétence globale de l'interne selon l'obstétricien de garde (Résultats de la $2^{\text {ème }}$ phase notre étude). . .68

Figure 25: Répartition du niveau de confiance de l'interne en fonction de son ancienneté (Résultats de la $2^{\text {ème }}$ phase notre étude).

Figure 26 : Répartition des scores de l'évaluation des internes par le senior en fonction du seuil de réalisation des 20 extractions par spatules (Résultats de la $2^{\text {ème }}$ phase notre étude). .70

Figure 27 : Schéma théorique des courbes d'apprentissage (Chweitzer A. (1931). VII. Etude expérimentale de la courbe d'apprentissage. L'année psychologique,32(1), 164-196).78 
Figure 28 : Courbes d'apprentissage théorique de l'anesthésie locorégionale en fonction du degré de formation préalable et des moyens utiles à ce stade (Cuvillon $P$, Nouvellon $E$, Ripart J.Apprentissage des techniques d'anesthésie locorégionale. Congrès national d'anesthésie et de réanimation 2007. Évaluation et traitement de la douleur, p. 777-787.)...79

Figure 29: Variation de la vitesse d'apprentissage après mise en situation sur simulateurs (http://cerig.efpg.inpg.fr) ..................................................83

Figure 30 : The BirthSIM Simulator (Dupuis O, Decullier E, Clerc J, et al. Training in forceps blade placement: Birth Simulator training allows Obstetrician's skills improvment 2008.).....

Figure 31 : Description du modèle d'apprentissage : les étapes du modèle de Butler (Thouin A, Creveuil C. L'apprentissage de la médecine à la lumière du modèle de Butler. Pédagogie médicale.2001 ; 2(4), 222-230.)

Figure 32 : Laerdal : console de paramétrage SimMom ${ }^{\circledR}$ (Bouin, T. (2013). La simulation sur mannequin haute fidélité dans la formation initiale des sages-femmes. La Revue SageFemme.). 


\section{B - BIBLIOGRAPHIE}

[1] Maisonnette-Escot Y, Riethmuller D, Chevrière S, Becher P, Floret N, Zurlinden B et al. L'extraction instrumentale par spatules de Thierry : étude de la morbidité maternofœtale .Gynecologie Obstetrique \& Fertilite. 2005 ; 33 : 208-212.

[2] De Troyer J, Bouvenot J, D’Ercole C, Boubli L. Extraction instrumentale par spatules de Thierry : à propos de 166 cas. Journal de gynécologie, obstétrique et biologie de la reproduction. $2005 ; 34: 795-801$.

[3] Boucoiran I, Valerio L, Bafghi A, Delotte J, \& Bongain A. Spatula-assisted deliveries: a large cohort of 1065 cases. European journal of obstetrics, gynecology, and reproductive biology. $2010 ; 151(1), 46-51$.

[4] Baud O. Complications néonatales des extractions instrumentales. Journal de Gynécologie Obstétrique et Biologie de la Reproduction.2008 ; 37(8), S260-S268.

[5] Schaal JP, Riethmuller D, \& Berthet J. Manœuvres instrumentales et manuelles lors de l'accouchement: Accouchement. La Revue du praticien.1999 ; 49(2), 139-145.

[6] Berthet J. Forceps : cur, quando, comodo (pourqoi, quand, comment). Encyclopédie Médico-chirurgical, Elsevier, Paris, Obstétrique.2003 ; 5-095-A-20, 8 p.

[7] Aouar Z, Ramanah R, Mangin M, Leung F, Mulin B, Courtois L, et al. Morbidité materno-fœtale liée à l'extraction instrumentale par spatules de Teissier. Étude comparative à la ventouse obstétricale de type Minicup ${ }^{\circledR}$ Journal de Gynécologie Obstétrique et Biologie de la Reproduction.2009; 38 : 642-647.

[8] Mazouni C, Bretelle F, Collette E, Heckenroth H, Bonnier P, Gamerre M. Maternal and neonatal morbidity after first vaginal delivery using Thierry's spatulas.Journal Australian and New Zealand Journal of Obstetrics and Gynaecology .2005; 45 : 405-409.

[9] Menard JP, Provansal M, Heckenroth H, Gamerre M, Bretelle F, Mazouni C. Morbidité maternelle immédiate après extraction instrumentale par spatules de Thierry et par ventouse obstétricale Gynecologie Obstetrique \& Fertilite .2008; 36 : 623-627.

[10] Parant O, Simon-Toulza C, Capdet J, Fuzier V, Arnaud C, Rème JM. Morbidité maternofœtale immédiate de l'accouchement assisté par spatules de Thierry chez la primipare. À 
propos d'une série prospective continue de 195 extractions. Gynecologie Obstetrique \& Fertilite.2009; 37 : 780-786.

[11] Vanlieferinghen S, Girard G, Mandelbrot L. Étude comparative de la morbidité maternofœetale immédiate des extractions par spatules de Thierry et par ventouses.Journal de gynécologie, obstétrique et biologie de la reproduction. $2009 ; 38$ : 648-654.

[12] Boucoiran I, Bafghi A, Delotte J, Valerio L, Bongain A. Risques néonataux et périnéaux liés à l'apprentissage des spatules. Journal de gynécologie, obstétrique et biologie de la reproduction.2010; $39: 224-230$.

[13] Courtois L, Becher P, Maticot-Baptista D, Cour A, Zurlinden B, Millet P, et al. Extraction instrumentale par spatules de Thierry : évaluation du risque périnéal en fonction du dégagement. Journal de gynécologie, obstétrique et biologie de la reproduction.2008 ; 37 : 276-282.

[14] Parant O, Simon C, Capdet J, Tanguy Le Gac Y, Reme JM. Peut-on encore réaliser des rotations instrumentales par spatules de Thierry ? Étude préliminaire chez la primipare. Journal de gynécologie, obstétrique et biologie de la reproduction 2007 ; 36 : 582-587.

[15] European Board and College of Obstetricians and Gynaecologists. Training Assessment. Edit 18/03/2008.http://www.cngof.asso.fr/D_TELE/eu_logb.pdf

[16] CNGOF. Recommandations pour la pratique clinique : extractions instrumentales (2008). Journal de Gynécologie Obstétrique et Biologie de la Reproduction .2008; 37:S173-299.

[17] Lloyd FP, Geisler HE, \& Lushbaugh H. Unarticulated forceps in opérative obstetrics.The spatulas of Thierry.American journal of obstetrics and gynecology.1964. 90, 925.

[18] Berthet J,Racinet D. Extractions instrumentales du foetus. EMC (Elsevier Masson SAS. Paris) - Obstétrique 1993:1-0 [Article 5-095-A-10].

[19] Soutoul JH, Bertrand J, Pierre F. Le gynécologue obstétricien face au juge. Institut Schering. Bruxelles. $1989 ; 222$.

[20] Vokaer R, De Grandi P, Giraud J, Hohlfeld P, Moutquin J, Pierre F, ... \& Vokaer A. Médicalisation ou non de l'accouchement. Journal de gynécologie obstétrique et biologie de la reproduction.1990. 19(5), 607-618. 
[21] Dierker Jr LJ, Rosen MG, Thompson K, \& Lynn P. Midforceps deliveries: long-term outcome of infants. American journal of obstetrics and gynecology.1986.154 (4), 764.

[22] Seidman DS, Laor A, Gale R, Stevenson D K, Mashiach S, \& Danon YL. Long-term effects of vacuum and forceps deliveries. Lancet.1991.337(8757), 1583-1585.

[23] Association des Utilisateurs de Dossiers Informatisés en Pédiatrie, Obstétrique et Gynécologie. La santé périnatale en France : Tableaux statistiques.

http://www.audipog.net/tablostat.php

[24] Sentilhes L, Gillard P, Descamps P, \& Fournié A. Indications et prérequis à la réalisation d'une extraction instrumentale: quand, comment et où? Journal de Gynécologie Obstétrique et Biologie de la Reproduction.2008. 37(8), S188-S201.

[25] American College of Obstetricians and Gynecologists. Operative Vaginal Delivery: use of forceps and vacuum extractors for operative vaginal delivery. ACOG Practice Bulletin No 17, June 2000. Washington DC: ACOG; 2000.

[26] Royal College of Obstetricians and Gynaecologists. Operative Vaginal Delivery. Guideline No 26, revised October 2005. [www.rcog.org.uk].

[27] Dupuis O, Dubuisson J, Moreau R, Sayegh I, Clément HJ \& Rudigoz RC. Rapidité d'extraction respective des césariennes et des forceps réalisés en urgence: À propos de 137 extractions pour anomalies du rythme cardiaque fotal en cours de travail. Journal de gynécologie obstétrique et biologie de la reproduction.2005. 34(8), 789-794.

[28] SOGC Clinical practice guideline No 148, August 2004 Guidelines for operative vaginal birth. Journal of Obstetrics and Gynaecology Canada.2004 ; 26 : 747-753.

[29] Royal Australian and New Zealand College of Obstetricians Gynaecologists. Instrumental Vaginal Delivery. College Statement No C-Obs 16. Melbourne: RANZCOG; 2004.

[30] Durieux P, Roche N, Dosquet P, Pazart L, \& Charvet-Protat S. Les recommandations pour la pratique clinique. L'évaluation médicale, du concept à la pratique. Paris: Flammarion.1994.48-59.

[31]Analyse littérature médicale - Guide méthodologique. Haute autorité de santé.2000. http://www.hassante.fr/portail/upload/docs/application/pdf/analiterat.pdf 
[32] Demissie K, Rhoads GG, Smulian JC, Balasubramanian BA, Gandhi K, Joseph KS, \& Kramer M. Operative vaginal delivery and neonatal and infant adverse outcomes: population based retrospective analysis.British Medical Journal.2004.329 (7456), 24.

[33] De Leeuw JW, De Wit C, Kuijken JP JA, \& Bruinse HW. Mediolateral episiotomy reduces the risk for anal sphincter injury during operative vaginal delivery. BJOG: An International Journal of Obstetrics \& Gynaecology.2008. 115(1), 104-108.

[34] Gardella C, Taylor M, Benedetti T, Hitti J, \& Critchlow C. The effect of sequential use of vacuum and forceps for assisted vaginal delivery on neonatal and maternal outcomes. American journal of obstetrics and gynecology.2001. 185(4), 896.

[35] Eason E, Labrecque M, Marcoux S, \& Mondor M. Anal incontinence after childbirth. Canadian Medical Association Journal.2002. 166(3), 326-330.

[36] Mazouni C., Bretelle F, Battar S, Bonnier P, \& Gamerre M. Frequency of persistent anal symptoms after first instrumental delivery. Diseases of the colon \& rectum.2005. 48(7), 14321436.

[37] Kabiru W N, Jamieson D, Graves W, \& Lindsay M. Trends in operative vaginal delivery rates and associated maternal complication rates in an inner-city hospital. American journal of obstetrics and gynecology.2001. 184(6), 1112-1114.

[38] Rortveit G, Daltveit AK, Hannestad YS, \& Hunskaar S. Urinary incontinence after vaginal delivery or cesarean section. New England Journal of Medicine.2003 . 348(10), 900907.

[39] Chiaffarino F, Chatenoud L, Dindelli M, Meschia M, Buonaguidi A, Amicarelli F, ... \& Parazzini F. Reproductive factors, family history, occupation and risk of urogenital prolapse. European Journal of Obstetrics \& Gynecology and Reproductive Biology.1999.82 (1), 63-67.

[40] Macarthur AJ, \& Macarthur C. Incidence, severity, and determinants of perineal pain after vaginal delivery: a prospective cohort study. American Journal of Obstetrics and Gynecology.2004.191 (4), 1199-1204.

[41] Williams A, Herron-Marx S, \& Carolyn H. The prevalence of enduring postnatal perineal morbidity and its relationship to perineal trauma. Midwifery, 2007. 23(4), 392. 
[42] Bahl R, Strachan B, \& Murphy DJ. Outcome of subsequent pregnancy three years after previous operative delivery in the second stage of labour: cohort study. British Medical Journal.2004 328(7435), 311.

[43] Kamina P Anatomie clinique de l'appareil génital féminin. Encyclopédie Médicochirurgical (Elsevier, Paris), Gynécologie, 10-A-10: 1993; 1-28.

[44] Parant O, Reme JM, Monrozies X. Déchirures obstétricales récentes du périnée et épisiotomie. EMC ((Elsevier Masson SAS. Paris) - Obstétrique. 1999:1-9 [Article 5-078-A$10]$.

[45] International classification of diseases. Word Health Organisation. 10th révision (ICD10-O70). Geneva, Switzerland1996.

[46] Beucher G. Complications maternelles des extractions instrumentales. Journal de Gynécologie Obstétrique et Biologie de la Reproduction.2008. 37(8), S244-S259.

[47] Richter HE, Brumfield CG, Cliver SP, Burgio KL, Neely CL, Varner RE. Risk factors associated with anal sphincter tear: a comparison of primiparous patients, vaginal births after cesarean deliveries, and patients with previous vaginal delivery.American Journal of Obstetrics and Gynecology.2002 ; 187 : 1194-1198.

[48] Christianson LM, Bovbjerg VE, McDavitt EC. Hullfish KL. Risk factors for perineal injury during delivery .American Journal of Obstetrics and Gynecology.2003 ; 189 : 255260.

[49] Barbier A, Poujade O, Fay R, Thiébaugeorges O, Levardon M, Deval B. La primiparité est-elle le seul facteur de risque des lésions du sphincter anal en cours d'accouchement.Gynecologie Obstetrique \& Fertilite.2007 ; 35 : 101-106.

[50] Dahlen HG, Ryan M, Homer CSE, Cooke M. An Australian prospective cohort study of risk factors for severe perineal trauma during childbirth. Midwifery .2007; 23 : 196-203.

[51] Lowder JL, Burrows LJ, Krohn MA, Weber AM. Risk factors for primary and subsequent anal sphincter lacerations: a comparison of cohorts by parity and prior mode of delivery.American Journal of Obstetrics and Gynecology.2007 ; 196 : 344. e1-e5. 
[52] De Leeuw JW, Struijk PC, Vierhout ME, Wallenburg HC. Risk factors for third degree perineal ruptures during delivery. BJOG: An International Journal of Obstetrics and Gynaecology.2001; $108: 383-387$.

[53] Handa VL, Danielsen BH, Gilbert WM. Obstetrical anal sphincter lacérations. Obstetrics and Gynecolog.y.2001; $98: 225-230$.

[54] Di Piazza D, Richter HE. Chapman V, Cliver SP, Neely C, Chen CC, et al. Risk for anal sphincter tear in multiparas .Obstetrics and Gynecology.2006 ; 107 : 1233-1237.

[55] Angioli R, Gomez-Marin O, Cantuaria G, O’Sullivan MJ. Severe perineal lacerations during vaginal delivery: the University of Miami Experience American Journal of Obstetrics and Gynecology.2000；182:1083-1085.

[56] Jolly MC, Sebire NJ, Harris JP, Regan L, Robinson S. Risk factors for macrosomia and its clinical consequences: a study of 350311 pregnancies .European journal of obstetrics, gynecology, and reproductive biology. $2003 ; 111: 9-14$.

[57] Fitzpatrick M, McQuillan K, O’Herlihy C. Influence of persistent occiput posterior position on delivery outcome .Obstetrics and Gynecology.2001; 98 : 1027-1031.

[58] Ponkey SE, Cohen AP, Heffner LJ, Lieberman E. Persistent fetal occiput posterior position: obstetric outcomes .Obstetrics and Gynecology.2003 ; 101 : 915-920.

[59] Naime-Alix AF, Fourquet F, Sigue D, Potin J, Descriaud C, Perrotin F. Combien de temps peut-on attendre à dilatation complète ? Analyse de la morbidité maternelle et fotale selon la durée de la seconde phase du travail chez la primipare.Journal de gynécologie, obstétrique et biologie de la reproduction.2008; 37 :268-275.

[60] Langer B, Minetti A. Complications immédiates et à long terme de l'épisiotomie J Gynecol Obstet Biol Reprod 2006 ; 35 (Suppl 1) : 1S59-1S67.

[61] Rodriguez A, Arenas EA, Osorio AL, Mendez O, Zuleta JJ. Selective vs routine midline episiotomy for the prevention of third- or fourth-degree lacerations in nulliparous women. American Journal of Obstetrics and Gynecology.2008 ; 198 : 285. e1-4.

[62] Gupta JK, Nokodem VC. Woman's position during second stage of labour .Cochrane Database of Systematic Reviews.2004 ; CD002006. 
[63] Samuelsson E, Ladfors L, Wennerholm UB, Gareberg B, Nyberg K, Hagberg H. Anal sphincter tears: prospective study of obstetric risk factors.$B J O G$ : An International Journal of Obstetrics and Gynaecology. $2000 ; 107$ : 926-931.

[64] Pirhonen JP, Grenman SE, Haadem K, et al. Frequency of anal sphincter rupture at delivery in Sweden and Finland - Results of difference in manual help to the baby's head Acta Obstetricia et Gynecologica Scandinavica.1998 ; 77 : 74-77.

[65] Riethmuller D, Pequegnot-Jeannin C, Rabenja CA, Koeberle P, Schaal J P, \& Maillet R A rare cause of postpartum hemorrhage: a genital thrombus]. Journal de gynécologie, obstétrique et biologie de la reproduction.1997. 26(2), 154.

[66] Saleem Z, \& Rydhström H. Vaginal hematoma during parturition: a population-based study. Acta Obstetricia et Gynecologica Scandinavica.2004. 83(6), 560-562.

[67] COMBS CA, Murphy E L, \& Laros Jr RK. Factors associated with postpartum hemorrhage with vaginal birth. Obstetrics \& Gynecology.1991. 77(1), 69-76.

[68] Stones RW, Paterson CM, \& StG Saunders N J. Risk factors for major obstetric haemorrhage. European Journal of Obstetrics \& Gynecology and Reproductive Biology.1993. 48(1), 15-18.

[69] Dupuis O, Silveira R, Redarce T, Dittmar A, \& Rudigoz R C.Extraction instrumentale en 2002 au sein du réseau AURORE: incidence et complications néo-natales graves.

Gynécologie obstétrique \& fertilité.2003.31 (11), 920-926.

[70] Thierry, E Nouvel instrument destiné à remplacer le forceps. París: La Presse Medical.1950. 81, 1423-4.

[71] Teissier G, Miliani P, Guidicelli C, Jubelin J. Présentation d'un nouveau type de spatules. Bulletin de la Fédération des Sociétés de Gynécologie et d'Obstétrique de Langue Française .1971; $23: 343-344$.

[72] Parant O, Simon-Toulza C, Fournié A. Spatules de Thierry. EMC (Elsevier Masson SAS. Paris) - Obstétrique 2011:1-7 [Article 5-095-A-10].

[73] Martin-Laval J. À propos des spatules de Thierry. Bulletin de la Fédération des Sociétés de Gynécologie et d'Obstétrique de Langue Française .1956; 8 : 637-638. 
[74] Guyomar J. Valeur obstétricale des spatules de Thierry. Résultats de 1000 applications.Revue française de gynécologie et d'obstétrique.1965; 40 : 775-783.

[75] Grisot C, Mancini J, de Troyer J, Rua S, Boubli L, D’Ercole C, \& Carcopino X. Morbidité périnéale des extractions instrumentales par spatules et ventouses: qu'en est-il réellement? .Journal de Gynécologie Obstétrique et Biologie de la Reproduction.2011. 40(4), 348-358.

[76] Schaal JP, Riethmuller D, Menget A. Ventouse obstétricale. EMC (Elsevier Masson SAS. Paris) - Obstétrique 2004:1-16 [Article 5-095-A-30].

[77] Edozien LC, Williams JL, Chatterjee IC, Hirsch PJ. Failed instrumental delivery: how safe is the use of a second instrument? .Journal of Gynecology \& Obstetrics. 1999; 19:460-2.

[78] Plauche WC. Fetal cranial injuries related to delivery with the Malmstrom vacuum extractor. Obstetrics and Gynecology.1979; 53:750-7.

[79] Berkus MD, Ramamurthy RS, O'Connor PS, Brown K, Hayashi RH. Cohort study of silastic obstetric vacuum cup deliveries: I. Safety of the instrument. Obstetrics and Gynecology. 1985; 66:503-9.

[80] Berthet J. Forceps. EMC EMC (Elsevier Masson SAS. Paris) - Obstétrique 2011:1-13 [Article 5-095-A-20].

[81] Caughey AB, Sandberg PL, Zlatnik MG, Thiet MP, Parer JT, Laros RK. Forceps compared versus vacuum: rates of neonatal and maternal morbidity. Obstetrics and Gynecology.2005 ; 106 : 908-91.

[82] Douvier S. Complications maternelles des forceps Mécanique et techniques obstétricales Montpellier: Sauramps Médical. 665-673.

[83] www.odondevice.org/

[84] Mangin M, Ramanah R, Aouar Z, Courtois L, Collin A, Cossa S, .. \& Riethmuller D. Données 2007 de l'extraction instrumentale en France: résultats d'une enquête nationale auprès de l'ensemble des centres hospitalo-universitaires. Journal de Gynécologie Obstétrique et Biologie de la Reproduction, 2010 ; 39(2), 121-132. 
[85] Dupuis O. Formation et apprentissage des extractions. Journal de Gynécologie Obstétrique et Biologie de la Reproduction.2008. 37(8), S288-S296.

[86] Schaal JP, Rabenja CA, Gay C, Riethmuller D, Maillet R. Extractions instrumentales et césariennes dans les CHU français en 1993 .XXV Assises nationales des sage-femmes .1993.

[87] ACOG Practice Bulletin. Operative vaginal delivery: clinical managements guidelines for obstetrician-gynecologists (2000). International Journal of Gynecology \& Obstetrics.2001; 74:69-76.

[88] Bergsjo P. Differences in the reported frequencies of some obtetrical interventions in Europe .BJOG: An International Journal of Obstetrics and Gynaecology.1983 ; 90 : 628-632.

[89] McLeod M, Murphy DJ. Operative vaginal delivery and the use of episiotomy - A survey of practice in the United Kingdom and Ireland. European journal of obstetrics, gynecology, and reproductive biology. $2008 ; 136: 178-183$.

[90] Sau A, Sau M, Ahmed H, Brown R. Vacuum extraction: is there any need to improve the current training in the UK? Acta Obstetricia et Gynecologica Scandinavica.2004 ; 83 : 466470 .

[100] Johanson R, Menon B. Vacuum extraction versus forceps for assisted vaginal delivery. Cochrane Database of Systematic Reviews.2000; 2:CD000224.

[101] Powell J, Gilo N, Foote M, Gil K, Lavin JP. Vacuum and forceps training in residency: experience and self-reported competency. Journal of Perinatology.2007 ; 27 : 343-346.

[102] Achanna S, Monga D. Outcome of forceps delivery versus vacuum extraction--a review of 200 cases Singapore Medical Journal .1994; 35 : 605-608.

[103] Driscoll IB. Relative safety of vacuum extraction vs Kielland forceps

delivery.Australian and New Zealand Journal of Obstetrics and Gynaecology .2000; 40 : 226.

[104] Jude DC, Gilbert GG, \& Magrane D. Simulation training in the obstetrics and gynecology clerkship. American journal of obstetrics and gynecology.2006. 195(5), 14891492.

[105] Fermanian J. Validation des échelles d'évaluation en médecine physique et de réadaptation: comment apprécier correctement leurs qualités psychométriques. In Annales de réadaptation et de médecine physique. Elsevier Masson.2005. Vol. 48, No. 6, pp. 281-287. 
[106] Martin-Laval J, Deturmeny G, Zographos G. Notre expérience actuelle sur l'application des spatules de Thierry .Revue française de gynécologie et d'obstétrique. 1958 ; 53 : 517 524.

[107] Fournié A, Parant O. Technique d'application des spatules de Thierry Techniques chirurgicales et obstétricales : Collège national des gynécologues et obstétriciens français; Cassette VIII (1999).

[108] Edozien LC. Towards safe practice in instrumental vaginal delivery. Best Pract Res Clin Obstet Gynaecol 2007; 21: 637-55.

[109] Chweitzer A. (1931). VII. Etude expérimentale de la courbe d'apprentissage. L'année psychologique, 32(1), 164-196.

[110] Cuvillon P, Nouvellon E, Ripart J.Apprentissage des techniques d'anesthésie locorégionale. Congrès national d'anesthésie et de réanimation 2007. Évaluation et traitement de la douleur, p. 777-787.

[111] Dupuis O, Decullier E, Clerc J, et al. Training in forceps blade placement: Birth Simulator training allows Obstetrician's skills improvment 2008.

[112] Velemir L, Vendittelli F, Bonnefoy C, Accoceberry M, Savary D, \& Gallot, D. Courbe d'apprentissage de la ventouse obstétricale par les internes: étude préliminaire. Journal de Gynécologie Obstétrique et Biologie de la Reproduction.2009 ; 38(5), 421-429.

[113] Ennen, CS, \& Satin AJ. Training and assessment in obstetrics: the role of simulation. Best Practice \& Research Clinical Obstetrics \& Gynaecology.2010 ; 24(6), 747-758.

[114] http://www.agof.info/PDF/livretdelinterne 2008.pdf

[115] Rathat G, Hoa D, Gagnayre R, Hoffet M, \& Mares P.Formation chirurgicale des internes, spécialistes en gynécologie-obstétrique: résultats d'une enquête électronique nationale. Journal de Gynécologie Obstétrique et Biologie de la Reproduction.2008 ; 37(7), 672-684.

[116] Martin JA, Regehr G, Reznick R, MacRae H, Murnaghan J, Hutchison C, \& Brown, M. Objective structured assessment of technical skill (OSATS) for surgical residents. British Journal of Surgery.1997; 84(2), 273-278. 
[117] Swift SE, \& Carter JF. Institution and validation of an observed structured assessment of technical skills (OSATS) for obstetrics and gynecology residents and faculty. American journal of obstetrics and gynecology.2006; 195(2), 617-621.

[118] Goff BA, Lentz GM, Lee D, Houmard B, \& Mandel LS .Development of an objective structured assessment of technical skills for obstetric and gynecology residents. Obstetrics \& Gynecology.2000. 96(1), 146-150.

[119] MOREAU R. Le simulateur d'accouchement BirthSIM: un outil complet pour la formation sans risque en obstétrique. Thèse de doctorat. INSA de Lyon.2007.

[120] Thouin A, Creveuil C. L'apprentissage de la médecine à la lumière du modèle de Butler. Pédagogie médicale.2001;2(4), 222-230.

[121] Bouin, T. (2013). La simulation sur mannequin haute fidélité dans la formation initiale des sages-femmes. La Revue Sage-Femme.

[122] Dijksterhuis M G, Voorhuis M, Teunissen PW, Schuwirth LW, Ten Cate OT, Braat DD, \& Scheele F.Assessment of competence and progressive independence in postgraduate clinical training. Medical éducation.2009 ; 43(12), 1156-1165.

[123] Glenn Posner MDCM,Amy Nakajmia MD. Evaluation of Residents' Documentation Skills After a Simulated Operative Vaginal Delivery. Journal of obstetrics and gynaecology Canada.2009 ; 31(11), 1064-1067.

[124] http://www.cngof.asso.fr/D TELE/eu logb.pdf

[125] Lane S, Weeks A, Scholefield H, Alfirevic Z. Monitoring obstetricians' performance with statistical process control charts. BJOG: An International Journal of Obstetrics and Gynaecology.2007; 114:614-618.

[126] Bendifallah S. The forthcoming reforms of residentship and Post- residentship in obstetrics and gynaecology: why and how? Gynecologie Obstetrique \& Fertilite.2012; 40:623.

[127] Bendifallah S. Course of study, the end of the basic surgery training for residents in obstetrics and gynaecology. Gynecologie Obstetrique \& Fertilite.2011; 39:265. 
[128] Chanavaz-Lacheray I, Nizard J. Démographie de la gynécologie- obstétrique en France en 2003. Journal de gynécologie, obstétrique et biologie de la reproduction.2005; 34:321-7.

[129] Granry JC, Moll MC. Rapport de mission: État de l'art (national et international) en matière de pratiques de simulation dans le domaine de la santé. Haute Autorité de santé. 2012. Rapport accessible sur http://www. has-sante. fr/portail/upload/docs/application/pdf/201201/simulation_en_sante_-_rapport.pdf

[130] Bréaud J, Chevallier D, Benizri E, Fournier JP, Carles M, Delotte J, ... \& Benchimol D. Intégration de la simulation dans la formation des internes en chirurgie. Programme pédagogique du centre de simulation médicale de la faculté de médecine de Nice. Journal de Chirurgie Viscérale.2012;149(1), 55-63.

[131] Gardner R,Raemer DB. Simulation in obstetrics and gynecology. Obstetrics and Gynecology Clinics.2008; 35(1).

[132] Tassin M, Cordier AG, Laher G, Benachi , \& Mandelbrot L. Simulateur d'amniocentèse: intérêts et développement d'un nouveau modèle reproductible et économique. Journal de Gynécologie Obstétrique et Biologie de la Reproduction.2012 ; 41(7), 679-683. 


\section{SERMENT D'HIPPOCRATE}

«En présence des Maîtres de cette école et de mes chers condisciples et devant l'effigie d'Hippocrate,

Je promets et je jure d'être fidèle aux lois de l'honneur et de la probité dans l'exercice de la médecine.

Je donnerai mes soins gratuits à l'indigent et n'exigerai jamais un salaire au dessus de mon travail.

Admis à l'intérieur des maisons, mes yeux ne verront pas ce qu'il s'y passe, ma langue taira les secrets qui me seront confiés et mon état ne servira pas à corrompre les mœurs ni à favoriser le crime.

Je ne permettrai pas que des considérations de religion, de nation, de race, de parti ou de classe sociale viennent s'interposer entre mon devoir et mon patient.

Je garderai le respect absolu de la vie humaine.

Même sous la menace, je n'admettrai pas de faire usage de mes connaissances médicales contre les lois de l'humanité.

Respectueux et reconnaissant envers mes maîtres, je donnerai à leurs enfants l'instruction que j'ai reçue de leur père.

Que les Hommes m'accordent leur estime si je suis fidèle à mes promesses. Que je sois couvert d'opprobre et méprisé de mes confrères si j'y manque. » 